IS-T -536

\title{
CONTRIBUTIONS OF TIME-REVERSAL NONINVARIANT \\ TERMS IN ELECTRON-NUCLEUS ELASTIC \\ SCATTERING
}
Ph. D. Thesis Submitted to Iowa State University, August, 1972

L. D. Krase

Ames Laboratory, USAEC

Iowa State University

Ames, Iowa 50010

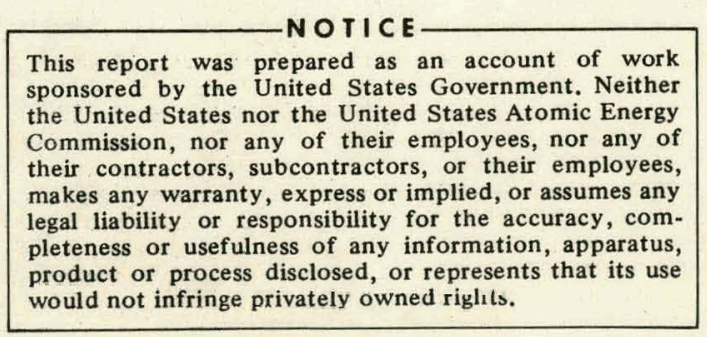

Date of Manuscript: August, 1972

PREPARED FOR THE U. S. ATOMIC ENERGY COMMISSION DIVISION OF RESEARCH UNDER CONTRACT W-7405-eng-82 


\section{DISCLAIMER}

This report was prepared as an account of work sponsored by an agency of the United States Government. Neither the United States Government nor any agency Thereof, nor any of their employees, makes any warranty, express or implied, or assumes any legal liability or responsibility for the accuracy, completeness, or usefulness of any information, apparatus, product, or process disclosed, or represents that its use would not infringe privately owned rights. Reference herein to any specific commercial product, process, or service by trade name, trademark, manufacturer, or otherwise does not necessarily constitute or imply its endorsement, recommendation, or favoring by the United States Government or any agency thereof. The views and opinions of authors expressed herein do not necessarily state or reflect those of the United States Government or any agency thereof. 


\section{DISCLAIMER}

Portions of this document may be illegible in electronic image products. Images are produced from the best available original document. 


This report was prepared as an account of work
sponsored by the United States Government. Neither
the United States nor the United States Atomic
Energy Commission, nor any of their employees, nor
any of their contractors, subcontractors, or their
employees, makes any warranty, express or implied,
or assumes any legal liability or responsibility for the
accuracy, completeness or usefulness of any
information, apparatus, product or process disclosed,
or represents that its use would not infringe privately
owned rights.

Available from: National Technical Information Service Department $A$

Springfield, VA 22151

Price: Microfiche $\$ 0.95$ 


\section{Contributions of time-reversal noninvariant terms \\ in electron-nucleus elastic scattering \\ by

\author{
Loren Dell Krase \\ A Dissertation Submitted to the \\ Graduate Faculty in Partial Fulfillment of \\ DOCTOR OF PHILOSOPHY
} \\ The Requirements for the Degree of}

Department: Physics

Major: Elementary Particle Physics

Approved:

$\frac{D 2}{\text { In Charge of Major Work }} 72$

$\frac{02.6: 03 a m e s}{\text { For the Major Department }}$

$\overline{\text { For the Graduate College }}$

lowa State University

of Science and Technology

Ames, lowa

1972 


\section{ABSTRACT}

I. INTRODUCTION

11. ONE PHOTON EXCHANGE APPROXIMATION OF ELASTIC ELECTRON-NUCLEUS SCATTERING

A. Elements of Scattering Theory

B. Construction of the Current in the $(0, s) \oplus(s, 0)$ Representation

C. The "Boosted" Current

111. HIGHER ORDER APPROXIMATIONS OF THE ELASTIC SCATTERING PROCESS

IV. DISCUSSION OF SOME OF THE OBSERVABLES

IN THE ELASTIC SCATTERING PROCESS

A. Unoriented Target and Beam

B. Unoriented Target--Polarized Beam

C. Oriented Target--Unpolarized Beam

44

D. Oriented Target and Beam

V. APPENDIX

A. Complete Set of Matrices for any Spin-Rotation Group Representations

B. Complete Set of Matrices for any Spin-Lorentz Group Representations

C. Explicit "Boosting" of the Current-Spin One-half and Spin One

VI. REFERENCES

VII. ACKNOWLEDGMENTS 
Contributions of time-reversal noninvariant terms

in electron-nucleus elastic scattering

Loren Dell Krase

Under the supervision of R. H. Good

From the Department of Physics

lowa State University

The possibility of using elastic scattering of electrons from nuclei having spin one or greater to search for $T$ noninvariant effects in electromagnetic processes was investigated. In the one photon exchange approximation of the elastic scattering process, terms which are invariant under $P$ and PCT but noninvariant under $T$ may be explicitly included in the nuclear current for the interaction of these nuclei. The transformation properties of the various terms which may contribute to the current were developed and discussed within a manifestly covariant framework. The current was then specialized to a noncovariant form in the Breit (or brickwall) frame of the nucleus where the physical form factors of the nuclear current were easily identified. This noncovariant form was then used to determine tests which could be used to search for $T$ noninvariant effects.

Two restrictions were placed on the types of experimental tests which could be made: 1) Since the polarization tensors of large rank can be produced only with a large momentum transfer, or emission or absorption of radiation of large multipolarity, the polarization tensors were taken to be; at most, second rank; 2) to ensure that the results would be as statistically accurate as possible, the measurable quantities were taken to be the cruss sections and the elcctron beam polarizatinns: Within these restrictions, at least two types of tests are possible. If the nuclear target is completely described by a vector polarization only, with a component normal to the scattering plane, the cross section for the scattering of unpolarized electrons has an asymmetry if the electromagnetic interaction is noninvariant under $T$. If the nuclear target is completely described by a second rank tensor only, with components in the scattering plane, an initially unpolarized electron beam has a polarization after the

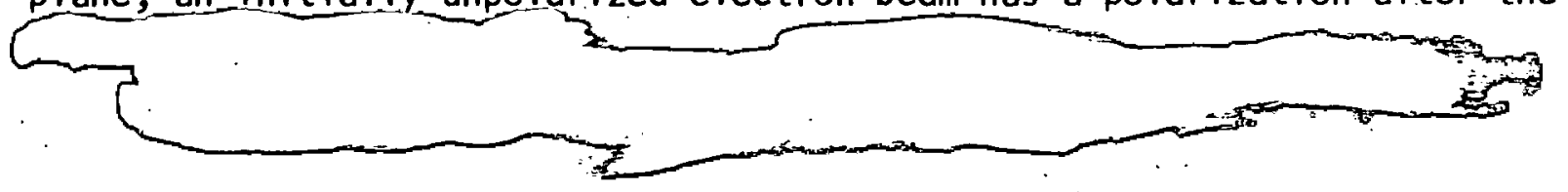


scattering if the electromagnetic interaction is noninvariant under $T$.

These tests, however, are not completely unambiguous. The polarization tensors of other rank may give a contribution which is not zero even when the electromagnetic interaction is invariant under $T$. In addition, the higher order processes may give a nonzero contribution even when the interaction is $T$ invariant, as is demonstrated for the test involving the asymmetry in the cross section. 


\section{INTRODUCTION}

The existence of $T$ noninvariant terms in the electromagnetic current of the hadrons was first proposed by Bernstein, Feinberg, and Lee ${ }^{l}$ to explain the observed violation of PC invariance in the decay $K_{L}^{0} \rightarrow \pi^{+} \pi^{-}$. After a review of the then existing data, they reached the conclusion that the invariance of the lepton electromagnetic current under $P, C$, and $T$, separately, was well established. However, the hadron current had been shown experimentally to be invariant only under PCT in general. Additionally, there seemed to be good evidence that the strong interactions conserved $P, C$, and $T$, separately, and that the electromagnetic interactions conserved $P$. Thus on the basis of the size of the PC violation parameter and the absence of experimental evidence to the contrary, they suggested that the source of the violation in the $K_{L}^{O}$ decay was a large violation of $C$ or $T$ in the electromagnetic interactions of the hadrons.

Since then, many ingenious experiments have been performed in an at tempt to detect such a violation of $C$ or $T$ invariance. Although the results have narrowed down the choice of the mechanism by which these effects may occur, they have all been consistent with no violation. In fact, the only unquestionable observation of PC noninvariance has been in the $K_{L}$ decay. The experimental tests may be divided into two categories; either a violation of $C$ or a violation of $T$ may be observed. The observation of $C$ violation must be confined to states of zero baryon number and have so far been indecisive. For baryons, direct tests can be made only of $T$ invariance. However, the antiunitarity of $T$ limits the observation of such effects to transition processes,

A good deal of effort has been expended in an attempt to observe an electric dipole moment of the neutron with results that are consistent with a zero moment to a high degree of accuracy. Although effects due to a nonzero electric dipole moment are forbidden by $T$, they are also forbidden by $P$. Hence this would seem to be more a verification of parity than a test of time-reversal.

Christ and Lee ${ }^{2}$ have indicated that a systematic test of $T$ invariance can be made by studying the inelastic scattering of charged leptons on nuclear targets. In particular, they have suggested observing the 
correlation function $\underset{\sim}{P}$ in $\left(\underset{\sim}{k} \times k^{\prime}\right)$ where $\underset{\sim}{P}$ in is the polarization vector of the initial nucleus and $k,{ }^{\prime}$ are, respectively, the initial and final momenta of the leptons. This function is odd under $T$ and therefore observance of a nonzero value would indicate a violation of $T$ invariance. The most recent data ${ }^{3}$, however, is consistent with no violation.

An alternative test of $T$ invariance, as was first noted for deuterium targets $^{4-8}$, can be provided by the observation of various correlation functions in the elastic scattering of charged leptons on nuclei with spin greater than one-half. For elastic scattering, phenomenological Hamiltonians which have $T$ noninvariant terms can be explicitly constructed. Thus specific statements can be made about how the $T$ noninvariant terms will affect the scattering. Parity, Hermiticity, and conservation of the nuclear current are sufficient to eliminate all terms which are noninvar$i$ ant under $T$ in the cases where the nuclear targets have spin zero or one-half. However, for spin greater than one-half these considerations are not as restrictive, and $i t$ becomes possible to observe $T$ noninvariant effects. The absence of $T$ noninvariant effects for the nucleons has been taken as a criticism of this approach; if the impulse approximation were exact, no violation effect would be observable. The impulse approximation, however, has been found to be limited to certain ranges of the scattering parameters and this criticism is apparently not completely valid. Results of experimental investigation of this test with a deuterium target ${ }^{9}$ have again been consistent with no violation.

From the foregoing, it appears that $T$ noninvariant effects, if they exist, are small. It would therefore seem appropriate to look for some experimental test which could determine the existence, or nonexistence, of these effects with a high degree of statistical confidence. Since it is possible to study the $T$ noninvariant terms in an explicit fashion for elastic scattering of electrons on nuclei, we will examine the possibility of using certain correlations arising in this scattering for such experimental tests. Because the scattering cross section and the electron polarization may presently be measured with a good deal of accuracy, we shall confine our considerations to correlations which are associated with these quantities. For the elastic scattering tests, however, difficulties 
arise due to the existence of "simulated" T noninvariant correlations 10 . These simulated effects arise from the interference of different order amplitudes. There is no reason not to expect this to occur for amplitudes of any order. And, al though the higher order amplitudes are depressed in accordance with their order, they may also be enhanced by resonance effects. Therefore, we also wish to compare the first order correlations with those that arise with the higher order processes.

In the first part of Chapter 11 , we construct the nuclear current, which is used to construct the phenomenological transition matrix, including the symmetry properties of the various terms, in a manner analogous to that used for the nucleon current; in the remainder we simplify the algebraic considerations and indicate the identification of the form factors with the static moments. Chapter 111 details the considerations needed to account for the correlations which may arise with the higher order processes. In Chapter iv, the various correlations are examined which are observable assuming invariance under $P$, Hermiticity, and current conservation in the one photon exchange approximation, and invariance under $P, T$, and PT for the higher order processes. 


\section{ONE PHOTON EXCHANGE APPROXIMATION OF ELASTIIC ELECTRON-NUCLEUS SCATTERING}

\section{A. Elements of Scattering Theory}

The approach to the scattering process which we wish to use here is in terms of the scattering matrix rather than through a development of Lagrangian field theory. Although the one-photon exchange approximation is most easily developed by way of covariant fields, the general higher order processes are not. We shall give a brief discussion of the parts of scattering theory which are relevant to both developments.

The scattering matrix has been extensively discussed in the literature, and we will not at tempt to extend this discussion. The scattering matrix $S$ is related to the transition matrix $\boldsymbol{J}$ by

$$
\langle f|s| i\rangle=\delta_{f i}-i(2 \pi)^{4} \delta^{4}\left(P_{f}-P_{i}\right) \eta\langle f|J| i\rangle,
$$

where $|i\rangle,|f\rangle$ are the initial and final states of the system, respectively (both as given here are in-states); $P_{i}$ and $P_{f}$ are, respectively, the total momenta of the initial and final states of the system; $\boldsymbol{N}$ is a normalization factor whose definition depends on the types of particles (boson or fermion) which are involved in the scattering but is unimportant for our purposes here. The difference between the treatment of the onephoton exchange and the higher order processes is in the development of $J$.

The correlations which-we wish to discuss are most easily developed in terms of density matrix ideas. We may define the density. matrix for the final state of the system in terms of the initial state density matrix by

$$
\sum \rho_{f}=\tau \rho_{i} \bar{\tau},
$$

where $\Sigma$ is a normalization factor which is included to make $\operatorname{Tr} \rho_{f}=$ $\operatorname{Tr} \rho_{i}=1$. The factors $T$ and $\bar{\tau}$ which are included on the right are matrices that are involved in the expansion of the transition matrix in terms of the in-fields and depend on the initial and final states of the system for the one-photon exchange approximation. For the higher order processes they may be defined directly in terms of the matrix elements of $\mathcal{J}$. 
B. Construction of the Current in the

$(0, s) \oplus(s, 0)$ Representation.

in the one photon exchange approximation of elastic electron-nucleus scattering the elements of the transition matrix are given by

$$
\langle f|\mathcal{J}| i\rangle=\mathrm{e}^{2} \mathrm{q}^{-2}\left\langle\mathrm{Q}^{\prime}, \mathrm{k}^{\prime}\left|g_{\mathrm{e} \mu}(0)\right| \mathrm{Q}, \mathrm{k}\right\rangle\left\langle\mathrm{P}^{\prime}, \mathrm{K}^{\prime}\left|g_{N \mu}(0)\right| \underset{\sim}{\mathrm{P}}, \mathrm{k}\right\rangle,
$$

where $f_{e}$ is the current operator of the electron and the electron states are denoted by $|p, k\rangle$ and $\left|p^{\prime}, k^{\prime}\right\rangle ; \delta_{N \mu}$ is the nuclear current operator and the nuclear states are denoted by $\left|\mathcal{P}^{\prime}, K^{\prime}\right\rangle$ and $\left|\mathcal{P}^{\prime}, K^{\prime}\right\rangle ; q^{2}$ is the square of the four-momentum transfer. We shall use units where $\hbar=c=1$. Latin indices range from one to three and Greek indices from one to four; $x_{4}=i$.

The expansion of the currents of the electron and nucleus in terms of in-fields may be made in a general fashion if we work with fields that are expanded in terms of $2(2 s+1)$ - component wave functions. The wave equations which these functions satisfy have been completely developed $11-13$. The quantization of each description can be carried out in the usual way with the introduction of creation/destruction operators for each of the allowed states 12,14 . The complete set of matrices which are needed in using this approach to the currents have been developed by Weinberg ${ }^{12}$, Williams ${ }^{15}$, Nelson and Good ${ }^{16}$, and in Appendix B. Electromagnetic form factors for arbitrary spin have previously been discussed by The is 17 using $(2 s+1)$ - component fields; however, these fields do not transform simply under $P$ or $C$. The present approach has the advantage that the fields transform simply under the discrete transformations. On the other hand, it also has the disadvantage that not all of the possible terms in the current are linearly independent. J. 0 . Eeg ${ }^{18}$ has also used the $2(2 s+1)$ component fields to discuss the electromagnetic interactions, but his discussion is not as extensive as the present one.

The spin s fields may easily be written in terms of the creation and destruction operators and the plane-wave eigenstates which we now define and develop. We differ with $\mathrm{Nelson}$ and $\mathrm{Good}^{14}$ only in the wave equation which the plane-waves are required to satisfy.

The plane-wave eigenstates $\psi_{p e k}$ involved in the expansion of the field can be written as 


$$
\Psi_{p \in k}=(2 \pi)^{-3 / 2} \mathrm{~m}^{\mathrm{s}} \mathrm{E}^{-\frac{1}{2}} v_{\in k}(\mathrm{p}) \exp [i(\underset{\sim}{\mathrm{x}}-\in E t)],
$$

where the physical momentum is $\underset{\sim}{p}$ and the energy is $E ; \epsilon$ is $+1(-1)$ for the particle (antiparticle). The functions $v_{e k}(\mathbb{Z})$, which we shall call'spinors' in analogy with the solutions of the Dirac equation, are related to rest frame spinors $v_{R \in k}$ by

$$
v_{\in k}(p)=\exp [s \in \underset{\sim}{\infty} \cdot(p / p) \operatorname{arctanh}(p / E)] v_{R \in k} .
$$

The exponential operator is the $(0, s) \oplus(s, 0)$ representation of a pure Lorentz transformation as given in Appendix B, Eq. (5.29) with

$$
\underset{\sim}{\tau}=-i \in(p / p) \operatorname{arctanh}(p / E) \text {, }
$$

and $\underset{\sim}{\alpha}$ as given in Eq. (5.30): The rest frame spinors are completely specified, except for an overall sign, by the following set of requirements:

$$
\begin{aligned}
& \beta v_{R \in k}=\epsilon v_{R \in k}, \\
& \left(C_{v_{R}, \epsilon, k}\right)^{*}=\epsilon^{2 s+1} v_{R,-\epsilon, k}, \\
& \gamma_{5} v_{R, \epsilon, k}=-(-1)^{s+\epsilon k} v_{R,-\epsilon,-k}, \\
& \underset{\sim}{\beta} v_{R \in k}^{e}=k v_{R \in k}, \\
& \beta s_{ \pm} v_{R, \epsilon, k}=[s(s+1)-k(k \pm \epsilon)]^{\frac{1}{2}} v_{R, \epsilon, k \pm \epsilon,},
\end{aligned}
$$

where the matrices $\beta, \mathcal{C}, \gamma_{5}$, and $s$ are matrices defined in Eq. $(5.30)$; $s_{ \pm}$is $\stackrel{s}{\sim} \underset{\sim}{f} \pm \mathbf{i} \cdot g$ where $\underset{\sim}{e}, \underset{\sim}{f}$, and $g$ form an orthogonal right-hand set of unit vectors. The normalization of these spinors is taken to be

$$
v_{R \in k}^{\dagger} v_{R \in \epsilon^{\prime} k^{\prime}}^{\dagger}=\delta_{\epsilon \in \epsilon^{\prime}} \delta_{k k^{\prime}} \text {. . }
$$

Making the identification of $\beta$ with $\gamma_{3}, 44 \ldots .4$ as in Eq. (5.34) and using

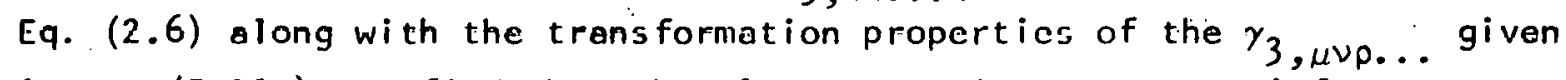
in Eq. $(5.31 \mathrm{c})$, we find that the plane-wave eigenstates satisfy

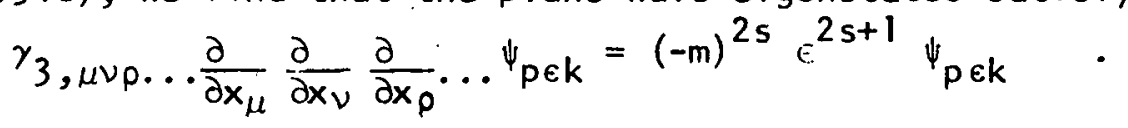

The plane-wave eigenstates also satisfy the Klein-Gordon equation as is apparent from Eq. (2.4). Any wave function packets which may be constructed from these plane-wave functions must also satisfy the Klein-Gordon equation. Therefore, the positive-definite Lorentz-invariant integral between two functions $\psi^{(\ell)}$ and $\psi^{(n)}$ may be defined by 


$$
\left(\psi^{(l)}, \psi^{(n)}\right)=-\frac{1}{2} i m^{-2 s} \int d x\left(\frac{\partial \bar{\psi}^{(l)}}{\partial t} \psi^{(n)}-\bar{\psi}^{(l)} \frac{\partial \psi^{(n)}}{\partial t}\right),
$$

where $\bar{\psi}$ is $\psi^{\dagger} \beta$. The normalization of the plane-wave eigenstates is such that

$$
\left(\psi_{p \in k}, \psi_{p^{\prime} \varepsilon^{\prime} k^{\prime}}\right)=\delta_{\varepsilon \epsilon^{\prime}} \delta_{k k^{\prime}} \delta\left(p-p^{\prime}\right) .
$$

The destruction and creation operators $a_{\epsilon k}(p)$ and $a_{\epsilon k}^{*}(p)$ (the asterisk denoting Hermitian conjugation in the Fock space) are postulated to satisfy the rules

$$
\begin{aligned}
& {\left[a_{\in k}\left(p_{1}\right), a_{\delta l}\left(p_{2}\right)\right]_{ \pm}=0} \\
& {\left[a_{\in k}\left(\sim_{\sim}\right), a_{\delta \ell}\left(\sim_{2}\right)\right]_{ \pm}=\delta_{k \ell} \in \delta\left(\sim_{\sim}-p_{2}\right),}
\end{aligned}
$$

where the upper (lower) sign is to be used for fermions (bosons). Also, it is postulated that any operator $a_{\epsilon k}(p)$ applied to the vacuum $|0\rangle$ gives zero and that all physical states are produced by applying the operators $a_{e k}^{*}(p)$ in any number to the vacuum.

The spin s field is taken to be

$$
\psi(x)=\int d p \sum_{k}\left[a_{1 k}(p) \psi_{p l k}+a_{-1 k}^{*}(p)\left\{-\gamma_{5}\right\}_{B} \psi_{p-l k}\right],
$$

where the notation $\left\{-\gamma_{5}\right\}_{B}$ indicates that the factor $-\gamma_{5}$, is to be included for bosons only, not for fermions. Using the plane-waves and the creation and destruction operators as defined above, it is possible to show that $\psi$ is causal. in the usual sense. From Eq. (2.11) we find that the fields satisfy the equation

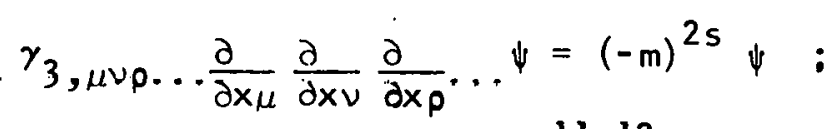

that is, the Joos-Weinberg equation ${ }^{11-12}$, which specializes to the Dirac equation for spin one-half.

The properties of the fields under space and time reflection and charge conjugation have been examined by Weinberg ${ }^{12}$ and $\mathrm{Nel}$ son and Good 14 and our definitions agree with theirs. Although we have defined the planewaves in a slightly different fashion than Nelson and Good, the difference is not sufficient to change the transformation properties of the planewaves. Therefore, we simply state their results for future reference. The parity operator is defined to be unitary and such that 


$$
\begin{aligned}
& P \psi(\underset{\sim}{x}, t) P^{-1}=\eta \beta \underset{\sim}{\psi(-\underset{\sim}{x}, t),} \\
& P|0\rangle=|0\rangle .
\end{aligned}
$$

The $a_{\varepsilon k}(Q)$ therefore transform as

$$
P a_{\epsilon k}(p) P^{-1}=\eta\{\epsilon\}_{F} a_{\epsilon k}(-p) \text {, }
$$

which follows from the property

$$
\beta \psi_{p \in k}(-\underset{\sim}{x}, t)=\epsilon \psi_{-p \in k}(\underline{x}, t) .
$$

The time-reversal operator is taken to be antilinear and antiunitary and such that

$$
T \psi(\underset{\sim}{x}, t) T^{-1}=n C \gamma_{5} \beta \psi(\underset{\sim}{x},-t),
$$

where $n$ is the parity signature. The plane-waves satisfy

$$
C \gamma_{5} \beta \psi_{p, \varepsilon, k} \underset{\sim}{(x,-t)}=(-1)^{s-k} \psi_{-p, \varepsilon,-k}^{*} \underset{\sim}{(x, t)} \text {, }
$$

which follows from Eqs. $(2.5),(2.6),(2.7)$ and $(2.8)$. Thus Eq. (2.22) gives

$$
T a_{\varepsilon, k}(p) T^{-1}=\eta(-1)^{s+k} a_{\varepsilon,-k}(-p) .
$$

The charge conjugation operator. is postulated to be unitary and to have the properties

$$
\begin{aligned}
& c \psi(x, t) c^{-1}=\left[C\left\{\gamma_{5}\right\}_{B} \psi(x, t)\right]^{*}, \\
& c|0\rangle=|0\rangle .
\end{aligned}
$$

The charge conjugation property of the plane-waves,

$$
\left[C_{\psi_{\varepsilon \in k}}\right]^{*}=\epsilon^{2 s+1} \psi_{-p,-\epsilon, k},
$$

follows from Eqs. (2.5) and (2.7) and the definition of $C$ as given in Appendix B. The transformation of the ${ }_{e k}(p)$ from Eq. $(2.25)$ is

$$
\text { c. } a_{e k}(p) c^{-1}=a_{-\epsilon k}(-p) \text {. }
$$

The transformation rule for the total reflection PCT, found by combining Eqs. $(2.18),(2.22)$, and $(2.25)$, is

$$
\operatorname{PCT} \psi(x, t) T^{-1} C^{-1} P^{-1}=\left[\left\{\gamma_{5}\right\}_{F} \psi(-x,-t)\right]^{*} \text {. }
$$


We are now in a position to give a general development of the current operators which appear in Eq. (2.3), including the transformation properties of the terms which may contribute. We shall restrict the terms that make up the current to be invariant under $P$ and $P C T$ and require that the current be conserved and be Hermitian. The first three requirements are well verified experimentally and are easily understood. The Hermiticity requirement is not as clear, but may be shown to be involved with the invariance of the scattering matrix under an antiunitary operation. Invariance under time-reversal, for example, requires that the transition matrix satisfy $T^{-1}=\sigma^{+}$. Invariance of the electromagnetic current requires that $T \delta_{\mu} T^{-1}=-\mathscr{\ell}_{\mu}$. Therefore, for the transition matrix as given in Eq. (2.3) we find $f_{e \mu} g_{N_{\mu}}=g_{e_{\mu}}^{+} f_{N_{\mu}}^{+}$. The current of the electron is well known from quantum electrodynamics to be invariant under $T$ and can be chosen to be Hermitian. Thus, the nuclear current is required to be Hermitian. On the other hand, if the nuclear current is Hermitian but not invariant under $T$, the transition matrix will not be invariant under $T$. Clearly only if the nuclear current is Hermitian will the current and the transition matrix simultaneously be either invariant or noninvariant under T. Similar considerations also hold for PCT.

The current operator may be written in terms of the fields as

$$
g_{\mu}(x)=: \bar{\psi}(x) J_{\mu} \psi(x): \text {, }
$$

where $\bar{\psi}=\psi^{\dagger} \beta$ and the dots indicate the normal product of the operators: Every term involving $a_{c k}^{*}\left(p_{1}\right)$ and $a_{b} l\left(p_{2}\right)$ is organized so that the $a^{*}$ occurs on the left of the $a$, with anticommutators for fermions and commutators for bosons being neglected in the process. $J_{\mu}$ is a matrix operator made up of the derivatives of the fields and the matrices defined in Appendix $B$, Eqs. (5.31). We shall use the derivatives in special combinations given by

$$
\begin{aligned}
& : \bar{\psi} Q_{\mu} M \psi:=i: \frac{\partial \psi}{\partial x_{\mu}} M \psi+\bar{\psi} M \frac{\partial \psi}{\partial x_{\mu}}:, \\
& : \bar{\psi} R_{\mu} M \psi:=i: \frac{\partial \bar{\psi}}{\partial x_{\mu}} M \psi-\bar{\psi} M \frac{\partial \psi}{\partial x_{\mu}}:,
\end{aligned}
$$

where $M$ is in general some matrix operator.

The transformation properties of the current may be found directly 
from Eq. (2.30) from the properties of the fields as given above. Alternatively, the transformation properties of the current may be found in terms of its matrix elements, using the transformation properties of the states which may be obtained directly from the properties of the creation operators. We shall use the latter approach since the matrix elements of the current will have central interest later.

The single particle or anti-particle state with quantum numbers $\underset{\sim}{\mathcal{P}} \in$, and $K$ is by definition

$$
|\underset{\sim}{P} \in K\rangle=a_{\in K}^{*} \underset{\sim}{*}(P)|0\rangle
$$

[We use upper case letters $(\underset{\sim}{P}, K)$ to agree with the definition of the nuclear states in Eq. (2.3).J The transformation of this state under space reflection is found from Eqs. (2.19) and (2.20) to be

$$
P|\underset{\sim}{P} C K\rangle=\eta\{C\}_{F}|-\underset{\sim}{P} \in K\rangle
$$

For time-reversal, there is a difference in thought on how the vacuum state transforms: It either is transformed to a conjugate space, in which case the fields are transformed to this space also, or is unchanged, in which case the fields remain in the original space. With either treatment, the relation between the matrix elements of the current is the same.

Therefore, using Eq. (2.24) we simply write

$$
T|\underset{\sim}{P} \in K\rangle=\eta(-1)^{S+K}|-\underset{\sim}{P}, \epsilon,-K\rangle
$$

The charge conjugate of this state is found easily from Eqs. (2.26) and $(2.28)$ to be

$$
6|\underset{\sim}{P} \in K\rangle=|-\underset{\sim}{P},-\epsilon, K\rangle \text {. }
$$

The effect of PCT on this state, found by combining Eqs. (2.34), (2.35), and $(2.36)$, is

$$
P C T .|P \in K\rangle=(-1)^{S+K}\{\epsilon\}_{F}|-P,-\epsilon,-K\rangle \text {. }
$$

The current given in Eq. (2.30) is said to be Hermitian if its matrix clements satisfy

$$
\langle a|\&| b\rangle=\langle b|\&| a\rangle^{*:},
$$

and

$$
\langle a|f \circ| b\rangle=\langle b|\delta \circ| a\rangle^{*} \text {, }
$$


where $|a\rangle$ and $|b\rangle$ are any two states and $g_{0}=-i 4_{4^{\circ}}$. If the current is invariant under space reflection then its matrix elements satisfy

$$
\left\langle a\left|g_{\mu}(\underset{\sim}{x}, t)\right| b\right\rangle=a_{\mu \nu}\left\langle P a\left|g_{\nu}(-\underset{\sim}{x}, t)\right| P b\right\rangle \text {, }
$$

where $a_{i j}=-n_{i j}, a_{i 4}=a_{4 i}=0$, and $a_{44}=1$. The states $|P a\rangle$ and $|P b\rangle$ are written for. $P|a\rangle$ and $P|b\rangle$, respectively. It should also be noted that if $\mathcal{\gamma}_{\mu}$ involves derivatives of the fields as appear in Eqs. (2.31) and (2.32), the differentials are also reflected. For example, if we

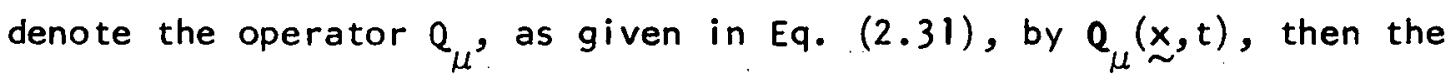
operator which appears on the right in Eq. (2.39) is $Q_{\mu}(-\underset{\sim}{-x}, t)$. Similar considerations hold for the other reflections also. The time-reversal invariance condition for the current is given by

$$
\left\langle a\left|g_{\mu}(\underset{\sim}{x}, t)\right| b\right\rangle=-\left\langle T a\left|g_{\mu}(\underset{\sim}{x},-t)\right| T b\right\rangle^{*},
$$

where the states $|T a\rangle$ and $|T b\rangle$ are written for $T|a\rangle$ and $T|b\rangle$, respectively. The same functional change of coordinates holds for the derivative operators as well as for the current. Charge conjugation invariance gives the relation

$$
\left.\left\langle a\left|g_{\mu}(\underset{\sim}{x}, t)\right| b\right\rangle=-\langle c a| g_{\mu} \underset{\sim}{x}, t\right)|c b\rangle,
$$

where $|C a\rangle$ and $|C b\rangle$ denote $C|a\rangle$ and $C|b\rangle$, respectively. The PCT relation follows from combining Eqs. (2.39), (2.40), and (2.41); and is given by

$$
\left\langle a\left|g_{\mu}(x, t)\right| b\right\rangle=a_{\mu \nu}\left\langle\text { PCT } a\left|g_{\nu}(-x ;-t)\right| P C T b\right\rangle^{*} \text {, }
$$

where $a_{i j}=-\delta_{i j}, a_{i 4}=a_{4 i}=0$, and $a_{44}=1$. The meaning of the states is clear from above.

If we take $|a\rangle$ and $|b\rangle$ to be the single particle states $\left.\left|p_{\sim}^{\prime}\right| K^{\prime}\right\rangle$ and $|P| K\rangle$, respectively, the matrix element of the current is

$$
\left\langle a\left|g_{\mu}\right| b\right\rangle=\Psi_{P} \mid k^{\prime},{ }_{\mu}^{j} \psi_{P I K},
$$

where the $\Psi_{P I K}$ are plane-wave eigenstates as given in Eq. (2.4). The matrix elements of the equations defining the derivative operators, (2.31) and $(2.32)$, are

$$
\left\langle a\left|: \bar{\psi} Q_{\mu} M \psi:\right| b\right\rangle=\left(P_{\mu}^{\prime}-P_{\mu}\right) \bar{\psi}_{P^{\prime} \mid K^{\prime}} M \psi_{P I K},
$$

and

$$
\left\langle a\left|: \bar{\psi} R_{\mu} M \psi:\right| b\right\rangle=\left(P_{\mu}^{\prime}+P_{\mu}\right) \bar{\psi}_{P^{\prime} I K^{\prime}} M_{P^{\prime K}} \cdot
$$


We define these combinations of the momenta as

$$
\begin{aligned}
& \mathrm{O}_{\mu}=\mathrm{P}_{\mu}^{\prime}-\mathrm{P}_{\mu}, \\
& \mathrm{R}_{\mu}=\mathrm{P}_{\mu}^{\prime}+\mathrm{P}_{\mu},
\end{aligned}
$$

and it will be these combinations which will be used in writing the matrix elements. Thus we write

$$
\left\langle a\left|g_{\mu}(x)\right| b\right\rangle=(2 \pi)^{-3} M^{2 s}\left(E^{\prime} E\right)^{-\frac{1}{2}} e^{-i Q \cdot x} u_{K}\left(P^{\prime}\right) J_{\mu}(Q, R) u_{K}(P),
$$

where we have used the definition of the plane-wave eigenstates and have replaced the particle spinors $v_{1 K}(P)$ by $u_{K}(P)$. What this is to be taken to mean is that the dependence of the current density operator on the combination of derivatives is to be replaced by the appropriate combinations of the momenta. As used here, $J_{\mu}$ is just a matrix built from $Q_{\mu}$ and $R_{\mu}$ and the matrices defined in Appendix B, Eqs. (5.31). The conditions on $\mathrm{J}_{\mu}$ which are imposed by Eqs. (2.38) through (2.42) are

$$
\begin{aligned}
& \bar{u}_{K^{\prime}}\left(P_{\sim}^{\prime}\right) \underset{\sim}{J}(Q, R) u_{K}(P)=\bar{u}_{K^{\prime}}\left(P_{\sim}^{\prime}\right)\left[\beta J_{\sim}^{\dagger}(-Q, R) \beta\right] u_{K}(P), \\
& \bar{u}_{K},\left(P^{\prime}\right) J_{O}(Q, R) u_{K}(P)=\bar{u}_{K^{\prime}}\left(P^{\prime}\right)\left[\beta J_{0}^{\dagger}(-Q, R) \beta\right] u_{K}(P), \\
& \left.\bar{u}_{K^{\prime}}\left(P_{\sim}^{\prime}\right) J_{\mu}(Q, R) u_{K}(P)=a_{\mu \nu} \bar{u}_{K^{\prime}}\left(P^{\prime}\right)\left[\beta J_{\nu}(a), a R\right) \beta\right] u_{K}(P),
\end{aligned}
$$

where $a_{i j}=-\delta_{i j}, a_{i 4}=a_{4 i}=0$, and $a_{44}=1$,

$$
\left.\bar{u}_{K}\left(P^{\prime}\right) J_{\mu}(Q, R) u_{K}(P)=-\bar{u}_{K^{\prime}}\left(P_{\sim}^{\prime}\right)\left[\beta \gamma_{5} C^{-1} J_{\mu}^{*}(-a Q,-a R) C \gamma_{5} \beta\right] u_{K} \stackrel{\sim}{\sim}\right),
$$

where $a_{i j}=\delta_{i j}, a_{i 4}=a_{4 i}=0$, and $a_{44}=-1$,

$$
\begin{aligned}
& \left.\bar{u}_{K^{\prime}}\left(P_{\sim}^{\prime}\right) J_{\mu}(Q, R) u_{K}(P)=-\bar{u}_{K^{\prime}}\left(P_{\sim}^{\prime}\right) \Gamma \beta\left\{\gamma_{5}\right\}_{B} C^{-1} J_{\mu}^{\dagger}(Q,-R) C\left\{\gamma_{5}\right\}_{B} \beta\right] u_{K}(P) \\
& \bar{u}_{K^{\prime}}\left(P_{\sim}^{\prime}\right) J_{\mu}(Q, R) u_{K}(P)=a_{\mu \nu} \bar{u}_{K^{\prime}}\left(P_{\sim}^{\prime}\right)\left[\left\{\gamma_{5}\right\}_{F} \beta J_{\nu}^{\dagger}(Q,-R) B\left\{\gamma_{5}\right\}_{F}\right] u_{K} \underset{\sim}{(P)}
\end{aligned}
$$


where $a_{i j}=-n_{i j}, a_{i 4}=a_{4 i}=0$, and $a_{44}=1$. The equations are numbered so as to agree with the Fock space conditions on the matrix elements. Eqs. (2.38') through (2.42') are found in a straightforward fashion from Eqs. (2.38) through (2.42) by using Eq. (2.43) and the properties of the spinors which follow from Eqs. (2.6) through (2.10). The matrix function on the left is to be expanded in terms of its arguments before the other operations are carried out. For a current that is Hermitian, Eqs. (2.40') and (2.42') may be written as

$$
\bar{u}_{K^{\prime}}\left(P^{\prime}\right) J_{\mu}(Q, R) u_{K}(P)=-a \nu_{\mu \nu} \bar{u}_{K^{\prime}}\left(\mathcal{L}^{\prime}\right)\left[\gamma_{5} \mathcal{C}^{-1} J_{\mu}^{\dagger}(a Q,-a R) C_{\gamma_{5}}\right] u_{K}(\mathcal{\sim}),
$$

where $a_{i j}=\delta_{i j}, a_{i 4}=a_{4 i}=0$, and $a_{44}=-1$, and

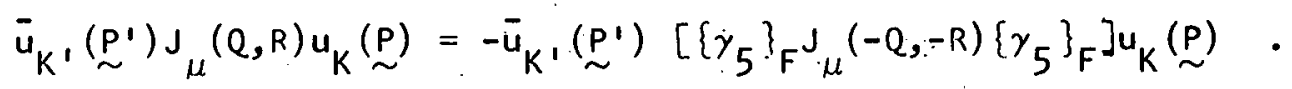

To explicitly construct $J_{\mu}$ and determine its transformation properties we first need to establish some properties of the matrices defined by Eqs. (5.31). We may consider the Hermiticity and space reflection invariance together if we write Eq.. $\left(2.38 b^{\prime}\right)$ in terms of $\mathrm{J}_{4^{\circ}}$. Thus since the matrices as defined are Hermitian, we need consider only $B \gamma_{A} \beta$. The properties of the matrices are given in Table 1.

For bosons, since the matrices are Hermitian, we need to consider only $B \gamma_{5} \mathrm{C}^{-1} \gamma_{A}^{\top} \mathrm{C}_{\gamma_{5}} \mathrm{~B}$ for both the time-reversal and charge conjugation invariance conditions. The properties of the matrices are given in Table 2. For fermions, however, we need to consider $\beta C^{-1} \gamma_{A}^{\top} C^{-1}{ }_{B}$ for the charge conjugation invariance condition. The matrices then have the properties given in Table 3 .

For the PCT invariance condition, we need to consider $\beta \gamma_{A}^{\beta}$ for bosons, which is identical with the Hermiticity and space reflection considerations. For fermions, we need to consider $\gamma_{5}{ }^{\beta \gamma}{ }_{A} \beta \gamma_{5}$, for which the matrices have the properties given in Table 4. If we use the extra condition that the current be Hermitian, we need to consider $\gamma_{5} \mathrm{C}^{-1} \gamma_{A}^{\top} \mathrm{C}_{5}$ for time-reversal, for which the matrices have the properties given in Table 5. For the 
Table 1. $\beta \gamma_{A}{ }^{\beta}$

$\beta \gamma_{1} \beta=\gamma_{1}$

$\beta \gamma_{2} \beta=-\gamma_{2}$

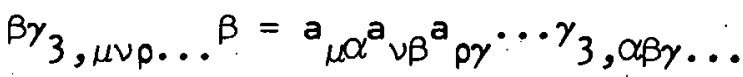

$\beta \gamma_{4, \mu \nu \rho . . .}{ }^{\beta}=-{ }_{\mu} \alpha^{a}{ }^{a} \beta^{a}{ }_{\rho \gamma} \cdots \gamma_{4, \alpha \beta \gamma} \ldots$

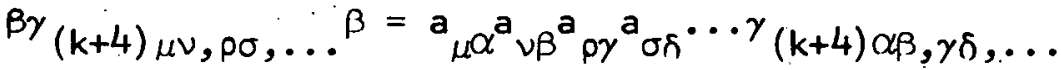

where: $\quad a_{i j}=-\delta_{i j}, a_{i 4}=a_{4 i}=0$, and $a_{44}=1$

Table 2. $B \gamma_{5} \mathrm{C}^{-1} \gamma_{\mathrm{A}}^{\top} \mathrm{C} \gamma_{5} \mathrm{\beta}$

$B \gamma_{5} C^{-1} \gamma_{1}^{\top} C \gamma_{5} \beta=\gamma_{1}$

$\mathrm{B \gamma}_{5} \mathrm{C}^{-1} \gamma_{2}^{\top} \mathrm{C} \gamma_{5} \beta=\gamma_{2}$

$B \gamma_{5} C^{-1} \gamma_{3, \mu \nu \rho . . . C \gamma_{5} \beta=\gamma_{3}, \mu \nu \rho . . .}$

$B \gamma_{5} C^{-1} \gamma_{4}^{\top}, \mu \nu \rho \ldots C \gamma_{5} \beta=-\gamma_{4}, \mu \nu \rho \ldots$

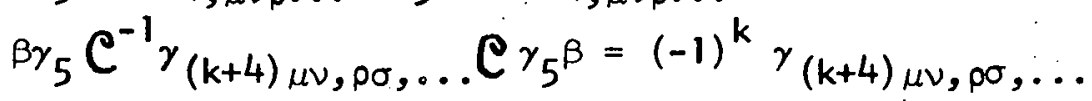

Table 3. $\beta C^{-1} \gamma_{A}^{\top} C_{B}$

$\beta C^{-1} \gamma_{1}^{\top} C_{\beta}=\gamma_{1}$

${ }_{\beta} \mathbb{C}^{-1} \gamma_{2}^{\top} C_{\beta}=\gamma_{2}$

$\beta C^{-1} \gamma_{3, \mu \nu \rho . . .} C^{\top}=-\gamma_{3}, \mu \nu \rho . .$.

$\beta C^{-1} \gamma_{4, \mu \nu \rho . . .}^{\top}{ }^{\top}=\gamma_{4, \mu \nu \rho . .}$

${ }_{\beta} C^{-1} \gamma_{(k+4) \mu \nu, \rho \sigma, \ldots}^{\top} C^{\beta}=(-1)^{k} \gamma_{(k+4) \mu \nu, \rho \sigma, \ldots}$ 
Table 4. $\quad \gamma_{5}{ }^{\beta \gamma}{ }_{A}{ }^{\beta \gamma} \gamma_{5}$

$$
\begin{aligned}
& \gamma_{5} \beta \gamma_{1} \beta \gamma_{5}=\gamma_{1} \\
& \gamma_{5}{ }^{\beta \gamma}{ }_{2} \beta \gamma_{5}=-\gamma_{2} \\
& \gamma_{5} \beta \gamma_{3, \mu \nu \rho \ldots}{ }^{\beta \gamma_{5}}=a_{\mu \sigma{ }^{a}{ }_{\nu \beta}{ }^{a} \rho \gamma} \cdots \gamma_{3, \alpha \beta \gamma} \ldots \\
& \gamma_{5} \beta \gamma_{4, \mu \nu \rho \ldots}{ }^{\beta \gamma_{5}}=a_{\mu \alpha} a_{\nu \beta}{ }^{a} \rho \gamma \cdots \gamma_{4, \alpha \beta \gamma} \ldots
\end{aligned}
$$

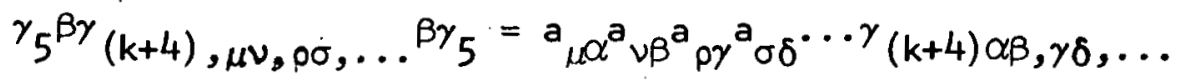

where $\quad a_{i j}=-\delta_{i j}, a_{i 4}=a_{4 i}=0$; and $a_{44}=1$

Table 5. $\gamma_{5} \mathrm{C}^{-1} \gamma_{A}^{\top} \mathrm{C}_{5}$

$$
\begin{aligned}
& \gamma_{5} C^{-1} \gamma_{1}^{\top} C \gamma_{5}=\gamma_{1} \\
& \gamma_{5} \mathrm{C}^{-1} \gamma_{2}^{\top} \mathrm{C}_{\gamma_{5}}=-\gamma_{2} \\
& \gamma_{5} C^{-1} \gamma_{3, \mu \nu \rho}^{\top} \ldots C^{\gamma_{5}}=(-1)^{2 s}{ }_{a}{ }_{\mu} \alpha^{a}{ }_{\nu \beta}{ }^{a}{ }_{\rho \gamma} \cdots \gamma_{3, \alpha \beta \gamma} \ldots
\end{aligned}
$$

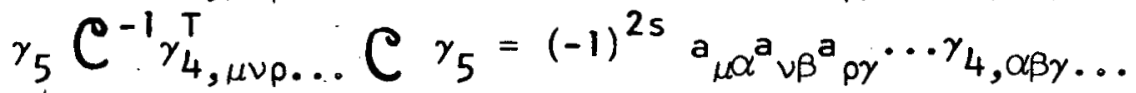

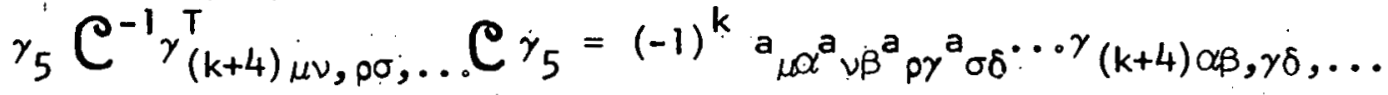

where

$$
a_{i j}=\delta_{i j}, a_{i 4}=a_{4 i}=0, a_{44}=-1
$$

Table 6. $\gamma_{5} \gamma_{A^{\prime}} \gamma_{5}$

$$
\begin{aligned}
& \gamma_{5} \gamma_{1} \gamma_{5}=\gamma_{1} \\
& \gamma_{5} \gamma_{2} \gamma_{5}=\gamma_{2} \\
& \gamma_{5} \gamma_{3}, \mu \nu \rho \ldots \gamma_{5}=-\gamma_{3}, \mu \nu \rho \ldots \\
& \gamma_{5} \gamma_{4}, \mu \nu \rho \ldots \gamma_{5}=-\gamma_{4}, \mu \nu \rho \ldots \\
& \gamma_{5}^{\gamma}(k+4) \mu \nu, \rho \sigma, \ldots \gamma_{5}=\gamma_{(k+4) \mu \nu, \rho \sigma, \ldots}
\end{aligned}
$$


PCT condition, the matrices are left unchanged for bosons; for fermions we need to consider $\gamma_{5} \gamma_{A} \gamma_{5}$ for which the matrices have the properties given in Table 6.

The various terms in the current which may be constructed for any spin, from the matrices and the momentum vectors $Q_{\mu}$ and $R_{\mu}$, are given in Table 7. The terms as given are Hermitian and all are invariant under PCT. Table 7 also gives their invariance properties under $P, C$, and $T$. If a term is invariant under a particular reflection, the appropriate condition holds as given by one of Eqs. (2.38') through (2.42') or (2'40'') or. $\left(2.42^{\prime \prime}\right)$, and we denote this by a "'+"; if it is noninvariant the appropriate condition with a minus sign multiplying the expression on the right in the equation holds, and we denote this by a "-".

The current is some linear combination of these terms multiplied by a real function (a form factor) of $Q^{2}$ only, since we are considering elastic processes. The matrix elements of these terms, however, are not all linearly independent. Since the matrix elements of the current are our ultimate concern, the linear independence of the matrix elements is of importance. The current we really wish to consider is a linear combination of terms whose matrix elements are linearly independent so that the form factors are non redundant. The wave equation requires that the spinors satisfy

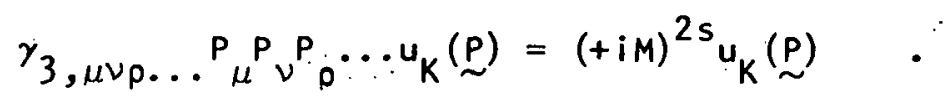

For low spin particles (essentially only spin one-half and spin one particles) this equation and the algebra of the matrices may be used to determine the linear relations between the various terms. For higher spins, however, the algebra becomes formidable and it seems expedient to search for other means of examining the linear independence of the terms.

Using Eq. (2.43), the conservation of the current can be shown to lead to the condition

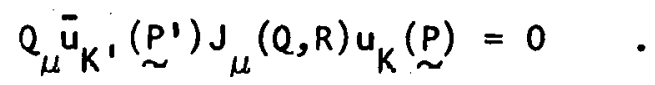

While some of the terms in the current obviously satisfy this condition, it is not at all clear how other terms do so. In general, this condition becomes involved with the linear independence of the terms. For example, 
Table 7. Possible current terms and their transformation properties

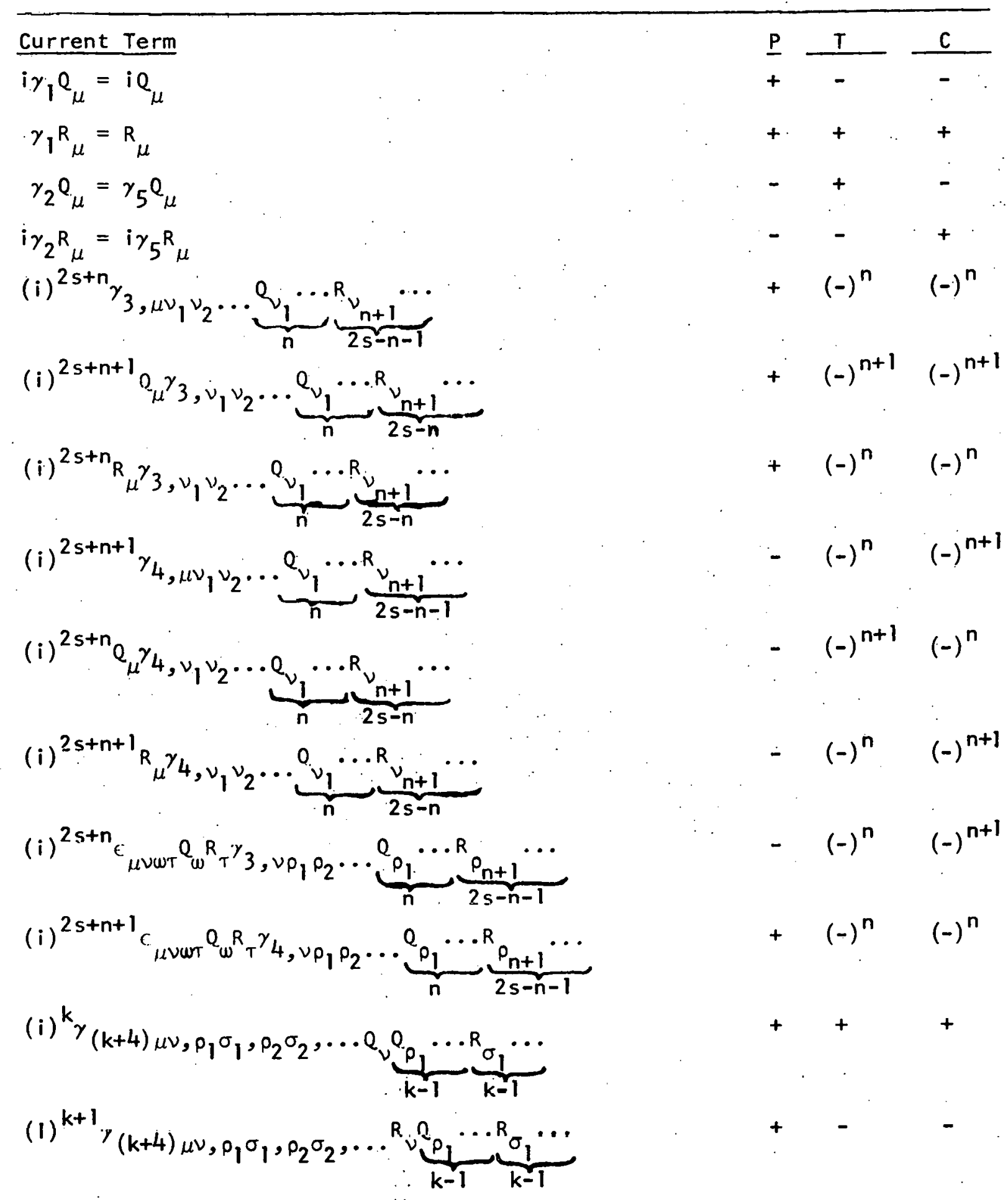


Table 7. (Continued)

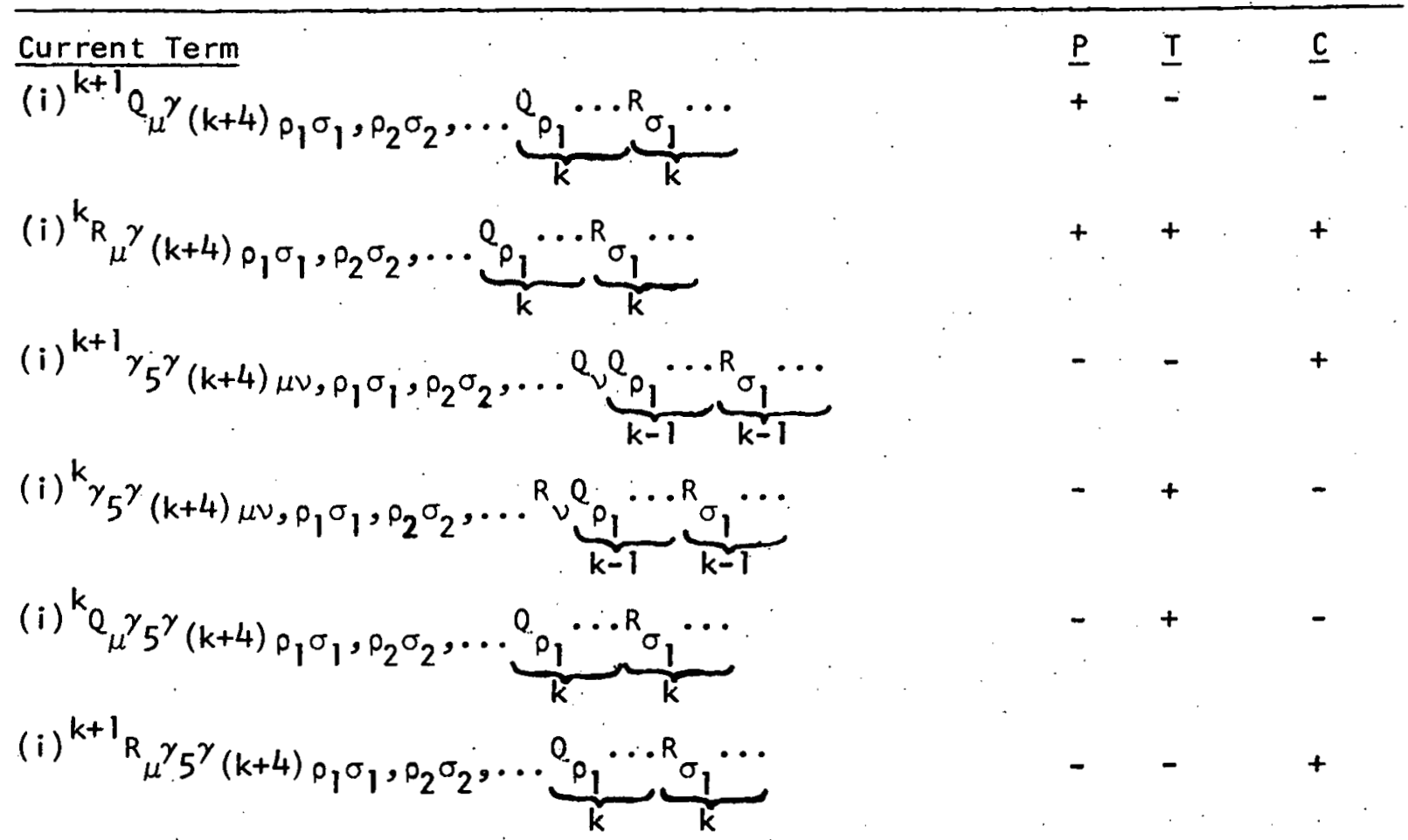

Only representative examples are given. The range on $n$ is such that the matrices are contracted with all possible combinations of $Q$ and $R ; k$ ranges from 1 to $2 \mathrm{~s}$. 
the 1 inear combination,

$$
\begin{aligned}
& \gamma_{3, \mu v_{1}} \ldots v_{2 s-1}\left(P^{\prime} v_{1} P^{\prime} \nu_{2} \ldots P^{\prime} v_{2 s-1}+P^{\prime} v_{1} P^{\prime} v_{2} \ldots P^{\prime} v_{2 s-2} P_{v_{2 s-1}}+\ldots\right. \\
& \left.\quad+P_{v_{1}} P_{v_{2}} \ldots P_{v_{2 s-1}}\right),
\end{aligned}
$$

may be shown to satisfy the conservation condition. But, except for spins one-half and one, this is a linear combination of some of the terms and the linear independence of the terms must be known before construction of the current is completed.

\section{c. The "Boosted" Current}

A general procedure exists which may be used in a simple fashion to examine the linear independence of the matrix elements of the current terms and the current conservation condition. The relation between the covariant spinors and the rest frame spinors, Eq. (2.5), is used to eliminate the momentum dependence from the spinors and to put this dependence into the current terms instead. This procedure, which we call "boosting" the current, was first suggested by Gourdin 19 as an especially simple means of relating covariant form factors to physical form factors for arbitrary spin. Gourdin and Micheli ${ }^{20}$ later used this procedure to discuss the covariant form factors in the Rarita-Schwinger formalism.

The boosting technique seems particularly convenient for discussing the current terms in the $(0, s) \oplus(s, 0)$ representation: The fields describe an object having unique spin and, as a consequence, the boosting is essentially an algebraic problem. The exponential involved in the definition of the covariant spinors may be written as a polynomial of degree $2 \mathrm{~s}$ in the components of $\alpha^{21}$. By using this polynomial form, the covariant matrices as defined in terms of the spin matrices, and the product rules of the spin matrices, we may determine the linear independence and the current conservation properties of the various terms in a straightforward fashion. This is carried out for spins one-half and one in Appendix $C$. We shall consider other more general properties of the boosted current here.

The matrix element on the right in Eq. (2.43) may be written in terms 
of the rest frame spinors as

$$
\bar{u}_{K^{\prime}}\left({\underset{\sim}{P}}^{\prime}\right) J_{\mu}(Q, R) u_{K} \underset{\sim}{(P)}=u_{R K}^{+}\left[B^{-1} \underset{\sim}{\left(P^{\prime}\right)} J_{\mu}(Q, R) B(\underset{\sim}{P})\right] u_{R K},
$$

where

$$
B(\underset{\sim}{)})=\exp [+s \alpha \cdot(P / P) \operatorname{arctanh}(P / E)]
$$

is the boost operator for particles. In deriving Eq. (2.46) from (2.43) we have used the property of the boosts, $B B_{\epsilon}^{\dagger} \beta=B_{\epsilon}^{-1}$, and $E q .(2.6)$. The matrix dependence of the quantity which occurs in the square brackets is a polynomial function of the matrices $\beta$ and $\gamma_{5}$ and the components $\underset{\sim}{\alpha}$ and $s$. But the matrices which give a nonzero contribution to the matrix element are those which commute with $\beta$. This follows easily from the properties of the rest frame spinors

$$
\frac{1}{2}(1+\beta) u_{R K}=u_{R K},
$$

so that the matrices which do not commute with $\beta$ are projected out. Thus we write

$$
u_{R K}^{+}\left[B^{-1}\left(\mathcal{\sim}^{\prime}\right) J_{\mu}(Q, R) B(\underset{\sim}{)}] u_{R K}=u_{R K}^{+} j_{\mu}(R, R, \underset{\sim}{s}) u_{R K},\right.
$$

since only $\beta$ and $\underset{\sim}{s}$ may give a nonzero contribution and $\beta$ may be el iminated using Eq. (2.6). We will refer to $j_{\mu}$ as the boosted current.

The reflection properties of the boosted current may be found from those of $\mathrm{J}_{\mu}$. The Hermiticity condition, Eqs. (2.38'), may first be written as

$$
\begin{aligned}
& u_{R K}^{\dagger}\left[B^{-1}\left(P^{\prime}\right) \underset{\sim}{J}(Q, R) B(\underset{\sim}{P})\right] u_{R K}=u_{R K}^{\dagger},\left[B ^ { - 1 } \left(\underset{\sim}{P} \underset{\sim}{J}(-Q, R) B\left(\underset{\sim}{\left(P^{\prime}\right)}\right]^{\dagger} u_{R K}\right.\right. \\
& u_{R K}^{\dagger}\left[B^{-1}\left(\mathcal{P}^{\prime}\right) J_{O}(Q, R) B(P)\right] u_{R K}=u_{R K}^{+}\left[B^{-1}(\mathcal{\sim}) J_{O}(-Q, R) B\left(\mathcal{P}^{\prime}\right)\right]^{\dagger} u_{R K},
\end{aligned}
$$

where we have used $B^{\dagger}(P)=B(P)$ in arriving at this form. The parity condition as follows from Ey. (2.39') is

$$
u_{R K}^{\dagger}\left[B^{-1}\left(P_{\sim}^{\prime}\right) J_{\mu}(Q, R) B(\underset{\sim}{P})\right] u_{R K}=a_{\mu \nu} u_{R K}^{+}\left[B^{-1}\left(-P_{\sim}^{\prime}\right) J_{\nu}(a Q, a R) B(-P)\right] u_{R K}
$$

where $a_{i j}=-\delta_{i j}, a_{i 4}=a_{4 i}=0$, and $a_{44}=1$. The time-reversal condition, 
Eq. $\left(2.40^{\prime}\right)$ becomes

$$
\begin{aligned}
& u_{R K^{\prime}}^{\dagger}\left[B^{-1}\left(P_{\sim}^{\prime}\right) J_{\mu}(Q, R) B(\underset{\sim}{P})\right] u_{R K} \\
& \quad=-u_{R K^{\prime} \gamma_{5}}^{\dagger} C^{-1}\left[B^{-1}\left(-P^{\prime}\right) J_{\mu}(-a Q,-a R) B(-P) J^{*} C \gamma_{5} u_{R K}\right.
\end{aligned}
$$

where $a_{i j}=\delta_{i j}, a_{i 4}=a_{4 i}=0$, and $a_{44}=-1$ and we have used $\mathcal{C} \underset{\sim}{\mathcal{Q}-1}=\alpha^{*}$ in moving the $B$ 's inside the square brackets. We may write the charge conjugation condition, Eq. $\left(2.41^{\prime}\right)$, as

$$
\begin{aligned}
u_{R K}^{+}\left[B^{-1}\left(P^{\prime}\right) J_{\mu}(Q, R) B(P)\right] u_{R K} \\
\left.\quad=-u_{R K}^{\dagger}\left\{\gamma_{5}\right\}_{B} C^{-1} f_{B}^{-1}(P) J_{\mu}(Q,-R) B\left(P^{\prime}\right)\right]^{\top} C_{\left\{\gamma_{5}\right\}_{B} u_{R K}} .
\end{aligned}
$$

The PCT condition, Eq. $\left(2.42^{\prime}\right)$, is then

$$
\begin{aligned}
& u_{R K}^{\dagger} B^{-1}\left(P^{\prime}\right) J_{\mu}(Q, R) u_{R K} \\
& \quad=a_{\mu \nu} u_{R K}^{\dagger}\left\{\gamma_{5}\right\}_{F}\left[B^{-1}(-P) J_{\nu}(Q,-R) B\left(-P^{\prime}\right)\right]^{\dagger}\left\{\gamma_{5}\right\}_{F} u_{R K}:
\end{aligned}
$$

If we take the current to be Hermitian, the time-reversal condition, Eq. $\left(2.40^{1.1}\right)$, may be written as

$$
\begin{aligned}
& u_{R K}^{+}, B^{-1}\left(P^{\prime}\right) J_{\mu}(Q, R) B(\underset{\sim}{P}) u_{R K} \\
& =-a_{\mu \nu} u_{R K}^{\dagger}{ }^{\prime} \gamma_{5} C^{-1}\left[B^{-1}(-\underset{\sim}{P}) J_{\mu L}(a Q,-a R) B\left(-P_{\sim}^{\prime}\right)\right]^{\top} C \gamma_{5} u_{R K},
\end{aligned}
$$

and the PCT condition, Eq. $\left(2.42^{\prime \prime}\right)$, is

$$
\begin{aligned}
& u_{R K}^{\dagger} B^{-1}\left(P^{\prime}\right) J_{\mu}(Q, R) B(P) u_{R K}
\end{aligned}
$$

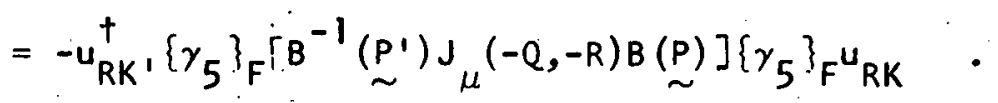

The reflection properties of the $j_{\mu}$ may be found from Eqs. (2.48) through (2.54): For the Hermiticity, parity, and time-reversal conditions, the arguments of the boost operators undergo the appropriate change so that the arguments of $j_{\mu}$ may be taken to agree with those of $J_{\mu}$. For example, for the Hermiticity condition, ${\underset{\sim}{\prime}}^{\prime}$ and $\underset{\sim}{P}$ are interchanged, which is in agreement with $Q_{\mu}$ being replaced by $-Q_{\mu}$ and $R_{\mu}$ by itself. Thus the Hermiticity condition may be written as 


$$
\begin{aligned}
& u_{R K \cdot j}^{\dagger}(Q, R, \underset{\sim}{s}) u_{R K}=u_{R K ' j^{j}}^{\dagger}(-Q, R, \underset{\sim}{s}) u_{R K}, \\
& u_{R K}^{\dagger} j_{O}(Q, R, s) u_{R K}=u_{R K}^{\dagger} j_{O}^{\dagger}(-Q, R, \underset{\sim}{s}) u_{R K},
\end{aligned}
$$

while the parity condition becomes.

$$
u_{R K}^{\dagger} j_{\mu}(Q, R, \underset{\sim}{s}) u_{R K}=a_{\mu \nu} u_{R K}^{\dagger} j_{\nu}(a Q, a R, \underset{\sim}{s}) u_{R K},
$$

where $a_{i j}=-\delta_{i j}, a_{i 4}=a_{4 i}=0$, and $a_{44}=1$. The time-reversal condition for a general current is then

$$
u_{R K}^{\dagger}, j,(Q, R, s) u_{R K}=-u_{R K}^{\dagger} \cdot j_{\mu}^{*}\left(-a Q,-a R,-s_{\sim}^{*}\right) u_{R K},
$$

where $a_{i j}=\delta_{i j}, a_{i 4}=a_{4 i}=0$, and $a_{44}=-1$. We have used $C^{-1} \mathcal{C}_{\sim}=-s_{\sim}^{*}$, the reality of $C$, and the commutation of $\gamma_{5}$ with $\underset{\sim}{s}$ to eliminate the $\gamma_{5}$ and $\mathcal{C}$ factors, For a Hermitian current, the time-reversal condition is

$$
u_{R K}^{\dagger}, j_{\mu}(Q, R, s) u_{R K}=a_{\mu \nu} u_{R K}^{\dagger}, j_{\nu}^{T}\left(a n,-a R,-s_{\sim}^{*}\right) u_{R K}
$$

with $a_{\mu \nu}$ as in Eq. (2.57).

The charge conjugation and PCT conditions on the boosted current are not as simple. The arguments of the boost operators, as they appear in Eqs. (2.51), (2.52), or (2.54), are not consistent with the reflection properties of $Q_{\mu}$ and $R_{\mu}$. Furthermore, because of the appearance of the $\gamma_{5}$ factor for either bosons or fermions, but not both, this difficulty cannot be removed unambiguously. Thus we must use the charge conjugation and PCT properties of the original terms in $J_{\mu}$ in order to know the properties of the corresponding boosted terms.

To discuss the physical form factors, we specialize to a definite reference frame. The appropriateness of the Breit (or brick-wall) frame of the nucleus for such a discussion has previously been pointed out ${ }^{22-24}$. The Breit frame is that frame for which $P_{\sim}^{\prime}=-P=\frac{1}{2} 2$, from which it follows for elastic scattering that $Z_{0}=0$ and $\underset{Z}{R}=\underset{\sim}{0}$. Thus the boosted current can only be a function of $\underset{\sim}{Q}, R_{0}=2 E$, where $E=\frac{1}{2}\left(Q^{2}+4 M^{2}\right)^{\frac{1}{2}}$, and of the spin matrices $\mathbf{s}$. Rather than at tempt to boost the covariant current termby-term for each spin, we use the rotational properties of the current, the functional dependence of the current in Breit frame, and the conserva- 
tion of the current to construct the boosted current in a general fashion. Al though the charge conjugation and PCT properties of the terms cannot be determined in this approach, we have shown the covariant terms to all be invariant under PCT. Thus it seems reasonable to assume all the terms in the boosted current are PCT invariant and therefore that the charge conjugation properties are equivalent to those of combined space-time reflection. In this respect, our approach is not too different than that commonly used in the helicity formalism and, in fact, it is not too surprising that our results are in agreement with those found for elastic scattering, using this formalism 25 .

If we impose the restrictions that the current be conserved and Hermitian and that it be invariant under space reflection, the most general form of the components of the current are

$$
\begin{aligned}
& j_{4}=i(4 \pi) \sum_{\text {even } L}(2 L+1)^{-\frac{1}{2}} \Gamma_{L}^{(c)}\left(Q^{2}\right) Y_{L M}^{*}(\hat{Q}) T_{L M}(s) ;: \\
& M
\end{aligned}
$$

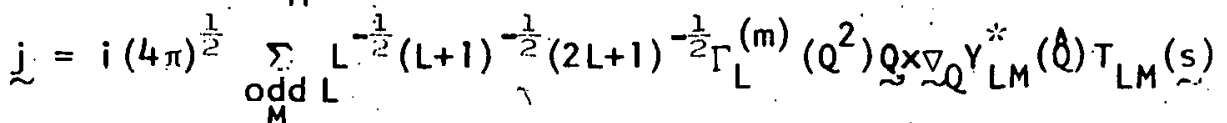

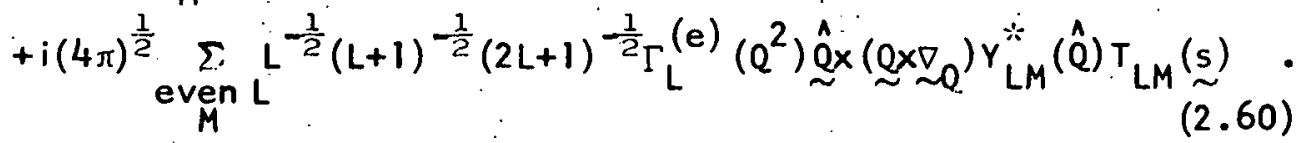

and

Here the $Y_{L M}$ are the usual spherical harmonics (we shall follow Rose's 26 definition) whose argument we have denoted by $\hat{Q}$ to emphasize that the angles are to be associated with the direction of $Q$. The $T_{L M}(s)$ are functionally the same as the spherical set of matrices discussed in Appendlx $A$; $L$ is limited in range to the maximum value of $2 \mathrm{~s}$ for which the $T_{L M}$ are nonzero. The normalization factors of $(4 \pi)^{\frac{1}{3}}$ and functions of $L$ are included for later convenience. No explicit dependence on $R_{0}$ has been given since it is clear from Eqs. (2.55) through (2.58), including the time-reversal invariance condition, that $R_{0}$ is left unchanged. Therefore we may think of the dependence on $R_{0}$, which may be taken to be a function of $Q^{2}$, as being included in $\Gamma_{L}$. The Hermiticity and space reflection properties may be found from Eqs. (2.55) and (2.56), respectively. The Hermiticity condition determines the reality of the various terms, and we have taken the 
$\Gamma_{L}$ to be real functions. Al though the most general form of the current components contains a sum on all values of $L$, space reflection invariance restricts the sum to only those values of $L$ as given.

The terms which are multiplied by the form factors $\Gamma_{L}^{(c)}$ and $\Gamma_{L}^{(m)}$ in Eqs. (2.59) and (2.60) are also invariant under time-reversal. The terms which are multiplied by the $\Gamma_{L}^{(e)}$, however, are noninvariant under timereversal and the $\Gamma_{L}(e)$ are restricted to be zero if the current is to be time-reversal invariant. To arrive at the time-reversal properties using either Eq. (2.57) or (2.58) we note that $\mathrm{T}_{L M}^{*} \underset{\sim}{*}(s)=(-1)^{M_{T}} \mathrm{~T}_{L,-M}\left(\mathrm{~s}_{\sim}^{*}\right)$ and that $T_{L M}(-s)=(-1)^{L} T_{L M}(s)$. With the additional restriction of time-reversal invariance, the boosted current as given here is the same as the current given by Scofield ${ }^{27}$ (except for an overall factor in each term which depends on $s$ only).

It should be noted that no time-reversal noninvariant terms occur for spin zero or one-half nuclei in Eqs. (2.59) and (2.60). Although this is a standard result which is easily proven covariantly, it is interesting to note that it also follows directly for the boosted current.

The three-vector part of the current as given by Eqs. (2.59) and (2.60) emphasizes the conservation condition. For many purposes, it is convenient to eliminate the derivatives and write the current in a form in which the multipolarity of the terms are more apparent. We do this by using the vector spherical harmonics which have been well discussed elsewhere 26,28 . We take the vector spherical harmonics to be defined by

$$
\underset{\sim}{Y} L M(\hat{\Omega})=\sum_{m} Y_{L, M-m}(\hat{\Omega}){\underset{\sim}{m}}_{m} C(L I J ; M-m, m),
$$

where $\quad \underset{\sim}{e} \pm 1=\mp \frac{1}{\sqrt{2}}(\underset{\sim}{e} \pm i \underset{\sim}{e})$,

$$
\underset{\sim 0}{e}=e_{z}
$$

and the C's are the Clebsch-Gordon coefficients as given by Rose ${ }^{26}$. It follows in a straightforward but lengthy fashion that

$$
\underset{\sim}{0 \times \nabla_{0}} Y_{L M}(\hat{Q})=i L^{\frac{1}{2}}(L+1)^{\frac{1}{2}}{\underset{\sim L L M}{L}}_{L}(\hat{Q}) \text {, }
$$


and

$$
\begin{aligned}
& \hat{Q} \times\left(\underset{\sim}{Q} \times \nabla_{Q}\right) Y_{L M}(\hat{Q})=-L^{\frac{1}{2}}(L+1)^{\frac{1}{2}}(2 L+1)^{\frac{1}{2}}\left[L^{\frac{1}{2}}{\underset{\sim}{\sim}, L+1, M}(\hat{Q})\right. \\
& \quad+(L+1)^{\frac{1}{2}} \underbrace{}_{L, L-1, M}(\hat{Q})] \quad \cdots
\end{aligned}
$$

It can also be shown from $Y_{L M}^{*}=(-1)^{M} Y_{L,-M}$ and Eq. (2.61) that

$$
\stackrel{\sim}{\sim} \underset{J L M}{*}=(-1)^{J+L+I-M} \stackrel{Y}{\sim} J, L,-M
$$

Therefore, Eq. (2.60) can be written as

$$
\begin{aligned}
& \dot{L}=(4 \pi)^{\frac{1}{2}} \sum_{\substack{\text { odd } L \\
\sum}}(2 L+1)^{-\frac{1}{2}} \Gamma_{L}^{(m)}\left(Q^{2}\right) \underset{\sim L L}{\gamma_{L}^{*}}(\hat{Q}) T_{L M}(s), \\
& -i(4 \pi)^{\frac{1}{2}} \sum_{\substack{\operatorname{even} L \\
\sum}}(2 L+1)^{-\frac{1}{2}} \Gamma_{L}^{(e)}\left(Q^{2}\right) \underset{\sim L M}{x}(\hat{Q}) T_{L M}^{*}(s),
\end{aligned}
$$

where we have introduced the special notation

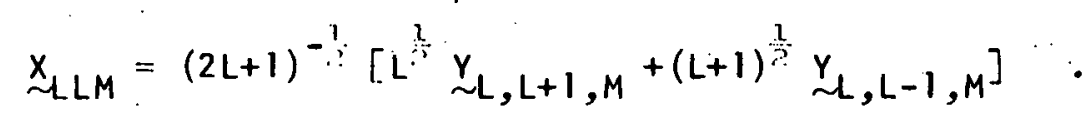

Thus, the factor of $(4 \pi)^{\frac{1}{2}}(2 L+1)^{-2} \Gamma_{L}\left(Q^{2}\right) T_{L M}(s)$ appears as a multipolar "component" of the current. This may also be shown by actually projecting out this factor. The vector spherical harmonics are orthonormal in the sense that

$$
\int \check{\sim}_{J L M}^{*} \underline{\sim}_{J} L_{L}{ }^{\prime} M^{\prime} d \hat{\Omega}=\delta_{J J^{\prime}} \delta_{L L^{\prime}} \delta_{M M^{\prime}}
$$

By using this orthonormality property and that of the $Y_{L M}$ we find

$$
\begin{aligned}
& (4 \pi)^{\frac{1}{2}}(2 L+1)^{-\frac{1}{2}} \Gamma_{L}^{(c)}\left(Q^{2}\right) T_{L M}(s)=\int-i j_{L} Y_{L M}(\hat{0}) d \hat{O} \cdots, \\
& (4 \pi)^{\frac{1}{2}}(2 L+1)^{-\frac{1}{2}} \Gamma_{L}^{(m)}\left(0^{2}\right) T_{L M}(s)=\int \dot{L} \cdot{\underset{\sim}{L L M}}_{L M}(\hat{Q}) d \hat{n},
\end{aligned}
$$

and

$$
(4 \pi)^{\frac{1}{3}}(2 L+1)^{-\frac{1}{?}} \Gamma_{L}^{(e)}\left(Q^{2}\right) T_{L M}(s)=i \cdot \int \dot{L} \cdot \underset{\sim}{x} L L M(\hat{Q}) \mathrm{d} \hat{Q} \text {. }
$$

The boosted current takes an especially simple form if we take. $P$ to define the z-axis. In that case, since $P+-\frac{1}{2}$,

and

$$
Y_{L M}(\hat{Q})=(4 \pi)^{-\frac{1}{2}}(21 .+1)^{\frac{1}{2}}(-1)^{L} \delta_{M 0}
$$

$$
\underset{\sim}{Y} L L M(\hat{Q})=(4 \pi)^{-\frac{1}{2}}(2 L+1)^{\frac{1}{2}}(-1)^{L} \sum_{m} \underset{\sim m}{e} C(L 1 J ; 0, m) \delta_{M-m, 0}
$$


An evaluation of the sum in the $\mathcal{X}_{J L M}$ using the explicit values of the C's leads to

$$
\underset{\sim}{\mathcal{Y} L M}(\hat{Q})=(4 \pi)^{-\frac{1}{2}}\left(L+\frac{1}{2}\right)^{\frac{1}{2}}(-1)^{L+1}\left(\delta_{M,+1} \stackrel{\sim}{\sim}+1^{-\delta_{M},-1} \underset{\sim-1}{e}\right),
$$

and

$$
{\underset{\sim}{L L M}}_{(\hat{Q})}(\hat{a})(4 \pi)^{-\frac{1}{2}}\left(L+\frac{1}{2}\right)^{\frac{1}{2}}(-1)^{L+1}\left(\delta_{M,+1} e_{+1}+\delta_{M,-1} e_{-1}\right)
$$

The components of the boosted current are

$$
j_{4}=i \underset{\text { even } L}{\Sigma} \Gamma_{L}^{(c)}\left(Q^{2}\right) T_{L O} \stackrel{(s)}{\sim},
$$

and

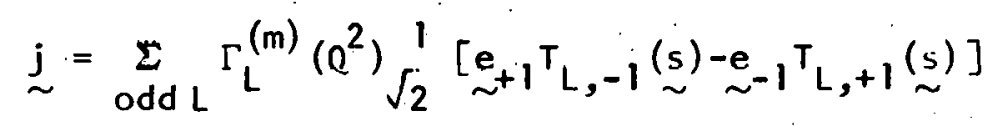

$$
\begin{aligned}
& -i \underset{\text { even } L}{\Sigma} \Gamma_{L}^{(e)}\left(Q^{2}\right) \underset{\sqrt{2}}{1}\left[\sim_{\sim+1}^{e} T_{L,-1} \stackrel{(s)}{\sim} \underset{\sim}{e} 1^{T} L,+1 \underset{\sim}{(s)}\right]
\end{aligned}
$$

The $x$ - and $y$-axes may be chosen from other kinematical considerations. We are still free to make this choice since the appearance of the unit vectors in Eq. (2.67) is a consequence only of the way we have defined the vector spherical harmonics.

An alternative approach to an understanding of the form factors, which is often used instead of starting with the current defined in momentum space, is to start with the current operator in coordinate space and to make a multipole expansion of the charge and current distributions. This approach may be well understood in the context of classical electrodynamics ${ }^{29}$, but it has also been used by Blatt and Weisskopf ${ }^{30}$ to define the static moments quantum mechanically. The multipole expansions of the charge and current operators are lusllally written as

$$
\begin{aligned}
& M_{L M}(Q)=i^{L} \int j_{L}(O r) Y_{L M}(\hat{r}) p(r) d^{3} r, \\
& T_{L M}^{(m)}(Q)=i^{L} Q^{-1} \int \underset{\sim}{J}(r) \cdot \nabla x j_{L}(Q r) \underset{\sim L L M}{Y}(\hat{r}) d^{3} r,
\end{aligned}
$$

and

$$
T_{L M}^{(e)}(Q)=i^{L} \int \underset{\sim}{J}(r) \cdot j_{L}(Q r) \underset{\sim L L M}{V}(\hat{r}) d^{3} r
$$

where $Q=|Q|$ and the $j_{L}(Q r)$ are the spherical Bassel functions which remain well defined at the origin. These multipole operators have been used to discuss both elastic scattering 31,32 and inelastic scattering 32,33 
of electrons on nuclei. The connection between these operators and the static moments for elastic scattering has also been made and are well discussed by überall and luginzius ${ }^{32}$, and Überal1 ${ }^{34}$. The functions

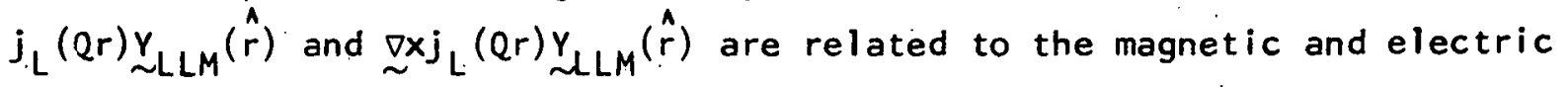
multipole fields in the solenoid guage by

and $\quad \underset{\sim}{\nabla} j_{L}(Q r) \underset{\sim L L M}{Y}(\hat{r})=i L^{\frac{1}{2}}(L+1)^{\frac{1}{2}} \underset{\sim}{Q A M}(e)$

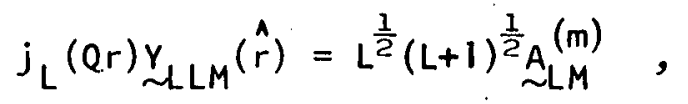

where

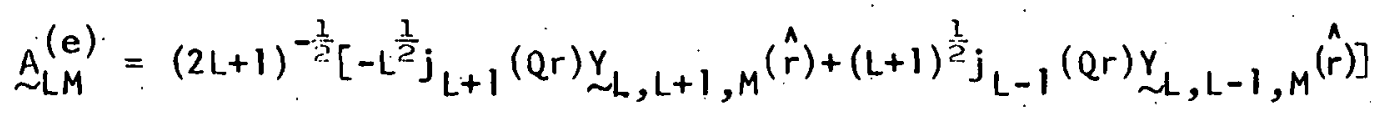

we may take

and

$$
\begin{aligned}
& (4 \pi)^{\frac{1}{2}} \Gamma_{L}^{(c)}=\left(s\left\|M_{L}\right\| s\right), \\
& (4 \pi)^{\frac{1}{2}} \Gamma_{L}^{(m)}=\left(s\left\|T_{L}^{(m)}\right\| s\right),
\end{aligned}
$$

where the quantities on the right are the reduced matrix elements of the multipole operators. This relation follows in a straightforward fashion after setting the multipole operators defined in Eqs. (2.65a) through (2.65c) equal to those defined in Eqs. (2.68a) through (2.68c), respectively. We will refer to the $\Gamma_{L}^{(c)}$ as the charge form factors, the $\Gamma_{L}^{(m)}$ as the magnetic form factors, and the $\Gamma_{L}(e)$ as the electric form factors.

In the following we shall assume that the current as given by Eqs. (2.66) and (2.67) may be found directly from the covariant current which is constructed from terms which are linearly independent and satisfy all the conditions. which were imposed on the boosted current; that is, current conservation, Hermiticity, and space reflection invariance. From this viewpoint, the covariant form factors are secondary; we identify the appropriate linear combination of them with the physical form factors by means of the boosting technique, but otherwise we simply require that they be covariant scalers. We will use the boosted current given by Eqs. (2.66) and (2.67) and we will take the $\Gamma_{L}$ to be physical form factors in our discussion of the correlation functions in Chapter IV. 


\section{HIGHER ORDER APPROXIMATIONS OF THE ELASTIC SCATTERING PROCESS}

As was mentioned in the introduction, the higher order processes must be taken into account for the elastic processes, since they have been shown for spin one-half nuclei to give rise to correlations which "simulate" first order time-reversal noninvariant correlations ${ }^{10}$. These correlations were found to be due to the interference of the first and second order terms and occur when the nucleon is polarized initially. Presumably, the same effects may occur for nuclei having any nonzero spin and may arise with amplitudes of any order. The higher order correlations should be calculable in principle if the exact nucleusphoton vertex were described by the phenomenological current as constructed in Chapter 11. However, at least at present, a quantum electrodynamics exists only for spin zero, one-half, and one nuclei in the formalism we are using. In addition, difficulties arise because of the other interactions of the nucleus so that it does not seem likely that each vertex in a many photon process is correctly described by the onephoton vertex. Therefore, we turn our attention to other means of discussing the higher order processes.

The Jacob and Wick helicity formalism 35 provides a very suitable alternative for discussing the types of correlations which may arise for a general order process. The description of the interaction in terms of electron-photon and nucleus-photon vertices is replaced by a general kinematical description which is taken to depend on the quantum numbers of the initial and final states nf the electron and nucleus only. Instead of attempting to isolate the parts of the interaction associated with each particle in the initial and final states, the matrix elements of the transition matrix are used directly. The quantities of interest are the amplitudes for the reaction $a+b \rightarrow c+d$, given by

$$
f\left(\lambda_{c} \lambda_{d} ; \lambda_{a} \lambda_{b}\right)=(2 p)^{-1} \sum_{j}(2 J+1)\left\langle\lambda_{c} \lambda_{d}\left|\mathcal{J}^{J}\right| \lambda_{a} \lambda_{b}\right\rangle{ }^{j}{ }_{\lambda \mu}^{j}(\theta),
$$

in the c.m. frame; $\lambda_{i}$ is the helicity of the $i$ th particle. Here $p$ is the size of the relative momentum, $8 J^{J}$ is the $j$ th partial wave contribution 
to the transition matrix, $\theta$ is the c.m. scattering angle, and $\lambda=\lambda_{a}-\lambda_{b}$, $\mu=\lambda_{c}-\lambda_{d}$. In arriving at Eq. (3.1), we have taken the direction of the nucleus' initial momentum to define the $z$-axis and the scattering plane to be the $x y-p l a n e$. The derivation of this expression is given by Jacob and Wick.

The amplitudes as given by Eq. (3.1) are all linearly independent, but space reflection and time-reversal invariance are known to lead to linear relations between the amplitudes. Since this places important restrictions on the correlations which we wish to consider, we will repeat the linear relations between the amplitudes here. The derivation of these relations is given by Jacob and Wick. Parity conservation leads to the relation

$$
\left\langle-\lambda_{c},-\lambda_{d}\left|J^{J}\right|-\lambda_{a},-\lambda_{b}\right\rangle=\left\langle\lambda_{c} \lambda_{d}\left|J^{J}\right| \lambda_{a} \lambda_{b}\right\rangle
$$

since we are considering an elastic process. Thus Eq. (3.1) becomes

$$
f\left(\lambda_{c} \lambda_{d} ; \lambda_{a} \lambda_{b}\right)=(2 p)^{-1} \sum_{J}(2 J+1)\left\langle-\lambda_{c},-\lambda_{d}\left|J^{\top}\right|-\lambda_{a},-\lambda_{b}\right\rangle d_{\lambda \mu}^{J}(\theta)
$$

The d's can be shown ${ }^{26}$ to have the symmetry properties

$$
d_{\lambda \mu}^{J}(\theta)=d_{-\mu,-\lambda}^{J}(\theta)=(-1)^{\lambda-\mu} d_{\mu \lambda}^{J}(\theta),
$$

which leads to the relation

$$
f\left(\lambda_{c} \lambda_{d} ; \lambda_{a} \lambda_{b}\right)=(-1)^{\lambda-\mu} f\left(-\lambda_{c},-\lambda_{d} ;-\lambda_{a},-\lambda_{b}\right)
$$

where $\lambda=\lambda_{\mathrm{a}}-\lambda_{\mathrm{b}}$ and $\mu=\lambda_{c}-\lambda_{\mathrm{d}}$. Time-reversal invariance leads to the relation

$$
\left\langle\lambda_{a} \lambda_{b}\left|\mathcal{J}^{J}\right| \lambda_{c} \lambda_{d}\right\rangle=\left\langle\lambda_{c} \lambda_{d}\left|\mathcal{J}^{J}\right| \lambda_{a} \lambda_{b}\right\rangle
$$

so that Eq. (3.1) becomes

$$
f\left(\lambda_{c} \lambda_{d} ; \lambda_{a} \lambda_{b}\right)=(2 p)^{-1} \sum_{J}(2 J+1)\left\langle\lambda_{a} \lambda_{b}\left|J^{J}\right| \lambda_{c} \lambda_{d}\right\rangle d_{\lambda \mu}^{J}(\theta)
$$

With the use of the symmetry relations given in Eq. (3.3), this leads to the relation

$$
f\left(\lambda_{c} \lambda_{d} ; \lambda_{a} \lambda_{b}\right)=(-1)^{\lambda-\mu} f\left(\lambda_{a} \lambda_{b} ; \lambda_{c} \lambda_{d}\right),
$$


where $\lambda$ and $\mu$ are the same as in Eq. (3.4). Our expressions for the 1 inear relations between the $f^{\prime} s$, Eqs. (3.4) and (3.6), differ with those given by Jacob and Wick, since we have chosen the scattering plane as the $x z-p l a n e$. Space reflection and time-reversal invariance combined leads to the linear relation

$$
f\left(\lambda_{c} \lambda_{d} ; \lambda_{a} \lambda_{b}\right)=f\left(-\lambda_{a},-\lambda_{b} ;-\lambda_{c},-\lambda_{d}\right),
$$

since $(\lambda-\mu)$ must be an integer for elastic scattering.

The amplitudes given in Eq. (3.1) may be used directly to calculate the sort of correlation functions we are interested in. The relation between the initial and final density matrices of the system is

$$
\Sigma \rho_{f}^{(0)}=f \rho_{i}^{(0)} f^{+} \text {, }
$$

which may be taken to be a boosted form of Eq. (2.2). The f's are matrices which are defined by

$$
u_{R \lambda_{c}}^{+} u_{R \lambda_{d}}^{+} f u_{R \lambda_{a}} u_{R \lambda_{b}}=f\left(\lambda_{c} \lambda_{d} ; \lambda_{a} \lambda_{b}\right) \text {, }
$$

where the rest frame spinors have been chosen to be helicity eigenstates. The quantity on the right is to be identified with the f's defined by Eq. (3.1): The density matrices of the system, which appear in both Eq. (2.2) and (3.8), may be taken to be a direct product of the density matrices for the electron and nucleus beams if the initial and final systems are assumed to be noninteracting. The density matrices which appear in Eq. (2.2) are covariant; that is, the density matrix which describes a beam of particles is to be expanded in terms of the covariant matrices for particles of that spin and covariant polarization tensors as has been carried out; for example, for spin three-halves 36 . The density matrices which appear in Eq. (3.8) are rest frame quantities; that is, they may be expanded in terms of the $T_{L M}(s)$ as developed in Appendix $A$ and rest frame polarization tensors which we take to be the statistical tensors as introduced by Fano 37 and further discussed by Biedenharn 38 . The connection between the covariant density matrix and the rest frame density matrix may be established by writing both in terms of the appropriate spinors: The covariant 
density matrix may be written as

$$
\rho=\sum_{K} c_{K} u_{K}(\stackrel{P}{\sim}) \bar{u}_{K}(P),
$$

where the $c_{K}$ are invariant weighting factors. In terms of the boost operators and the rest frame spinors this may be written as

$$
\rho=B(\underset{\sim}{P})\left[\sum_{K} c_{K} u_{R K} u_{R K}^{\dagger}\right] B^{-1}(P)
$$

The rest frame density matrix may be written as

$$
\rho^{(0)}=\sum_{K} c_{K} U_{R K} U_{R K}^{\dagger},
$$

so that $\rho=B(\underset{\sim}{P}) \rho^{(0)} B^{-1} \underset{\sim}{(P)}$.

(The expansion of the density matrix defined by Eq. (3.11) in terms of the statistical tensors involves the $2(2 s+1)$ - square spin matrices as defined in Eq. (5.30); however, they occur in the same functional form as the $T_{L M}$ discussed in Appendix $A$. This simply requires that a different normalization of the density matrix be used.)

To establish the connection between Eq. (3.8) and (2.2), we use Eq. (3.12) to boost the initial and final density matrices. To carry this out explicitly for the scattering process we are considering, we write

$$
T=\tau^{\top} e_{N},
$$

where $\tau_{e}$ and $\tau_{N}$ are parts which connect the initial and final states of the electron and nucleus, respectively.

The expansion of the covariant density matrix as given in Eq. $(3,10)$ suggests that we take

$$
\bar{T}=\left(\beta \tau^{+} e^{\beta}\right)\left(\beta T_{N}^{\dagger} \beta\right),
$$

where the daggers denote the matrix Hermitian conjugate and the factors of $\beta$ are the matrices $\beta$ of appropriate dimensionality.

We may argue the appropriateness of this factorization with the following considerations: The restriction we place on $T$ is that 


$$
\bar{u}_{\lambda{ }^{\prime}} \stackrel{\left(P^{\prime}\right) \bar{u}_{\lambda^{\prime}}\left(P_{N}^{\prime}\right) \tau u}{\sim} \lambda_{N} \stackrel{(P)}{\sim} u_{\lambda_{e}} \stackrel{(P)}{\sim}=f\left(\lambda^{\prime} e^{\lambda^{\prime}}{ }_{N} ; \lambda_{e} \lambda_{N}\right),
$$

in agreement with Eq. (3.9). We have used the helicity quantum numbers as spin projection values to label the covariant spinors, and the spinors are to be evaluated in the c.m. frame in agreement with the definition of the f's. Therefore, we may write

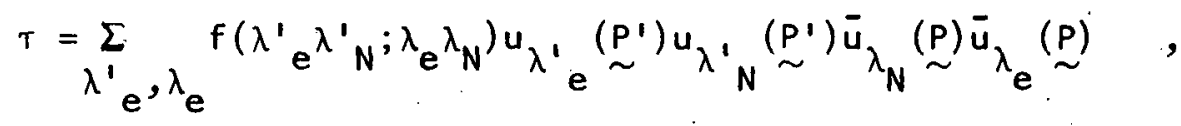

$$
\begin{aligned}
& \lambda^{\prime}{ }_{N}, \lambda_{N}
\end{aligned}
$$

and Eq. (3.14) follows from the orthonormality of the spinors. Thus, each term in the sum in Eq. (3.15) factorizes as indicated in Eq. (3.13a).

With the use of this factorization, Eq. (2.2) may therefore be written as

$$
\sum \rho_{f_{e}} \otimes \rho_{f_{N}}=\left(\tau_{e} \rho_{i} e^{\bar{\tau}} e\right) \otimes\left(\tau_{N} \rho_{i}{ }_{N} \bar{T}_{N}\right)
$$

Use of Eq. (3.6) results in

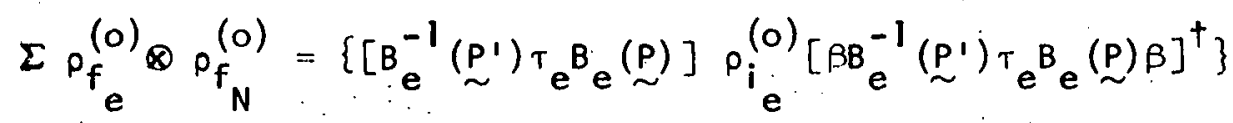

$$
\begin{aligned}
& \otimes\left\{\left[B_{N}^{-1}\left(P^{\prime}\right) \tau_{N} B_{N} \stackrel{(P)}{\sim}\right] \rho_{i_{N}}^{(0)}\left[\beta B_{N}^{-1}\left(P^{\prime}\right) \tau_{N} B_{N} \stackrel{(P) B}{\sim}\right]^{\dagger}\right\},
\end{aligned}
$$

where we have moved the final state boost operators to the right-hand side of Eq. (3.17) and used the properties $B^{\dagger}=B$ and $\beta B^{\dagger} \beta=B^{-1}$. Since we are using the density matrices for particle states only, the density matrices satisfy

$$
\frac{1}{2}(1+\beta) \rho^{(0)} \frac{1}{2}(1+\beta)=\rho^{(0)},
$$

as is clear from the expanded form given in Eq. (3.11). Therefore we write Eq. $(3.17)$ as

$$
\Sigma \rho_{f}^{(0)} \otimes \rho_{f_{N}}^{(0)}=\left[f_{e} \rho_{i}^{(0)} f_{e}^{\dagger}\right] \otimes\left[f_{N} \rho_{i}^{(0)} f_{N}^{\dagger}\right],
$$

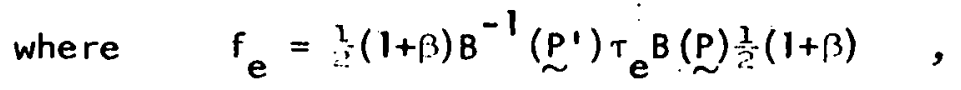

and a similar equation holds between $f_{N}$ and $T_{N}$. (The factors of $1+\beta$ ). 
project out the part of the boosted operator which would give zero contribution when the trace of Eq. (3.18) is taken.) The connection between Eqs. (3.8) and (3.18) may then be made in obvious fashion if we let

$$
f=f_{e^{f}}
$$

For example, for $\tau$ as given by Eq. (3.15),

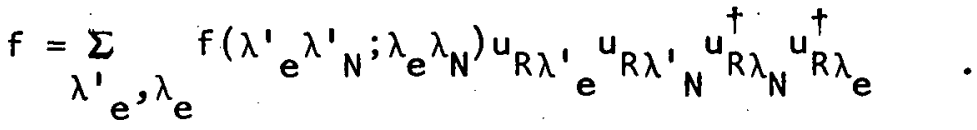

$$
\begin{aligned}
& \lambda^{\prime}{ }_{N}, \lambda_{N}
\end{aligned}
$$

We note also that $\Sigma$ is left unchanged in the boosting process and therefore may be found from either Eq. (2.2) or (3.8).

The helicity formalism, as we have outlined it here, may be used to find correlations in the c.m. frame only. Our development of the one photon exchange approximation, however, was made in the Breit frame. Thus it is necessary to relate the correlations found in the c.m. frame to those found in the Breit frame. The relation may be expressed most easily in terms of the rest frame density matrices. The covariant density matrix as given in Eq. (3.10) is a scalar in the sense that, for spin s,

$$
\rho^{\prime}=\Lambda \rho \Lambda^{-1},
$$

where $\Lambda$ is the $(0, s) \oplus(s, 0)$ representation of the Lorentz transformation. The relation between the rest frame density matrices, as found by using Eq. $(3.12)$, is

$$
\rho^{\prime}(0) \equiv\left\lceil B^{-1}(\underset{\sim}{K}) \wedge B(\mathcal{\sim})\right\rceil \rho^{(0)}\left[R^{-1}(\mathcal{L}) \Lambda^{-1} B(\underset{\sim}{K})\right],
$$

where $K_{\mu}=a_{\mu \nu} P_{\nu}$ and the $a_{\mu \nu}$ are the transformation coefficients associated with $\Lambda$. The operator in the square brackets may be shown to be rotation operators and are called Wigner rotations. In general, these rotations depend in a complicated fashion on $\Lambda$ and $\underset{\sim}{P}$, but take on fairly simple forms when $\Lambda$ is a rotation operator or a pure Lorentz transformation. When $\Lambda$ is a rotation operator, the wigner rotation may easily be shown to be just the rotation operator $\Lambda$, since

$$
\Lambda(\underset{\sim}{s} \cdot P) \cdot \Lambda^{-1}=\underline{\sim} \cdot \underline{\sim}
$$


When $\Lambda$ is a pure Lorentz transformation, the wigner rotation is most simply expressed in terms of the velocity vector $\beta$. We may give $\Lambda$ in terms of $\mathcal{B}$ as

$$
\Lambda=\exp (-s \underset{\sim}{\alpha} \cdot \hat{\sim} \operatorname{arctanh} \beta),
$$

and the transformation coefficients as

$$
\begin{aligned}
& a_{i j}=\delta_{i j}+(\eta-1) \hat{\beta}_{i} \hat{\beta}_{j}, \\
& a_{i 4}=-a_{4 i}=i \eta \beta_{i}, \\
& a_{44}=\eta,
\end{aligned}
$$

where $n=\left(1-\beta^{2}\right)^{-\frac{1}{2}}$. The parameter for the wigner rotation, which we denote by $w$, may be most easily determined by simply expanding the operators and reducing their product for the $\left(0, \frac{1}{2}\right) \oplus\left(\frac{1}{2}, 0\right)$ representation. For the $\left(0, \frac{1}{2}\right) \oplus\left(\frac{1}{2}, 0\right)$ representation, the expanded form of $\Lambda$ is given by

$$
\Lambda=[2(\eta+1)]^{-\frac{1}{2}}[\eta+1-\eta \underset{\sim}{\alpha} \cdot k] \ldots
$$

The expanded form of the boost operator is given by

where $\quad \gamma=\left[(P / M)^{2}+1\right]^{-\frac{1}{2}}$.

$$
B(P)=[2(\gamma+1)]^{-\frac{1}{2}}[\gamma+1+\underset{\sim}{\alpha} \cdot(\underset{\sim}{P} / M)],
$$

After carrying out the algebraic steps we find that the wigner rotation operator is given by

$$
\begin{aligned}
Q= & {[2 M(\eta+1)(\gamma+1)(M+M \eta \gamma-\eta \underset{\sim}{\beta} \cdot P)]^{-\frac{1}{2}} } \\
& \{[M(\eta+1)(\gamma+1)-\eta \beta \cdot P]-i \eta(\beta \times P) \cdot \underset{\sim}{\alpha}\}
\end{aligned}
$$

From this expression, it is easily shown that it is appropriate to write

$$
\operatorname{Cos}\left(\frac{1}{2} w\right)=[2 M(\eta+1)(\gamma+1)(M+M \eta \gamma-\eta \mathbb{B} \cdot P)]^{-\frac{1}{2}}[M(\eta+1)(\gamma+1)-\eta B \cdot P],(3.27 a)
$$

and

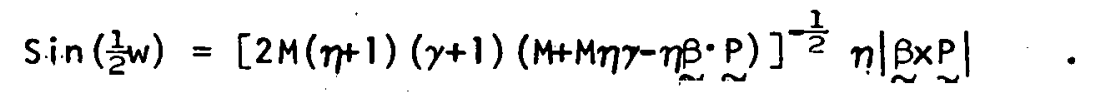

If we take $\underset{\sim}{\hat{W}}$ to be a unit vector in the direction of $(\underset{R \times P}{\mathcal{P}})$, then we may write

$$
R=\cos \left(\frac{1}{2} w\right)-i \sin \left(\frac{1}{2} w\right) \underset{\sim}{w} \cdot g=\exp \left(-\frac{1}{2} i w \cdot d\right),
$$

from which it can be seen that the wigner rotation is a rotation about $\underset{\sim}{\hat{w}}$ 
in the right-hand sense through an angle $-w$. This determines the parameter $\underset{\sim}{w}$ in general, since the reduction of the product of two general exponential forms like $\Lambda$ and $B$ depends on the commutation properties of the spin matrices, which are the same for all spin values. (This has beenpointed out previously in connection with products of general forms of $\Lambda$ by Weaver, et al. ${ }^{13}$.)

The transformation from the c.m. frame to the Breit frame may be made only by means of a pure Lorentz transformation. To determine the velocity vector $\underset{\sim}{\beta}$, we note that in the c.m. frame $\underset{\sim}{R}-\underline{\sim}^{\prime}+\underline{\sim} \neq \neq 0$, while in the Breit frame $\underset{\sim}{\mathbb{R}}=0$. Therefore, in terms of the transformation coefficients given in Eq. (3.23), we write

$$
0=\underset{\sim}{R}+(\eta-1) \stackrel{R}{R} \cdot \stackrel{R}{R}-2 \eta R^{E},
$$

where $E$ is the $c . m$. energy of the nucleus. The velocity vector given by

$$
\dot{B}=(2 E)^{-1} \stackrel{R}{\sim}
$$

is the only vector which satisfies Eq. (3.28). The parameter $\eta$ associated with this transformation is

$$
n=\left(-\mathrm{R} \mu_{\mu}^{\mathrm{R}} / 4\right)^{-\frac{1}{2}} \mathrm{E},
$$

where $R_{\mu}=(\underset{\sim}{R}, 2 i E)$. The transformation of the momentum vectors of the initial and final states of the nucleus is easily found to be

$$
a_{i \nu} P_{\nu}=-\frac{1}{2} Q
$$

and

$$
a_{i} P^{P^{\prime}}=\frac{1}{2} Q
$$

in agreement with the definition of the Breit frame of the nucleus. This follows from the definition of $\underset{\sim}{\beta}$ in Eq. (3.29) and the use of $\underset{\sim}{R} \cdot \underset{\sim}{P}=\underset{\sim}{R} \cdot \sim^{\prime}$ $=\frac{1}{2} R \cdot R$. We may further show that the momentum transfer three-vector is left unchanged by this transformation, since in the c.m. $Q_{\mu}=(Q, 0)$ and $\underset{\sim}{Q} \cdot R=0$, which is also in agreement with the definition of the Breit frame:

The connection between the c.m. frame and Breit frame correlations may now be carried out explicitly using the wigner rotation given in Eq. (3.26) and the velocity vector as given in Eq. $(3.29)$. The Wigner 
rotations, for both the initial and final states of the nucleus and the electron, are about the normal to the scattering plane, since $\underset{\sim}{\mathbb{R}}$ and all the momentum vectors lie in the scattering plane. If we write the initial momentum of the nucleus in the c.m. frame as

$$
\stackrel{P}{\sim}=\frac{1}{2} \underset{\sim}{(R-Q)},
$$

and the final momentum of the nucleus as.

$$
\stackrel{P}{\sim}^{\prime}=\frac{1}{2}(\underset{\sim}{R+Q}),
$$

then it is clear that the rotation associated with the final nuclear state is oppositely directed to the one associated with the initial nuclear state. In addition, since the energy of the initial and final states of the nucleus are the same, the angular displacements of the rotations are the same size. Consequently, the rotation associated with the final nuclear state is the inverse of the rotation associated with the initial nuclear state. The rotations associated with the initial and final states of the electron are similarly related. Also, since the momenta of either the initial or final states of the electron and nucleus differ only in sign, the rotations associated with these states are oppositely directed. However, the angular displacements associated with either the electron states or with the nuclear states are not of the same size, and no other simple relation exists between them. 


\section{DISCUSSION OF SOME OF THE OBSERVABLES}

IN THE ELASTIC SCATTERING PROCESS

Before discussing any specific examples of the quantities which may be observed, we need to specify how the correlations are to be found in the one photon exchange approximation. We will also examine some general considerations which apply for all the correlations.

The boosted form of Eq. (2.2) as discussed in Chapter 111 is also useful for discussing the correlations which may be observed in the elastic electron-nucleus scattering process. "For the covariant description of the scattering process, the matrix $T$ in Eq. (2.2) is given by

$$
T=J_{e} J_{N_{\mu}},
$$

where, as usual in quantum electrodynamics, we take the electron part to be given by

$$
\mathrm{J}_{\mathrm{e}_{\mu}}=i \gamma_{\mu}
$$

The matrix $\gamma_{\mu}$ is the usual Dirac matrix which is equal to our $\gamma_{3, \mu}$. As we have pointed out previously and as given in Table 7 in Chapter II, this term is Hermitian and invariant under each reflection, $P, C$, and $T$. This term also gives rise to a conserved current, as may be shown directly from either Eq. (2.45), the spinor equation, or from Eqs. (5.45), the boosted current form. The nuclear part of Eq. (4.1) has been discussed in Chapter 11 and may be taken as given. In order to make use of a boosted form, such as is given in Eq. (3.8), we make the identification

$$
\tau_{e}=J_{e}, \quad \text { and } \tau_{N}=J_{N} \text {. }
$$

Thus we may factorize Eq. (2.2), as was done in Eq. (3.16), and write

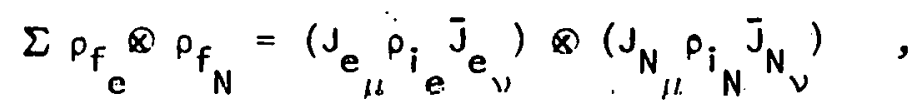

where $\bar{J}_{\mu}=\beta J_{\mu}^{\dagger} \beta$ (the dagger denotes the matrix Hermitian conjugate): The boosted form of this equation follows immediately with: the recognition that 


$$
j_{\mu}=\frac{1}{2}(1+B) B^{-1}\left(P^{\prime}\right) J_{\mu} B(P) \frac{1}{2}(1+B),
$$

and the buusled furm may be written as

$$
\sum \rho_{f}^{(0)} \otimes \rho_{f_{N}}^{(0)}=\left(j_{e} \rho_{\mu} \rho_{i}^{(0)} j^{\dagger} e_{\nu}\right) \otimes\left(j_{N} \rho_{\mu}^{(0)} j_{N}^{+} j_{N}\right)
$$

The boosted expressions given by both Eq. (3.8) and Eq. (4.5) involve the. $2(2 s+1)$ - square matrices $\underset{\sim}{s}$. As pointed out in connection with Eq. (3.11), this simply implies a certain normalization of the density matrices, but otherwise the functional forms are no different than if the expansions of Eqs. (3.8) and (4.5) were made in terms of the $(2 \mathrm{~s}+1)$ - square matrices $\underset{\sim}{s}$. Therefore, we are free to work with matrices of either dimensionality, as long as we allow properly for the normalization of the density matrices.

Although we have introduced the boosted current and used the rest frame density matrices in Eq. (4.5), this equation is still covariant if we use a general boosted form for the currents. The covariant current terms transform as

$$
J^{\prime}{ }_{\mu}=a_{\mu \nu} \Lambda J_{\nu} \Lambda^{-1}
$$

where $\Lambda$ is the $(0, s) \oplus(s, 0)$ representation of the Lorentz group. Using this transformation property, the relation between the boosted form of the transformed current and the original covariant current is found to be

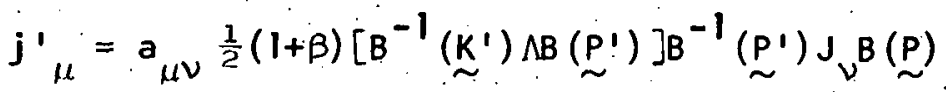

$$
\begin{aligned}
& {\left[\mathrm{B}^{-1} \underset{\sim}{(P)} \Lambda^{-1} \mathrm{~B}(\underset{\sim}{K})\right] \frac{1}{2}(1+\beta),}
\end{aligned}
$$

where. $K^{\prime}{ }_{\mu}=a_{\mu \nu}{ }^{\prime \prime}{ }_{\nu}$,

and $\quad K_{\mu}=a_{\mu \nu} P_{\nu}$,

and the transformation coefficients are those associated with $\Lambda$. The quantities in the square brackets are recognizable as the wigner rotations discussed in connection with Eq. (3.21). The rotations and $\beta$ commute in general so that Eq. (4.6) may be written as

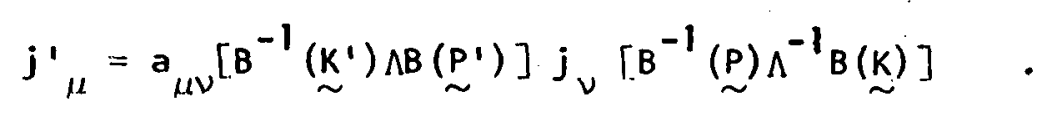


(The transformation of the boosted current is not in general a rotation unless $\Lambda$ itself is a rotation operator.) The transformation properties of the density matrices as given in Eq. (3.21) and the boosted current are sufficient to ensure that Eq. (4.5) is covariant. Our considerations become noncovariant only when we. choose to work in a specific reference frame.

We shall choose here to make our considerations in the Breit frame. The boosted nuclear current may then be taken to be given by Eqs. (2.66) and $(2.67)$ if we choose the z-axis to be defined by the direction of the incident momentum of the nucleus. The boosted form of the electron current is given by Eq. $(5.45)$ as

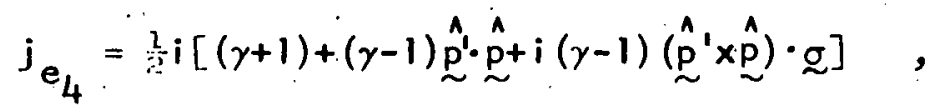

and

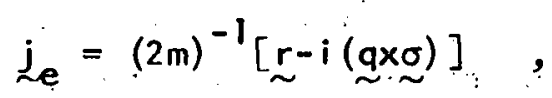

where $\underset{\sim}{r}=\mathfrak{p}^{\prime}+\underline{p}$ and $q=\underline{p}^{\prime}-\underline{q}$. We note that, from conservation of momentum, $\dot{q}=-\underline{0}$

We shall also choose to work with the $(2 s+1)$ - square matrices. The expansion of the spin "sensity matrix" is then given by

$$
\rho^{\left(0^{\prime}\right)}=(2 s+1)^{-1} \mathrm{~L}_{L, M} \rho_{L M}{ }^{\top}{ }^{\dagger},
$$

where the $T_{L M}$ is the set of matrices discussed in Appendix $A$, and the $P_{L M}$ are statistical tensors of rank $L$ and component $M$. In order for the density matrix to be Hermitian (in the matrix sense) we require that

$$
\theta_{L M}^{*}=(-1)^{M} \theta_{L,-M},
$$

since $T_{L M}^{+}=(-1)^{M} T_{L,-M}$. The factor of $(2 s+1)$ is included so that $\operatorname{Tr} \rho=1$, as follows from $Q_{00}=1$, and the trace property of $T_{L M}$ given by Eq:

(5.12). The statistical tensors are given in terms of.the density matrix and the TLM by

$$
\left.Q_{\mathrm{L} M}=\operatorname{Tr}\left[\rho^{(0)}\right)_{L M}\right]
$$

which follows from Eqs. (5.12) and (4.8). 
The normalization factor $\Sigma$ may now be found for the one photon exchange approximation by taking the traçe of Eq. (4.5). This results in

$$
\Sigma=\sigma_{\mathbf{e}} \sigma_{\mu \nu}
$$

where $\quad \sigma_{\mu \nu}=\operatorname{Tr}\left(\mathbf{j}_{\mu} \rho_{i}^{(0)} \mathbf{j}_{\nu}^{+}\right)$.

The quantity given by Eq. $(4.12)$ is a Lorentz tensor for the general boosted current. The reality of the components of this tensor is related to the symmetry of $i$ ts indices. Taking the complex conjugate gives

$$
\sigma_{\mu \nu}^{*}=\operatorname{Tr}\left(j_{\nu} \rho_{i}^{(0)} j_{\mu}^{+}\right)=\sigma_{\nu \mu},
$$

which follows from the transposition of the trace and the Hermiticity of the density matrix: Thus, the symmetric part of $\sigma_{\mu \nu}$, which we denote by $\sigma_{\mu \nu}^{(S)}=\frac{1}{\partial}\left(\sigma_{\mu \nu}+\sigma_{\nu \mu}\right)$, is real, and the antisymmetric part, which we denote by $\sigma_{\mu \nu}(A)=\frac{1}{r}\left(\sigma_{\mu \nu}-\sigma_{\nu \mu}\right)$, is pure imaginary. From Eqs. $(4.11)$ and $(4.13)$ it is easy to show that $\Sigma$ is real. The symmetric and antisymmetric parts of $\sigma_{\mu \nu}$ also differ in their dependence on the rank of the statistical tensors as we shall demonstrate later. If the trace of Eq. (4.5) is expanded in. terms of the matrix elements of the electronic and nuclear currents, it becomes evident that $\Sigma$ is just the cross section (within some normalization) for the scattering of a beam of polarized electrons on an oriented nuclear target to some final configuration in which the orientation is unobserved. Therefore, any asymmetry in this cross section is given by

$$
\text { As }=\frac{\Sigma_{L}-\Sigma_{R}}{\Sigma_{L}+\Sigma_{R}} \text {, }
$$

where the subscripts $L$ and $R$ are used here to denote two different initial orientations of the beam and the target.

The polarization vector of the final electron states may be found from Eq. (4.5) by multiplying both sides by $\mathbb{Z}$ and taking the trace. The result is

$$
\Sigma{\underset{\sim}{P}}_{\mathbf{e}}^{(f)}=\left(Q_{\mathbf{e}}\right)_{\mu \nu} \sigma_{\mu \nu},
$$


where

$$
\left({\underset{\sim e}{e}}_{\mu \nu}^{\boldsymbol{P}}\right)=\operatorname{Tr}\left(\sigma_{\sim}^{j} e_{\mu} \rho_{i}^{(0)} j_{e_{\nu}^{+}}^{+}\right)
$$

and $\sigma_{N_{\mu N}}$ is given by Eq. (4.12). The quantity $\left(\mathbb{P}_{e}\right)_{\mu \nu}$ is a Lorentz tensor on the indices $\mu \nu$ and a three-vector on the vector indices of $g$. Under a general Lorentz transformation, the three-vector part of this quantity is rotated according to the wigner rotation associated with the final state. The symmetry of the $\mu \nu$ indices of this quantity also is related to its reality. Taking the complex conjugate gives

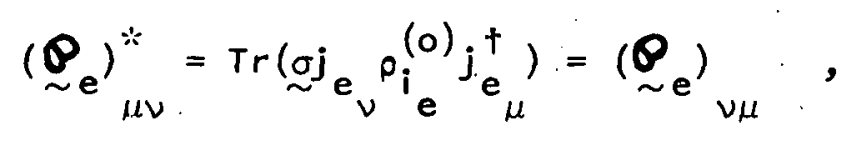

which follows in a manner similar to that used with Eq. (4.13) when we include the Hermiticity of the matrices $\sigma$. Thus the symmetric part of

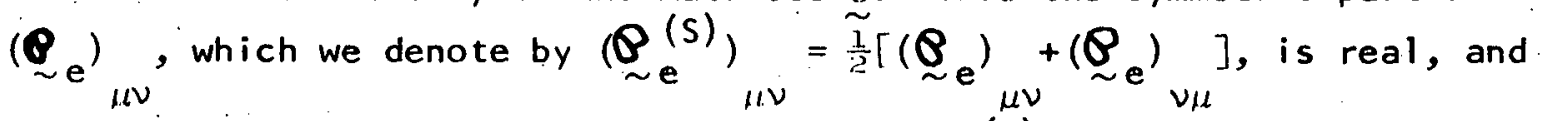
the antisymmetric part, which we denote by $(\underset{\sim}{\boldsymbol{P}}(A))=\frac{2}{2}\left[\left({\underset{\sim}{\mathrm{e}}}_{\mu}\right)-\left({\underset{\sim}{\mathrm{e}}}_{\mu \nu}\right)\right]$, is pure imaginary. As with $\sigma_{\mu \nu}$, the symmetric and antisymmetric parts differ in their dependence on the rank of the initial statistical tensor. Similar considerations could be made for the polarization tensors which describe the final nuclear state. However, the determination of the polarization of the final state of even the nucleons is difficult experimentally, so we shall confine our attention to correlations which are involved with asymmetries in the cross section or the polarization of the final electron state.

A. Unoriented Target and Beam

For the situation in which the target and the beam are unoriented the density matrices of the nucleus and the electron are given by

$$
\rho_{i}^{(0)}=(2 s+1)^{-1}
$$

and

$$
\rho_{i}^{(0)}=\frac{1}{2},
$$

respectively. The contribution of the electron is given by

$$
\sigma_{e_{44}}=\frac{1}{2}\left[\left(\gamma^{2}+1\right)+\left(\gamma^{2}-1\right) \hat{Q^{1}} \cdot \hat{Q}\right]=\left(4 m^{2}\right)^{-1}\left(4 m^{2}+r \cdot r\right),
$$




$$
\begin{aligned}
& \sigma_{e_{i 4}}^{(A)}=-i(2 m)^{-1} \gamma r_{i}, \\
& \sigma_{e_{i j}}^{(s)}=\left(4 m^{2}\right)^{-1}\left(r_{i} r_{j}-q_{i} q_{j}+\delta_{i j} q \cdot q\right)
\end{aligned}
$$

and all the other parts of $\sigma_{e_{\mu \nu}}$ are zero. At this point, these expressions apply equally well to the muon since we have not taken the ultrarelativistic limit for the electron. ( $1 \mathrm{t}$. should also be noted that $\sigma_{44}^{(s)}=\sigma_{44}$.) The contribution of the nucleus to $\Sigma$, as found from Eqs. (2.66) and (2.67), is given by

$$
\begin{aligned}
& \sigma_{N_{44}}=\sum_{\text {even } L}\left(\Gamma_{L}^{(c)}\right)^{2}, \\
& \sigma_{N_{i j}}^{(s)}=\left[\sum_{\text {odd } L}\left(\Gamma_{L}^{(m)}\right)^{2}+\sum_{\text {even } L}\left(\Gamma_{L}^{e)}\right)^{2}\right] \frac{1}{2}\left(\delta_{i 1} \delta_{j 1}+\delta_{i 2} \delta_{j 2}\right),
\end{aligned}
$$

and all other parts of $\sigma_{N_{\mu \nu}}$ are zero. Eqs. (4.19) follow directly from the form of the components of the boosted current with the use of the trace relation given in Eq. (5.12). Since the z-axis was chosen to be in the $-\hat{0}$ direction, the function of the Kronecker deltas which appears in Eq. (4.19b) may be written as

$$
\delta_{i 1} \delta_{j 1}+\delta_{i 2} \delta_{j 2}=\delta_{i j}-\hat{Q}_{i} \hat{Q}_{j}
$$

From this it follows that

$$
\begin{aligned}
\Sigma= & \left(4 m^{2}\right)^{-1}\left\{\left(4 m^{2}+\underset{\sim}{r} \underset{\sim}{r}\right) e_{\text {even } L}\left(\Gamma_{L}^{(c)}\right)^{2}\right. \\
& \left.+(\underset{\sim}{r} \underset{\sim}{r+2 q} \underset{\sim}{q})\left[\underset{\text { odd } L}{\Sigma}\left(\Gamma_{L}^{(m)}\right)^{2}+\sum_{\text {even } L}\left(\Gamma_{L}^{(e)}\right)^{2}\right]\right\} .
\end{aligned}
$$

Thus $\Sigma$ has no asymmetries and the dependence on $\Gamma_{L}^{(m)}$ and $\Gamma_{L}^{(e)}$ is impossible to separate, a result which is not surprising and was apparent from the form of Eq. (4.19b). Therefore, it is also apparent that nothing is to be gained, as far as an understanding of the time-reversal noninvariant part is concerned, from observing the polarization of the electron. We note, however, that the contribution of the electron to the final polarization is given by 


$$
\begin{aligned}
& \left(Q_{e_{\ell}(s)}^{(s)}=-(4 m)^{-1}\left\{( \gamma - 1 ) \left[q _ { \ell } \left({\left.\stackrel{p}{p^{\prime}} \times \hat{p}\right)_{i}}_{i}+\left(\hat{p}^{\prime} \times \hat{p}\right)_{\ell}{ }^{r} i\right.\right.\right.\right. \\
& \left.+\left[\gamma+1+(\gamma-1) \hat{p}^{\prime} \cdot \hat{p}\right] \epsilon_{\ell, i j} q_{j}\right\},
\end{aligned}
$$

and

$$
\left(\theta_{e_{l}}^{(A)}\right)=i\left(4 m^{2}\right)^{-1} \epsilon_{i j k} q_{k}\left(r_{\ell}+q_{l}\right)
$$

From the expression for the final electron polarization, Eq. (4.15), using Eqs. (4.19) and (4.21), we find that the polarization is zero regardless of whether or not the reaction is time-reversal invariant.

\section{B. Unoriented Target--Polarized Beam}

When the beam of electrons initially has some polarization, the density matrix of the beam is given by

$$
\rho_{i}^{(0)}=\frac{1}{2}\left(1+{\underset{\sim}{Q}}_{\mathrm{e}}^{(i)} \cdot \sigma\right)
$$

where $\underset{\sim}{\mathbb{e}^{(i)}}$ is the initial polarization vector of the beam. In general the components of $\sigma_{e}$ are now nonzero, but the parts given in Eqs. (4.18) are unchanged. Thus the division into symmetric and antisymmetric parts has also led to a division according to the rank of the initial polarization of the electron. The parts of $\sigma_{e_{\mu \nu}}$ not given in Eqs. (4.18) are given by

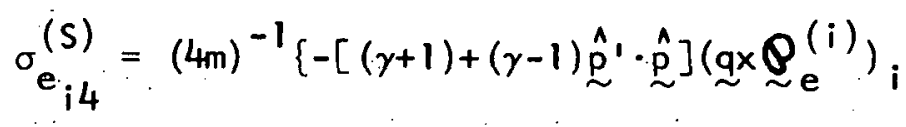

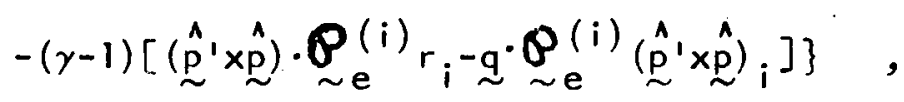

$$
\begin{aligned}
& \left.\sigma_{e_{i j}}^{(A)}=-i\left(4 m^{2}\right)^{-1}\left\{\epsilon_{i j k} q_{k}\left(\underset{\sim}{q} \cdot \underset{\sim}{\theta_{e}^{(i)}}\right)-\epsilon_{i j k}[\underset{\sim}{\operatorname{rx}} \underset{\sim}{\operatorname{qx}} \underset{\sim}{\underset{\sim}{\rho}}(i))\right]_{k}\right\}
\end{aligned}
$$

However, the parts of $\sigma_{N_{\mu \nu}}$ which are contracted with the parts of $\sigma_{e_{\mu \nu}}$ in Eqs. (4.22) are still zero and $\Sigma$ is still given by Eq. (4.20).

The other components of $\left(\mathbb{Q}_{\mathrm{e}}\right)_{\mu \nu}$ are also nonzero in general. These components are given by

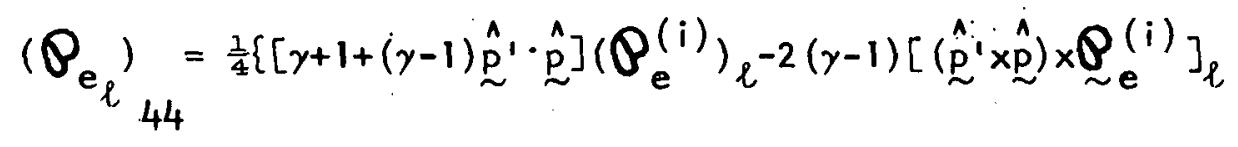




$$
\begin{aligned}
& \left.+(\gamma-1)^{2} \epsilon_{\ell m n}\left(\underline{\sim}^{\prime} \times \hat{p}\right){ }_{m}\left[\left(\hat{p}^{\prime} \times \underset{\sim}{\hat{p}}\right) \times \underset{\sim}{P} e^{(i)}\right]_{n}\right\},
\end{aligned}
$$

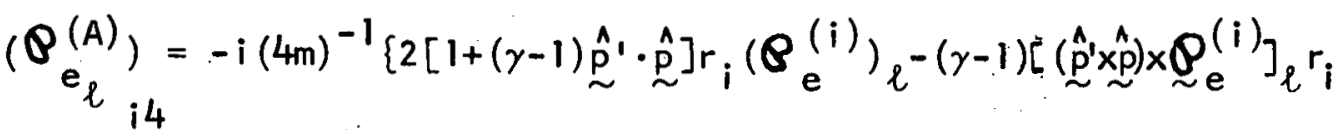

$$
\begin{aligned}
& +[\gamma+1+(\gamma-1) \underset{\sim}{\hat{p}} \cdot \cdot \underset{\sim}{\hat{p}}]\left[q_{\ell}\left(\nabla_{e}^{(i)}\right)_{i}-\delta_{\ell i} q^{q} \cdot{\underset{\sim}{e}}_{e}^{(i)}\right]
\end{aligned}
$$

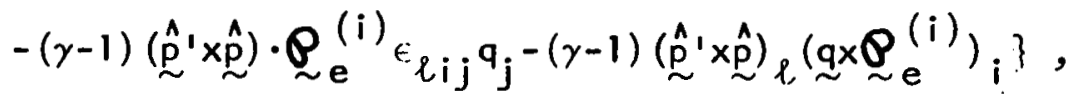

$$
\begin{aligned}
& \left(\theta_{e_{l}}^{(s)}\right)=\left(4 m^{2}\right)^{-1}\left\{\left(r_{i} r_{j}+q_{i} q_{j}-\underset{\sim}{q} \cdot q_{i j} \kappa_{i j}\right)\left(\nabla_{e}^{(i)}\right)_{l}\right. \\
& +\left[r_{i}\left(\nabla_{e}^{(i)}\right)_{j}+r_{j}\left(\nabla_{e}^{(i)}\right)_{i}\right] q_{l}-\left(r_{i} \delta_{j l}+r_{j} \hat{n}_{i l}\right) \underset{\sim}{q} \underbrace{(i)}_{e}
\end{aligned}
$$

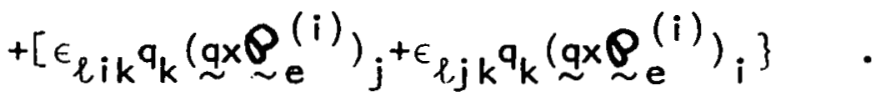

The final polarization of the electron is therefore given by

$$
\begin{aligned}
& \Sigma \mathbb{\sim}_{e}^{(f)}=\frac{1}{4}\left\{\left[\gamma+1+(\gamma-1) \hat{p}^{\prime} \cdot \hat{p}\right] P_{e}^{(i)}-2(\gamma-1)\left(\hat{p}^{\prime} \times \hat{p}\right) \times \mathbb{\sim}_{e}^{(i)}\right. \\
& \left.+(\gamma-1)^{2}(\underset{\sim}{\hat{p}} \times \underset{\sim}{\hat{p}}) \times\left[\left(\hat{\sim}_{\sim}^{\prime} \times \hat{p}\right) \times \underset{\sim}{\mathbb{P}^{(i)}}\right]\right\}\left[\underset{\text { even } L}{\sum}\left(\Gamma_{L}^{(c)}\right)^{2}\right] \\
& +\left(4 m^{2}\right)^{-1}\left\{\left(r \cdot L^{-2} q \cdot \underset{\sim}{q}\right) \underset{\sim}{P} e^{(i)}+2\left(\underset{\sim}{r} \cdot \underset{\sim}{P} e^{(i)}\right) \underset{\sim}{q-2}\left(\underset{\sim}{q} \cdot \underset{\sim}{\stackrel{P}{P}} e^{(i)}\right) r\right.
\end{aligned}
$$

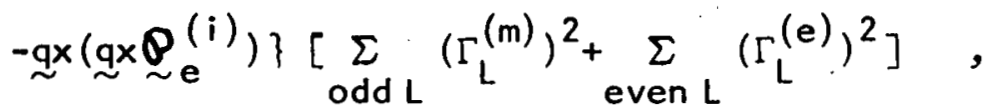

so that the final electron beam actually has a nonzero polarization. However, as for the case when the initial electron beam has no polarization, the dependence on $\Gamma_{L}^{(m)}$ and $\Gamma_{L}^{(e)}$ cannot be separated and no information is available about the time-reversal noninvariant terms when the nucleus has no orientation.

\section{Oriented Target--Unpolarized Beam}

When the nuclear target has some orientation, the density matrix describing the target is given by

$$
\rho_{i_{N}}^{(0)}=(2 s+1)^{-1} \sum_{L, M} \Theta_{L M} T_{L M}^{\dagger} \text {, }
$$

in agreement with Eq. (4.8). Thus in order to evaluate $\sigma_{N_{\mu \nu}}$ we must first, 
determine the trace

$$
\operatorname{Tr}\left(T_{L M^{\prime}} T_{L \prime M}^{\dagger} T_{L \prime \prime M}^{\prime \prime \prime}\right)
$$

As the first step, we write the matrix element of the $T_{L M}$ as

$$
\left(T_{L M^{\prime}}\right)_{\lambda \lambda^{\prime}}=(2 L+1) C\left(s L s ; \lambda^{\prime} M \lambda\right),
$$

where we have used the symmetry of the C's to rewrite Eq. (5.11). Thus the trace may be written as

$$
\begin{aligned}
& \operatorname{Tr}\left(T_{L M^{\prime}} T_{L^{\prime} M^{\prime}}^{\dagger} T_{L^{\prime \prime} M^{\prime \prime}}^{\dagger}\right)=\left[(2 L+1)\left(2 L^{\prime}+1\right)\left(2 L^{\prime \prime}+1\right)\right]^{\frac{1}{2}} \\
& \sum \sum_{\lambda, \lambda^{\prime}, \lambda^{\prime \prime}} \mathrm{C}\left(s L s ; \lambda^{\prime} M \lambda\right) C\left(s L^{\prime} s ; \lambda^{\prime} M^{\prime} \lambda^{\prime \prime}\right) C\left(s L^{\prime \prime} s ; \lambda^{\prime \prime} M^{\prime \prime} \lambda\right)
\end{aligned}
$$

Again, using the symmetry of the C's, we may write

$$
C\left(s L s ; \lambda^{\prime} M \lambda\right) C\left(s^{\prime \prime \prime} s ; \lambda^{\prime \prime} M^{\prime \prime} \lambda\right)=(-1)^{M^{\prime \prime}} C\left(s L s ; \lambda^{\prime} M \lambda\right) C\left(s L^{\prime \prime} s ; \lambda,-M^{\prime \prime}, \lambda^{\prime \prime}\right) \text {. }
$$

The sum over $\lambda$ in Eq. (4.26) may then be carried out with the result

$$
\begin{gathered}
\sum c\left(s L s ; \lambda^{\prime} M \lambda\right) C\left(s L^{\prime \prime} s ; \lambda^{\prime \prime} M^{\prime \prime} \lambda\right)=(-1) M^{M^{\prime \prime}} c\left(s \lambda s ; \lambda^{\prime}, M, \lambda^{\prime}+M\right) \\
C\left(s L^{\prime \prime} s ; \lambda^{\prime}+M,-M^{\prime \prime}, \lambda^{\prime \prime}\right) .
\end{gathered}
$$

The product of the C's on the right in Eq. (4.28) may be rewritten in terms of Racah coefficients [c.f., Eq. (6.4a) Rose ${ }^{26}$ ] so that

$$
\begin{aligned}
& \sum c\left(s L s ; \lambda^{\prime} M \lambda\right) c\left(s L^{\prime \prime} s ; \lambda^{\prime \prime} M^{\prime \prime} \lambda\right)=(-1)^{M^{\prime \prime}} \sum_{\lambda}[(2 j+1)(2 s+1)]^{\frac{1}{2}} \\
& \quad W\left(s L s L^{\prime \prime} ; s j\right) C\left(L L^{\prime \prime} j ; M,-M^{\prime \prime}\right) C\left(s j s ; \lambda^{\prime}, M-M^{\prime \prime}, \lambda^{\prime \prime}\right),
\end{aligned}
$$

where $W\left(s L s L^{\prime \prime} ; s j\right)$ is a Racah coefficient. Now the sum over $\lambda^{\prime \prime}$ in Eq. (4.26), using (4.29), gives

$$
\begin{aligned}
\underset{\lambda, \lambda^{\prime \prime}}{\sum C\left(s L s ; \lambda^{\prime} M \lambda\right) C\left(s L^{\prime \prime} s ; \lambda^{\prime \prime} M^{\prime \prime} \lambda\right) C\left(s L^{\prime} s ; \lambda^{\prime} M^{\prime} \lambda^{\prime \prime}\right)} \\
=(-1)^{M^{\prime \prime}} \sum_{j}[(2 j+1)(2 s+1)]^{\frac{1}{2}} W\left(s L s L^{\prime \prime} ; s j\right) C\left(L L^{\prime \prime} j ; M,-M^{\prime \prime}\right) \\
\left.\quad \underset{\lambda !^{\prime \prime}}{\sum} c\left(s j s ; \lambda^{\prime}, M-M^{\prime \prime}, \lambda^{\prime \prime}\right) C\left(s L^{\prime} s ; \lambda^{\prime}, M^{\prime}, \lambda^{\prime \prime}\right)\right],
\end{aligned}
$$

which is zero unless $\lambda^{\prime \prime}=\lambda^{\prime}+M-M^{\prime \prime}=\lambda^{\prime}+M^{\prime}$, so that $M^{\prime}=M-M^{\prime \prime}$. In that case the sum on $\lambda^{\prime \prime}$ on the right in Eq. (4.30) may be replaced by 


$$
C\left(s j s ; \lambda^{\prime}, M^{\prime}, \lambda^{\prime}+M^{\prime}\right) C\left(s L^{\prime} s ; \lambda^{\prime}, M^{\prime}, \lambda^{\prime}+M^{\prime}\right)
$$

The final sum on $\lambda^{\prime}$ may be carried out using the symmetry properties and the orthogonality of the C's with the result

$$
\begin{gathered}
\operatorname{Tr}\left(T L M^{\top} L^{\prime} M^{\prime} T_{L^{\prime \prime} M^{\prime \prime}}^{\dagger}\right)=(2 s+1)^{\frac{3}{2}}\left[(2 L+1)\left(2 L^{\prime \prime}+1\right)\right]^{\frac{1}{3}} \\
W\left(s L s L^{\prime \prime} ; s L^{\prime}\right) C\left(L L^{\prime \prime} L^{\prime} ; M,-M^{\prime \prime}, M^{\prime}\right)
\end{gathered}
$$

The vector part of the nuclear current given in Eq. (2.67) may be written in component form as

$$
\begin{aligned}
j_{i}= & \sum_{\text {odd } L} \Gamma_{L}^{(m)}\left(-\frac{1}{2}\right)\left[\delta_{i+} T_{L,-1}+\delta_{i-}{ }^{\top} L,+1\right. \\
& +i \sum_{\text {even } L} \Gamma_{L}^{(e)}\left(\frac{1}{2}\right)\left[\delta_{i+} T_{L,-1}-\delta_{i}-T_{L,+1}\right],
\end{aligned}
$$

where $\quad \delta_{i \pm}={\underset{\sim}{e}}_{i} \cdot e_{ \pm}^{e}$ and $\underset{\sim \pm}{e}={\underset{\sim x}{e} \pm}_{\sim}^{e}$.

The components of $\sigma_{N_{\mu \nu}}$ then follow from Eqs. (2.66) and (4.32) with the use of the trace relation $(4.31)$ as

$$
\begin{aligned}
& \begin{array}{c}
\sigma_{N_{44}}=\underset{\substack{\text { even } L \\
\text { even } L^{\prime \prime} \\
L^{\prime}}}{\sum} \Gamma_{L^{\prime}}^{(c)} \Gamma_{L^{\prime \prime}}^{(c)}\left[(2 s+1)(2 L+1)\left(2 L^{\prime \prime}+1\right)\right]^{\frac{1}{2}} Q_{L^{\prime} 0} \\
\quad W\left(s L s L^{\prime \prime} ; s L^{\prime}\right) C\left(L L^{\prime \prime} L^{\prime} ; 0,0\right)
\end{array} \\
& \sigma_{N_{i 4}}^{(s)}=\underset{\substack{\text { even } L \\
\text { odd } L^{\prime \prime}}}{\sum}\left\{\Gamma_{L}^{(c)} \Gamma_{L^{\prime \prime}}^{(m)} \cdot\left[(2 s+1)(2 L+1)\left(2 L^{\prime \prime}+1\right)\right]^{\frac{1}{2}} W\left(s L s L^{\prime \prime} ; s L^{\prime}\right)\right. \\
& \left.c\left(L L^{\prime \prime} L ; 0,-1\right)(i / 4)\left[1+(-1)^{L^{\prime}+1}\right]\left[\delta_{i+} Q_{L^{\prime},-1}+\delta_{i}-Q_{L^{\prime},+1}\right]\right\} \\
& +\sum_{\text {even } L}\left\{\Gamma_{L}^{(c)} \Gamma_{L^{\prime \prime}}^{(e)}\left[(2 s+1)(2 L+1)\left(2 L^{\prime \prime}+1\right)\right]^{\frac{1}{2}} W\left(s L s L^{\prime \prime} ; s L^{\prime}\right)\right. \\
& \text { even L" } \\
& L^{\prime} \\
& \left.c\left(L^{\prime \prime} L^{\prime} ; 0,-1\right)\left(\frac{1}{4}\right)\left[1+(-1)^{L^{\prime}}\right]\left[\delta_{i+} \otimes_{L^{\prime},-1}-\delta_{i-} \Theta_{L^{\prime},+1}\right]\right\} \text {, }
\end{aligned}
$$




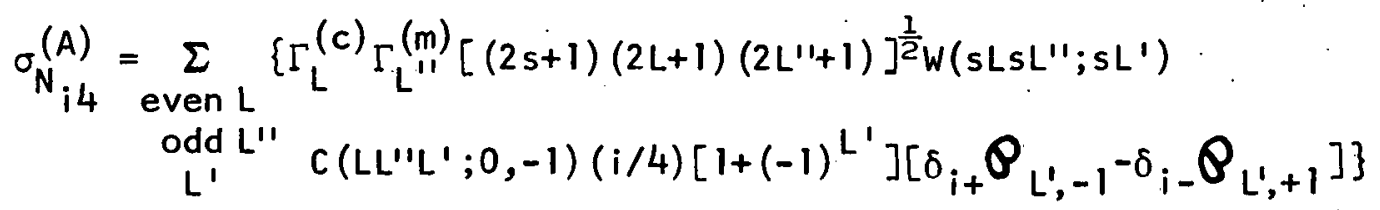

$$
\begin{aligned}
& +\sum_{\text {even } L}\left\{\Gamma_{L}^{(c)} \Gamma_{L^{\prime \prime}}^{(e)}\left[(2 s+1)(2 L+1)\left(2 L^{\prime \prime}+1\right)\right]^{\frac{1}{2}} W\left(s L s L^{\prime \prime} ; s L^{\prime}\right)\right. \\
& \left.L^{\prime} \quad c\left(L L^{\prime \prime} L^{\prime} ; 0,-1\right)\left(\frac{1}{4}\right)\left[1+(-1)^{L^{\prime}+1}\right]\left[\delta_{i}+\theta_{L^{\prime},-1}+\delta_{i-} \theta_{L^{\prime},+1}\right]\right\} \text {, } \\
& \sigma_{i j}^{(s)}=-\sum_{\operatorname{odd} L}\left\{\Gamma_{L}^{(m)} \Gamma_{L^{\prime \prime}}^{(m)}\left[(2 s+1)(2 L+1)\left(2 L^{\prime \prime}+1\right)\right]^{\frac{1}{2}} W\left(s L s L^{\prime \prime} ; s L^{\prime}\right)\right. \\
& \underset{L^{\prime}}{\operatorname{odd} L^{\prime \prime}}\left(\frac{1}{8}\right)\left[1+(-1)^{L^{\prime}}\right]\left[\delta_{i+} \delta_{j-}+\delta_{i-} \delta_{j+}\right) P_{L^{\prime} 0^{C}} C\left(L L^{\prime \prime} L^{\prime} ;-1,1\right) \\
& \left.\left.+\left(\delta_{i+} \delta_{j+} P_{L^{\prime},-2}+\delta_{i-} \delta_{j}-P_{L^{\prime}, 2}\right) C\left(L^{\prime \prime} L^{\prime} ;-1,-1\right)\right]\right\} \\
& \underset{\text { even } L}{-\sum}\left\{\Gamma_{L}^{(e)} \Gamma_{L^{\prime \prime}}^{(e)}\left[(2 s+1)(2 L+1)\left(2 L^{\prime \prime}+1\right)\right]^{\frac{1}{2}} W\left(s L s L^{\prime \prime} ; s L^{\prime}\right)\right. \\
& \underset{L^{\prime}}{\operatorname{even} L^{\prime \prime}}\left(\frac{1}{8}\right)\left[1+(-1)^{L^{\prime}}\right]\left[\left(\delta_{i+} \delta_{j-}+\delta_{i-} \delta_{j+}\right) P_{L^{\prime} 0} C\left(L L^{\prime \prime} L^{\prime} ;-1,1\right)\right. \\
& \left.\left.-\left(\delta_{i+} \delta_{j+} \nabla_{L^{\prime},-2}+\delta_{i-} \delta_{j}-P_{L^{\prime}, 2}\right) c\left(L^{\prime \prime} L^{\prime} ;-1,-1\right)\right]\right\} \\
& +i \underset{\text { odd } L}{\sum}\left\{\Gamma_{L}^{(m)} \Gamma_{L^{\prime \prime}}^{(e)}\left[(2 s+1)(2 L+1)\left(2 L^{\prime \prime}+1\right) \cdot\right]^{\frac{1}{2}} W\left(s L s L^{\prime \prime} ; s L^{\prime}\right)\right. \\
& \underset{L^{\prime}}{\operatorname{even} L^{\prime \prime}}\left(\frac{1}{4}\right)\left[1+(-1)^{L^{\prime}+1}\right]\left(\delta_{i+} \delta_{j+} \nabla_{L^{\prime},-2}-\delta_{i-} \delta_{j}-P_{L^{\prime}, 2}\right) \\
& \left.C\left(L^{\prime} L^{\prime} L^{\prime} ;-1,-1\right)\right\} \text {, }
\end{aligned}
$$

$$
\begin{aligned}
& \sigma_{i j}^{(A)}=\underset{\text { odd } L}{-\sum_{L}}\left\{\Gamma_{L}^{(m)} \Gamma_{L ! !}^{(m)}\left[(2 s+1)(2 L+1)\left(2 L^{\prime \prime}+1\right)\right]^{\frac{1}{2}} W\left(s L s L^{\prime \prime} ; s L^{\prime}\right)\right. \\
& \operatorname{Ldd}^{\prime} L^{\prime \prime}\left(\frac{1}{8}\right)\left[1+(-1)^{L^{\prime}+1}\right]\left[\delta_{i+} \delta_{j-}-\delta_{i-} \delta_{j+}\right) Q_{L^{\prime} O^{C}} C\left(L^{\prime \prime} L^{\prime} ;-1,1\right) \\
& +\left(\delta_{i+} \delta_{j+} Q_{L^{\prime},-2}-\delta_{i-} \delta_{j} P_{L^{\prime}, 2}\right) c\left(L^{\left.\left.\left.\prime \prime \prime L^{\prime} ;-1,-1\right)\right]\right\}}\right. \\
& \underset{\text { even } L}{-\sum}\left\{\Gamma_{L}^{(e)} \Gamma_{L^{\prime \prime}}^{(e)}\left[(2 s+1)(2 L+1)\left(2 L^{\prime \prime}+1\right)\right]^{\frac{1}{2}} W\left(s L L^{\prime \prime} ; s L^{\prime}\right)\right. \\
& \underset{L^{\prime}}{\operatorname{even} L^{\prime \prime}}\left(\frac{1}{8}\right)\left[1+(-1)^{L^{\prime}+1}\right]\left[\left(\delta_{i+} \delta_{j-}-\delta_{i-} \delta_{j+}\right) P_{L^{\prime} 0^{\prime}} C\left(L L^{\prime \prime} L^{\prime} ;-1,1\right)\right.
\end{aligned}
$$




$$
\left.\left.-\left(\delta_{i+} \delta_{j+} P_{L^{\prime},-2}-\delta_{i-} \delta_{j}-P_{L^{\prime}, 2}\right) C\left(L^{\prime \prime} L^{\prime} ;-1,-1\right)\right]\right\} \quad \therefore
$$

Eqs. (4.33) are not the immediate result of taking the trace for $\sigma_{N_{\mu \nu}}$, but follow from the trace after use of the symmetry of the C's and the W's. The sum over $M^{\prime}$ involved in the definition of the density matrix has been carried out and only the polarization tensor components which are given remain. The evaluation of this sum eliminates some of the possible values of $L$ ' which have not been explicitly excluded; however, the C's ensure that these values of $L^{\prime}$ do not contribute: In general, it can also be seen from these equations that the division into symmetric and antisymmetric parts also separates the dependence on the polarization. tensors of different rank as is determined by the factor of $\frac{1}{2}\left[1 \pm(-1)^{L^{\prime}}\right]$. For $L^{\prime}=0$, we note also that these equations are in agreement with Eqs. (4.19).

For an initially unpolarized electron beam the nonzero contributions to $\Sigma$ come from $\sigma_{e_{4}}, \sigma_{e_{i} 4}^{(A)}$, and $\sigma_{e_{i . j}}^{(S)}$, as given by Eqs. (4.18). The contributions to the final polarization of the electron beam come from $\left(Q_{e}^{(S)}\right)_{i 4}$ and $\left(Q_{\mathrm{e}}^{(A)}\right)_{i j}$ as given by Eqs. (4.2.1). An actual evaluation of $\Sigma$ and the final polarization of the electron beam may be carried out explicitly as indicated in Eq. (4.11) and (4.16), respectively, using Eqs. (4.18), (4.21), and (4.33). However, an explicit evaluation is unnecessary in determining experimental conditions for which the time-reversal noninvariant terms might be observed.

Practical considerations limit the rank of the polarization tensors to $L^{\prime}<3$, since the population of a given polarization configuration decreases with increasing rank of the tensor. (These configurations are produced only with large momentum transfer, or emission or absorption of radiation of high multipolarity.) This limitation eliminates the use of the $\Gamma^{(m)} \Gamma^{(e)}$ interference term which occurs in $\sigma_{N i j}^{(S)}$ since this term contributes only for tensors of odd rank $L^{\prime}>2$.

For polarization tensors of rank $L^{\prime}=1$, contributions which depend on $\Gamma^{(e)}$ occur in $\sigma_{N_{i} 4}^{(A)}$ and $\sigma_{N i j}^{(A)}$. The dependence of $\sigma_{N i j}^{(A)}$ on $\Gamma^{(e)}$, however, is also accompanied by the same dependence on $\Gamma^{(m)}$. Thus it does not 
provide a means of observing the $\Gamma^{(e)}$ term. Only $\sigma_{\mathrm{Ni}}^{(\mathrm{A})}$, which depends on the interference of $\Gamma^{(c)}$ and $\Gamma^{(e)}$, provides a means of observing $\Gamma^{(e)}$, since the dependence on $\Gamma^{(m)}$ occurs only for tensors of even rank. Therefore, since the only antisymmetric contribution of the electron beam with the same indices is $\sigma_{e i 4}^{(A)}$, the suggestion is to look for asymmetries in the cross section when the target has a vector polarization in a direction perpendicular to that of the momentum transfer.

For polarization tensors of rank $L^{\prime}=2$, contributions which depend on $\Gamma^{(e)}$ occur in $\sigma_{N_{i 4}}^{(s)}$ and $\sigma_{N_{i j}}^{(s)}$. The dependence of $\sigma_{N_{i j}}^{(s)}$ on $\Gamma^{(e)}$ is accompanied by a dependence on $\Gamma^{(m)}$ for tensors of the same rank. Thus it does not provide a means of observing the $\Gamma^{(e)}$ term. However, $\sigma_{\mathrm{N} i 4}^{(A)}$ which depends on the interference of $\Gamma^{(\mathrm{c})}$ and $\Gamma^{(\mathrm{e})}$, does provide a means of observing $\Gamma^{(e)}$, since the dependence on $\Gamma^{(m)}$ occurs only for tensors of odd rank. Therefore, since $\left(\boldsymbol{Q}_{\mathrm{e}}^{(\mathrm{S})}\right)_{\text {i } 4}$ does give a nonzero contribution to the polarization of the final electron beam, this suggests that the $\Gamma^{(e)}$ terms might be observed by examining the polarization of the final electron when the target is described by a second rank polarization tensor only.

An explicit evaluation of the asymmetry in the cross section leads to

$$
\text { As } \propto \hat{q} \cdot(\underset{\sim}{r} \times \underset{\sim}{\mathbb{P}}) \text {, }
$$

where $\underset{\sim}{\boldsymbol{P}}$ is the polarization vector of the target. This follows from our definition of the z-axis and

$$
\dot{Q}_{1, \pm 1} \propto \mp\left(Q_{x} \pm i Q_{y}\right)
$$

(The proportionality factor depends on the spin of the nucleus from our definition of the $\left.P_{L M^{*}}\right)$ The proportionality factor in Eq. (4.34) may be evaluated from Eqs. (4.18) and (4.33) once the proportionality factor between the spherical and Cartesian components of the vector are known. Thus the only component of the polarization vector which gives a contribution to the asymmetry is the component normal to the scattering plane. We also note that the smallest charge multipole moment which contributes is the quadrupole. This is the test which has previously been noted for the deuteron ${ }^{5-8}$, but as we have shown here, the same test may be made 
with any nuclear target with spin greater than one-half.

An explicit evaluation of the final polarization of the electron beam leads to

$$
\begin{aligned}
& \left(\nabla_{e}^{(f)}\right) \propto\left\{(\gamma-1) q_{\ell}\left(\hat{\sim}^{\prime} \times \hat{p}\right) i_{i}+(\gamma-1)\left(\underline{\sim}^{\prime} \times \hat{p}\right) \ell^{r} i\right.
\end{aligned}
$$

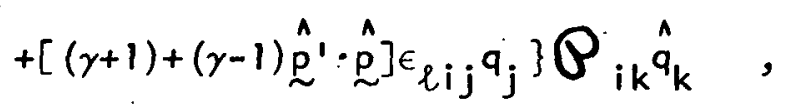

where $\boldsymbol{Q}_{i j}$ is the second rank tensor polarization of the target. The proportionality factor in Eq. (4.35) may be evaluated from Eqs. (4.21) and (4.33) once the proportionality factor between the spherical and Cartesian components of the tensor are known. From Eq. (4.35) it can be seen that the final polarization of the electron beam has components in the scattering plane only if the tensor has a component in the plane containing the normal to the scattering plane and the initial momentum of the nucleus. The component of the polarization of the final electron beam normal to the scattering plane is nonzero only if the tensor has a component in the scattering plane.

It should be pointed out that if the polarization of the target is not described completely,by just a tensor of the rank indicated above, the results of the suggested tests may be ambiguous. Ambiguities may also arise from the higher order processes. However, these ambiguities may be investigated to some extent using the helicity formalism as was pointed out in Chapter 111 . The helicity formalism may be used to investigate the asymmetry in the cross section, since.it is the component of the vector polarization of the target which is normal to the scattering plane that is involved with this asymmetry in the one photon exchange approximation. The transformation of Eq. (3.8) from the c.m. frame to the Breit frame involves a rotation of the initial density matrix (given by a wigner rotation) about the nomal to the scattering plane. Therefore, the component. of the vector polarization along the normal is unaffected by the transformation. Thus it is only necessary to examine any asymmetries in the cross section which may arise from a component of the vector polarization along the normal in the c.m. frame. 
Any ambiguities in the final polarization of the electron beam, however, are not readily investigated with this approach. The final polarization of the electron beam is nonzero only if the target polarization is described by a second rank tensor with components in the scattering plane. Therefore, since the transformation from the c.m. frame to the Breit frame involves a rotation about the normal to the scattering plane through an angle which varies with the energy of the nucleus, it becomes necessary to investigate the contribution of all the components of the tensor polarization to the final polarization of the electron beam. Thus we will confine our attention only to the asymmetry in the cross section.

The calculation of $\Sigma$ from Eq. (3.8) first leads to

$$
\Sigma=\sum_{\substack{\lambda_{e}^{\prime}, \lambda_{N}^{\prime}, \lambda_{e}, \lambda_{N}, \lambda_{N}^{\prime \prime} \\ \lambda_{e}}} f\left(\lambda_{e}^{\prime} \lambda_{N}^{\prime} ; \lambda_{e} \lambda_{N}\right)\left(\frac{1}{2}\right)\left(\rho_{i}^{(0)}\right) f_{N}^{* *}\left(\lambda_{e}^{\prime} \lambda_{N}^{\prime} ; \lambda_{e} \lambda_{N}^{\prime \prime}\right), \lambda_{N}^{\prime \prime},
$$

where the density matrix here is to be taken to have a vector contribution only, so that

$$
\rho_{i}^{(0)}=(2 s+1)^{-1}\left[1+\sum_{M} \cdot \boldsymbol{Q}_{1 M} T_{1 M}^{\dagger}\right] .
$$

In defining the helicity amplitudes, we chose the normal to the scattering plane as the $y$-axis; so, if we take the polarization vector to have a component normal to the scattering plane, the only nonzero part will come from the $y$-component of the cartesian tensor. An explicit evaluation of $T_{\text {IM }}$ from Eq. $(5.10)$ gives

$$
\begin{aligned}
& T_{10}=[s(s+1) / 3]^{-\frac{1}{2}} s_{z}, \\
& T_{1, \pm 1}=\mp[2 s(s+1) / 3]^{-\frac{1}{2}}\left(s_{x} \pm i s_{y}\right),
\end{aligned}
$$

which, together with Eq. $(4.10)$, gives

$$
\begin{aligned}
& \boldsymbol{Q}_{1 U}=[s(s+1) / 3]^{-\frac{1}{2}} Q_{z}, \\
& \boldsymbol{Q}_{1, \pm 1}=\mp[2 s(s+1) / 3]^{-\frac{1}{2}}\left(\boldsymbol{Q}_{x} \pm i \boldsymbol{Q}_{y}\right) .
\end{aligned}
$$


Thus, with the limitation that the polarization vector has a component along the z-axis, Eq. (4.36) becomes

$$
\begin{aligned}
& \Sigma=[2(2 s+1)]^{-1} \underset{\lambda_{e}^{\prime}, \lambda_{e},}{\Sigma}\left|f\left(\lambda_{e}^{\prime} \lambda_{N}^{\prime} ; \lambda_{e} \lambda_{N}\right)\right|^{2} \\
& \lambda_{N}^{\prime}, \lambda_{N} \\
& -i[2(2 s+1) / 3]^{-1}[2 s(s+1)]^{-\frac{1}{2}} \quad y \\
& \left\{\sum _ { \lambda _ { e } ^ { \prime } , \lambda _ { N } ^ { \prime } , \lambda _ { e } } \left[f\left(\lambda_{e}^{i} \lambda_{N}^{\prime} ; \lambda_{e} \lambda_{N}\right) f^{*}\left(\lambda_{e}^{\prime} \lambda_{N}^{\prime} ; \lambda_{e} \lambda_{N}^{\prime \prime}\right) c\left(s i s ; \lambda_{N} 1 \lambda_{N}^{\prime \prime}\right)\right.\right. \\
& \left.\left.\lambda_{N}^{\prime}, \lambda_{N}^{\prime}, \lambda_{e} \lambda_{N}^{\prime \prime}+f\left(\lambda_{e}^{\prime} \lambda_{N}^{\prime} ; \lambda_{e} \lambda_{N}\right) f^{* *}\left(\lambda_{e}^{\prime} \lambda_{N}^{\prime} ; \lambda_{e} \lambda_{N}^{\prime \prime}\right) c\left(s 1 s ; \lambda_{N},-1, \lambda_{N}^{\prime \prime}\right)\right]\right\},
\end{aligned}
$$

which follows after an evaluation of the matrix elements of $T_{1, \pm 1}$. If we interchange the summation variables $\lambda_{N}$ and $\lambda_{N}^{\prime \prime}$ in the last term, make use of the symmetry of the C's, and carry out the sum of $\lambda_{N}^{\prime \prime}$, Eq. $(4.40)$ is

$$
\begin{aligned}
\Sigma= & {[2(2 s+1)]^{-1} \underset{\substack{\lambda_{e}^{\prime}, \lambda_{e} \\
\lambda_{N}^{\prime}, \lambda_{N}}}{\Sigma}\left|f\left(\lambda_{e}^{\prime} \lambda_{N}^{\prime} ; \lambda_{e} \lambda_{N}\right)\right|^{2}+\left[[(2 s+1) / 3]^{-1}[2 s(s+1)]^{-\frac{1}{2}} \boldsymbol{Q}_{y}\right.} \\
& \lambda_{e}^{\prime}, \lambda_{e}, \\
& \lambda_{N}^{\prime}, \lambda_{N}
\end{aligned}
$$

The asymmetry in the cross section, then, is proportional to the imaginary part of the product

$$
f\left(\lambda_{e}^{\prime} \lambda_{N}^{\prime} ; \lambda_{e} \lambda_{N}\right) f^{*}\left(\lambda_{e}^{\prime} \lambda_{N}^{\prime} ; \lambda_{e} \cdot \lambda_{N}+1\right)
$$

The imaginary part of this product is not zero in general, since the symmetry conditions given by Eqs. (3.4), (3.6), and (3.7) do not restrict the f's to be real or to satisfy

$$
f\left(\lambda_{e}^{\prime} \lambda_{N}^{\prime} ; \lambda_{e} \lambda_{N}\right)=f\left(\lambda_{e}^{\prime} \lambda_{N}^{\prime} ; \lambda_{e} \cdot \lambda_{N}+1\right)
$$

Thus, it is possible for the higher order processes to give a nonzero contribution to the asymmetry in the cross section and an observation of a nonzero asymmetry in the cross section would not necessarily indicate a 
violation of time-reversal invariance. This method does not indicate the order of the process which contributes to the asymmetry, however, and it. is possible that the order is large enough that this asymmetry is greatly suppressed. A more detailed investigation of this asymmetry could be made using the one photon exchange as an accurate description of each photon-nucleus vertex for at least the two photon exchange process.

D. Oriented Target and Beam

An explicit calculation of the cross section and the final polarization of the electron beam can be made using Eqs. (4.18), (4.21), (4.22), (4.23), and (4.33). However, these experimental conditions are not likely to be useful for a test of time-reversal noninvariance, since there is no way to easily isolate the contributions of the time-reversal noninvariant terms. From this point of view, the best conditions seem to exist for an initially unpolarized electron beam as discussed in section $C$. 


\section{APPENDIX}

A. Complete Set of Matrices for any Spin-Rotation Group Representations

other methods of constructing a complete set of matrices have been given. (in particular the matrices have often been given as proportional to

$$
S_{L M}(\underset{\sim}{s})=(\underset{\sim}{s} \cdot \underset{\sim}{\nabla})^{L} \cdot r^{L} Y_{L M}(\hat{r})
$$

However, the construction given here seems to be unusual and illustrates the usefulness of starting directly with the transformation properties.

We take the matrices to be spherical tensors $S_{L M}$ which transform according to

where

$$
e^{-i \theta \cdot s} S_{L M} e^{+i \theta \cdot s}=\sum_{M^{\prime}} \boldsymbol{\theta}_{M^{\prime} M}^{L} S_{L M^{\prime}}
$$

$$
\begin{aligned}
& M, M^{\prime}=-L,-L+1, \cdots, L ; \\
& \boldsymbol{\theta}_{M^{\prime} M}^{L}=\left\langle L M^{\prime}\left|e^{-i \theta} \sim \mathcal{\sim}_{L}\right| L M\right\rangle ;
\end{aligned}
$$

and $\underset{\sim}{s}$ is the representation of the angular momentum in a $(2 s+1)$ dimensional space.

This transformation property is not the usual one for spherical tensors but is a direct consequence of restricting the tensors to have matrix elements for the states in the $(2 s+1)$ dimensional spin space only. Also, this restriction leads to a limitation on the maximum rank of the matrices.

Under an infinitesimal transformation we find

$$
\underset{\sim}{\theta} \cdot\left[\underset{\sim}{s}, S_{L M^{\prime}}\right]_{-}=\underset{\sim}{\theta} \cdot \sum_{M^{\prime}} \cdot S_{L M^{\prime}}\left\langle L M^{\prime}|J| L M\right\rangle,
$$

to first order in the $\theta_{1}$. By choosing $\theta$ appropriately, this leads to

$$
\begin{aligned}
& {\left[s_{z}, s_{L M}\right]_{-}=M s_{L M},} \\
& {\left[s_{ \pm}, s_{L M}\right]_{-}=[(L \mp M)(L \pm M+1)]^{\frac{1}{2}} s_{L, M \pm 1},}
\end{aligned}
$$


where $s_{ \pm}=s_{x} \pm$ is $s_{y}$. These commutation relations are sufficient to allow the proof that the matrix elements of the $S_{L M}$ are given by

$$
\left\langle s, \lambda^{\prime}\left|s_{L M}\right| s, \lambda\right\rangle=C\left(s L s ; \lambda M \lambda^{\prime}\right)\left(s\left\|s_{L}\right\| s\right),
$$

where $C\left(s L s ; \lambda M \lambda^{\prime}\right)$ is a Clebsch - Gordon coefficient and $\left(s\left\|S_{L}\right\| s\right.$ ) is a reduced matrix element. This is just the Wigner. - Eckart theorem with the restriction of the states to the spin s states only. From Eq. $(5,3)$ and the properties of the $C^{\prime}$ 's it is easy to show that the $S_{L M}$ are nonzero only if L: $\leq 2 \mathrm{~s}$.

The evaluation of the reduced matix element follows from the definition of the tensors of rank one and the product rule for spherical tensors. We define the first rank tensors by

$$
\begin{aligned}
& s_{10} \equiv s_{z}, \\
& s_{1, \pm 1}=\mp \frac{1}{\sqrt{2}} s_{ \pm},
\end{aligned}
$$

and note that Eq. (5.4b) follows from Eq. (5.4a) and the commutation relations, Eq. (5.2b). The product rule for spherical tensors is

$$
T_{L M^{\prime}}\left(A_{1}, A_{2}\right)=\sum_{M^{\prime}} C\left(L_{1} L_{2} L_{i} ; M^{\prime}, M-M^{\prime}, M\right) T_{L_{1} M^{\prime}}\left(A_{1}\right) \cdot T_{L_{2}, M-M^{\prime}}\left(A_{2}\right) \therefore \cdot
$$

If $T_{L_{1} M^{\prime}}$ and $T_{L_{2} M-M^{\prime}}$ are tensors of the type considered here, it follows that $T_{L M}$ is also. Now for $L=M=L_{1}+L_{2}$ only one $C$ - coefficient is nonzero, i.e. $C\left(L_{1} L_{2} L ; L_{1} L_{2} L\right)=1$. Thus

$$
S_{L L}=\left(-\frac{1}{\sqrt{2}} S_{+}\right)^{L} \text {, }
$$

and the reduced matrix element is found to be

$$
\left(s\left\|s_{L}\right\| s\right)=(2)^{-L / 2}\left(\frac{2 L+1}{2 s+1}\right)^{\frac{1}{2}} \quad\left[\frac{(L !)^{2}(2 s+L+1) !}{(2 s-L) !(2 L+1) !}\right]^{\frac{1}{2}},
$$

where we have used the expression given by Rose ${ }^{26}$ [Eq. (3.19)] for the $c$-coefficients. The other components of the $L$ th rank tensors may be found by using either the commutation rules Eq. (5.2b) with $S_{\text {LL }}$ as given in Eq. (5.5) or from the matrix elements as in Eq. (5.3) with (s\|s $\| s$ ) 
given by Eq. $(5.6)$.

Some simple consequences of this construction are

$$
\begin{aligned}
& S_{L,-M}=(-1)^{M} S_{L M}^{\dagger}, \\
& S_{O O} \equiv 1,
\end{aligned}
$$

and $\quad \operatorname{Tr}\left(S_{L M^{\prime} S^{\prime} M^{\prime}}^{+}\right)=\left(\frac{2 s+1}{2 L+1}\right)\left(s\left\|S_{L}\right\| s\right)^{2} \delta_{L L^{\prime}} \cdot \delta_{M M^{\prime}}$.

Eq. (5.7) follows from Eqs. $(5.2)$ and $(5.4)$ and the reality of the $c$ coefficients; Eq. (5.8) follows from an explicit evaluation of Eq. (5.3) for $L=M=0$; Eq. (5.9) follows from Eqs. (5.3) and (5.7) and the symmetry and orthogonality of the C-coefficients.

From Eqs. (5.7) and (5.9), it is easily shown that the $S_{L M}$ are linearly independent. Thus for each value of $L$ there are $2 L+1$ linearly independent matrices and since $L$ ranges from 0 to $2 L$ there are a total of $(2 s+1)^{2}$ linearly independent matrices. Therefore, the $S_{L M}$ form a complete set for forming any $(2 s+1) \times(2 s+1)$ dimensional matrix.

The matrices which we have used extensively here are simply a renormalization of the $S_{L M}$ given above, defined by

$$
T_{L M}=\frac{(2 L+1)^{\frac{1}{2}}}{\left(s\left\|S_{L}\right\| s\right)} S_{L M} \text {. }
$$

Some of the consequences of the renormalization are

$$
\begin{aligned}
& \left\langle s, \lambda^{\prime}\left|T_{L M}\right| s, \lambda\right\rangle=(-1)^{s-\lambda}(2 s+1)^{\frac{1}{2}} c\left(s s L ; \lambda,-\lambda^{\prime},-M\right), \\
& \operatorname{Tr}\left(T T_{L M} T_{L^{\prime} M^{\prime}}^{+}\right)=(2 s+1) \delta_{L^{\prime}} \delta_{M M^{\prime}} .
\end{aligned}
$$

However, the $T_{L M}$ still satisfy Eqs. (5.7) and (5.8) and form a complete set. The $T_{L M}$ as defined here are in agreement with the Madison Convention 39 .

\section{B. Complete Set of Matrices for any Spin-- Lorentz Group Representations}

The matrices given here are involved in the description of a particle of spin $s$ and rest mass $m$ using wave functions which form a basis for the $(0, s),(s, 0)$, or $(0, s) \oplus(s, 0)$ representations of the Lorentz group. Our primary interests are the expansion of the complete set of matrices in 
terms of spin $s$ angular momentum matrices and their properties under discrete transformations. Consequently, we make no at tempt to dis'cuss the algebra of the matrices. These matrices are a special case of the matrices constructed by Barut, Muzinich, and Williams ${ }^{40}$. Williams ${ }^{15}$ has given a discussion of the algebra of these matrices by expressing them inductively in terms of the spin one-half representation. Alternatively, Weinberg ${ }^{12}$ and $\mathrm{Nelson}$ and $\mathrm{Good}^{16}$ have given methods of construction of the $(s, s)$ representation matrices in terms of the spin s angular momentum matrices; Weinberg's in terms of the expansion of exponential functions of the spin matrices and Nelson and Good's in terms of polynomials derived from the transformation properties of the covariant matrices. The expansion of the complete set of matrices follows in a manner analogous to the expansion of the $(s, s)$ representation given by Nelson and Good and is a consequence of the transformation properties of the matrices and the requirement that they form the basis for an irreducible representation.

The problem of what types of matrices may occur for the irreducible representations is solved by studying the bilinear covariants which may. be built from the wave function basis of the representations. The bilinear covariants are of the form $\varphi^{\dagger} U_{A} X$ where $\varphi$ and $X$ are $(0, s)$ or $(s, 0)$ functions. For the continuous Lorentz group the $(0, s)$ functions transform as

$$
\varphi^{*}=\left(e^{i \tau \cdot \stackrel{s}{\tau}}\right) \varphi,
$$

and the $(s, 0)$ functions as

$$
\varphi^{\prime}=\left(e^{i \tau^{*} \cdot s}\right) \varphi,
$$

where the $T_{i}$ aile tliree cumplex numbers which parametrize the Lorentz transformations. (Since the $\tau_{i}$ are complex, the representations are nonunitary in general.) It should be noted that if $\varphi$ is a $(0, s)$ [or $(s, 0)$ ] function, then $\varphi^{\dagger}$ transforms as $a(s, 0)$ [or $\left.(0, s)\right]$ function. The types of matrices $U_{A}$ are then determined by a clebsch-Gordon analysis of the bilinear covariants:

(i) for $\varphi$ and $x(0, s)$ functions we have $(s, 0) \otimes(0, s)=(s, s)$;

(ii) for $\varphi$ and $X(s, 0)$ functions we have $(0, s) \otimes(s, 0)=(s, s)$;

(IIi) for $\varphi$, an $(s, 0)$ function and for $\chi$, a $(0, s)$ function we have 


$$
(0, s) \otimes(0, s)=\sum_{\substack{k=0 \\ k=0}}^{2 s}(0, k) ;
$$

(iv) for $\varphi$, a $(0, s)$ function and for $x$, an $(s, 0)$ function we have

$$
(s, 0) \otimes(s, 0)=\sum_{k=0}^{2 s}(k, 0),
$$

where $\sum_{k}$ is to be taken to mean a direct sum on all possible values of $k$, an integer between zero and $2 \mathrm{~s}$.

It is possible to make a special choice of the matrices $U_{A}$ so that they are labeled like the components of a Lorentz tensor. Hamermesh 41 has given a discussion of the construction of tensors which form the basis of irreducible representations for various groups. If we write a general transformation of the coordinate four-vector as

$$
x_{\mu}^{\prime}=a_{\mu \nu} x_{\nu},
$$

where $x_{4}$ is $i t$, then $a_{\mu \nu} a_{\mu p}$ is $\delta_{\nu p}$ and $a_{i j}, i a_{i 4}, i a_{4 i}$, and $a_{44}$ are real. Since the reality of the transformation coefficients is unimportant as to what tensor types form bases for irreducible representations of the Lorentz group, we may treat the Lorentz group as the orthogonal group in four dimensions. It should be noted, though, that all irreducible representations of the Lorentz group do not have a corresponding tensor type.

For cases ( $i$ ) and ( $i$ ) above, the tensor is of rank $2 \mathrm{~s}$, symmetric with respect to the interchange of any index pair and with all contractions zero. These are the matrices examined by Nelson and Good ${ }^{16}$ and need no further discussion. Their results, which are of interest here, are

$$
\begin{aligned}
& s_{\mu \nu \rho . . .}=s_{\nu \mu p_{\ldots}}=s_{\mu \rho \nu . . .}=\ldots \text {, } \\
& \mathrm{S}_{\mu \mu \rho}=0 \text {, }
\end{aligned}
$$

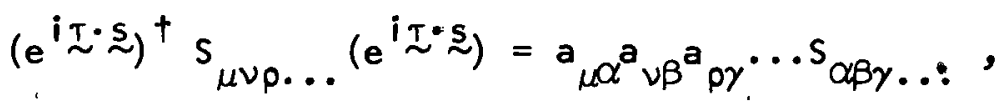

where Eq. (5.16) follows from the transformation properties of the $(0, s)$ functions. (The dagger denotes Hermitian conjugation.). Eqs. (5.14) through (5.16) leave an overall numerical factor unspecified which is chosen so 
that

$$
s_{4 \ldots 4}=(i)^{2 s}
$$

This choice yields the results

$$
\begin{aligned}
& s_{\mu \nu \rho . . .}^{\dagger}= \pm s_{\mu \nu \rho . . .} \text {, } \\
& c_{s} s_{\mu \nu \rho . . . s_{s}^{-1}}=(-1)^{2 s} s_{\mu \nu \rho . . .}^{*} \text {. }
\end{aligned}
$$

The sign in Eq. (5.18) is $+1(-1)$ when there is an even(odd) number of 4 's in the indices; the asterisk denotes complex conjugation. $C_{s}$ is unitary matrix such that

$$
c_{s} s c_{s}^{-1}=-s^{*}
$$

(In the matrix representation where only $s_{2}$ has imaginary elements, $C_{s}$ may also be chosen to have real elements.)

A consequence of Eq. (5.19) is the complement of Eq. (5.16),

$$
\left(e^{i \tau^{*} \cdot s}\right)^{+} s_{\mu \nu \rho . . .}\left(e^{i I^{*} \cdot s}\right)=a_{\mu \alpha^{a} \nu \beta^{a} \rho \gamma} \ldots s_{\alpha \beta \gamma, \ldots}^{\dagger} .
$$

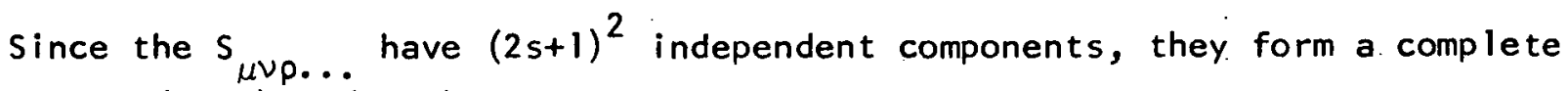
set of $(2 s+1) \times(2 s+1)$ - square matrices.

(The parameter $\mathcal{I}$ may be determined from the transformation coefficients at this point. For spin one-half the matrices $s_{\mu}$ are $S_{4}=i$ and $\Sigma=\ell$, the Pauli spin matrices. The product rule is simple and the exponential may be expanded in a form which is easy to work with. For a space rotation through an angle $\theta$ in the right-hand sense about the direction $\underset{\sim}{\theta} \theta, \underset{\sim}{\tau}$ is $\underset{\sim}{\theta}$ itselr. För a pure Lorentz transformation to a set of axes having velocity $\underset{\sim}{v}$ relative to the initial set of axes, $\mathcal{\sim}$ is $i(v / v)$ arctanh v.)

For cases ( $i i i)$ and ( $i v)$, there is a tensor type for each value of $k$, an integer. By Hamermesh's ${ }^{41}$ prescription, the tensors corresponding to $(0, k)$ and $(k, 0)$ are of rank $2 k$ with symmetry given by the Young pattern which has rows which are $k$ boxes long and columns 2 boxes long. Thus it is a tensor whose indices may be grouped in $k$ pairs, antisymmetric with respect to interchange of indices within a pair and symetric with respect to interchange of one pair with another. 
In addition these tensors must be either self dual or anti-self dual on the antisymmetric indices. This "dualness" is determined by the transformation properties of the tensors. We denote the $(0, k), k \neq 0$, tensor type by

$$
T^{\prime}(k) \mu \nu, \rho \sigma, \ldots,
$$

and the $(k ; 0), k \neq 0$, tensor type by

$$
T^{\prime}(k) \mu \nu, \rho \sigma, \ldots,
$$

where we have grouped the indices in antisymmetric pairs. (We may choose $T_{(0)}=T^{\prime}(0)=1$. In the discussion of these tensors which follows, $k=0$ is excluded.) These tensors transform according to

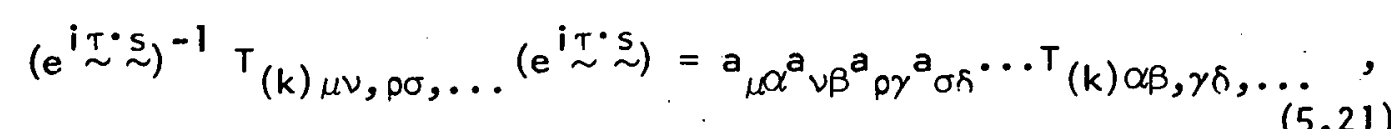

and

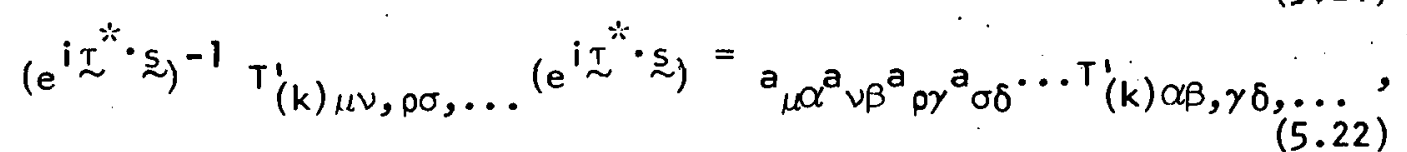

in agreement with the transformation properties of the $(0, s)$ and $(s, 0)$ functions. We note that Eqs. (5.21) and (5.22) are fundamentally different and are not connected in any fashion as are Eqs. (5.16) and (5.20). In order for these tensors to have some sort of dualness and yet be in agreement with Eqs. (5.21) and (5.22) for infinitesimal transformations, it is necessary for

$$
\begin{gathered}
T_{(k) \mu \nu, \rho \sigma, \ldots}=\frac{1}{2} \epsilon_{\mu \nu \omega \tau}{ }^{T}(k) \omega \tau, \rho \sigma, \ldots . \\
=\frac{1}{2} \epsilon_{\rho \sigma \omega \tau}{ }^{T}(k) \mu \nu, \omega \tau, \ldots=\ldots,
\end{gathered}
$$

and

$$
T^{\prime}(k) \mu \nu, p \sigma, \ldots=-\frac{1}{2} \epsilon_{\mu \nu \omega \tau} T^{\prime}(k) \omega \tau, p \sigma, \ldots=\ldots .
$$

Thus we say the $(0, k)$ tensor types are self dual and the $(k, 0)$ tensor types are anti-self dual.

From the symmetry and the dualness of the $(0, k)$ and $(k, 0)$ tensors, we find that the linearly independent components of either tensor type may be written as 


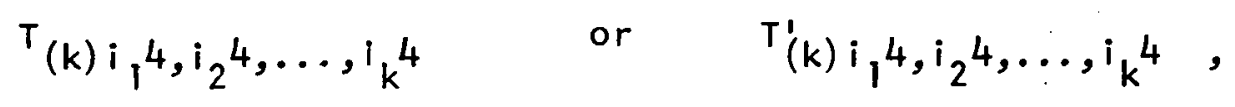

symmetric and contractionless on any pair of space indices. Therefore, except for an overall numerical factor, the independent components form a basis for an irreducible representation of $R_{3}$. We choose the factor so that

$$
\begin{aligned}
& { }^{T}(k) i_{1} 4, i_{2} 4, \ldots, i_{k}{ }^{\prime}=T^{\prime}(k) i_{1} 4, i_{2} 4, \ldots, i_{k}{ }^{4} \\
& =\left\langle s_{i_{1}} s_{i_{2}} \cdots s_{i_{k}}\right\rangle+c_{2}\left\langle\delta_{i_{1} i_{2}} s_{i_{3}} \cdots s_{i_{k}}\right\rangle+\ldots \\
& +c_{k}\left\langle\delta_{i_{1} i_{2}} \cdots \delta_{i_{k-1} i_{k}}\right\rangle \quad \text { (k-even); } \\
& =\left\langle s_{i_{1}} s_{i_{2}} \cdots s_{i_{k}}\right\rangle+c_{1}\left\langle\delta_{i_{1} i_{2}} s_{i_{3}} \cdots s_{i_{k}}\right\rangle+\cdots \\
& +c_{k}\left\langle\delta_{i_{1} i_{2}} \cdots \delta_{i_{k-2} i_{k-1}} s_{i_{k}}\right\rangle \quad \text { (k odd) , }
\end{aligned}
$$

where the c's are to be determined so as to make the tensors contractionless and are therefore real. The angular brackets indicate $1 / k$ ! times a sum over all permutations of the indices. For example,

$$
\begin{aligned}
& \left\langle s_{i} s_{j}\right\rangle=\frac{1}{2}\left(s_{i} s_{j}+s_{j} s_{i}\right), \\
& \left\langle\delta_{i j}\right\rangle=\delta_{i j}, \\
& \left\langle\delta_{i j} s_{k}\right\rangle=1 / 3\left(\delta_{i j} s_{k}+\delta_{i k} s_{j}+\delta_{j k} s_{i}\right) .
\end{aligned}
$$

Since the c's are real and the expressions on the right in Eqs. (5.25) are completely symmetric functions of Hermitian spin matrices, it follows that

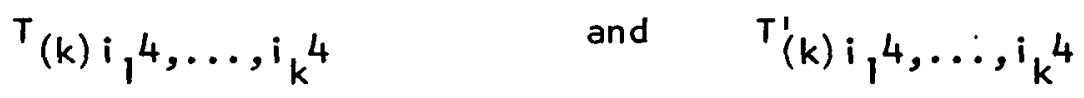

are Hermitian. Thus, using the symmetry of the tensors and Eqs... (5.23) and $(5.24)$, we may show that all the components of $T_{(k)}$ and $T^{\prime}(k)$ are Hermitian. That is, 


$$
\mathrm{T}_{(\mathrm{k}) \mu \nu, \rho \sigma, \ldots}^{\dagger}=\mathrm{T}_{(\mathrm{k}) \mu \nu, \rho \sigma, \ldots,}
$$

and $\quad T^{\prime}(k) \mu \nu, \rho \sigma, \ldots=T^{\prime}(k) \mu \nu, \rho \sigma, \ldots$.

Another. consequence of our choice of factor is

$$
\begin{aligned}
& C_{s}^{\top}(k) \mu \nu, \rho \sigma, \ldots c_{s}^{-1}=(-1)^{k} T^{*}(k) \mu \nu, \rho \sigma, \ldots, \\
& C_{s}{ }^{\prime}(k) \mu \nu, \rho \sigma, \ldots c_{s}^{-1}=(-1)^{k} T^{\prime *}(k) \mu \nu, \rho \sigma, \ldots,
\end{aligned}
$$

which follows directly from the definition of $C_{s}$ and Eqs. (5.25).

Both the $T_{(k)}$ and $T^{\prime}(k)$ have $(2 k+1)$ independent components. Therefore, we have a total of $(2 s+1)^{2}$ linearly independent matrices of either the $(0, k)$ or $(k, 0)$ tensor type, $k=0,1, \ldots, 2 s$. Thus both the $(0, k)$

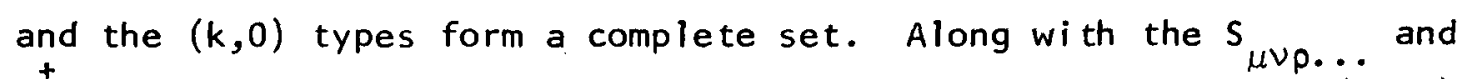
$s_{\mu \nu \rho . . .}^{\dagger}$, these matrices form a complete set for expanding any $(2 s+1)$ square matrix, simultaneously preserving the transformation properties of the matrix.

The matrices that occur with the wave functions in the $(0, s) \oplus(s, 0)$ representation are closely related to the matrices which occur for the irreducible $(0, s)$ and $(s, 0)$ representations. The $(0, s) \oplus(s, 0)$ wave. functions transform as

$$
\begin{aligned}
& \psi^{\prime}=\Lambda \psi, \\
\text { where } & \Lambda=\left(\begin{array}{cc}
e^{i \tau} \underset{\sim}{s} & 0 \\
0 & e^{i \tau^{*} \cdot s}
\end{array}\right),
\end{aligned}
$$

for the continuous Lorentz group. The types of matrices which occur are again given by a clebsch-Gordon analysis of the bilinear covariants:

$$
\begin{aligned}
& {[(0, s) \oplus(s, 0)] \otimes[(0, s) \oplus(s, 0)]} \\
& =2(0,0) \oplus 2(s, s) \oplus \sum_{k=1}^{2 s}[(0, k) \oplus(k, 0)]
\end{aligned}
$$

It should be noted that each of the $(0,0)$ and the $(s, s)$ representations are separately irreducible.

In addition to the covariant matrices it is convenient for some purposes to introduce an auxiliary set of $2(2 s+1)$ - square matrices 


$$
\begin{gathered}
\gamma_{5}=\left(\begin{array}{cc}
-1 & 0 \\
0 & 1
\end{array}\right), \beta=\left(\begin{array}{ll}
0 & 1 \\
1 & 0
\end{array}\right), C=\left(\begin{array}{cc}
0 & c_{s} \\
-c_{s} & 0
\end{array}\right), \\
s=\left(\begin{array}{cc}
s & 0 \\
0 & s
\end{array}\right), \quad \alpha=s^{-1}\left(\begin{array}{cc}
s & 0 \\
0 & -s
\end{array}\right),
\end{gathered}
$$

which satisfy

$$
\begin{aligned}
& C_{\sim}^{\alpha} C^{-1}=\underset{\sim}{\alpha *}, \quad C_{\beta} C^{-1}=-\beta^{*}, \\
& C_{\sim}^{s} C^{-1}=-s_{\sim}^{*}, \quad C_{\gamma_{5}} C^{-1}=-\gamma_{5}^{*} .
\end{aligned}
$$

The bilinear covariants here are of the form $\bar{\psi} \gamma_{A} \bar{\psi}$ where $\bar{\psi}$ is $\psi^{+} \beta$ and the $\gamma_{A}$ are members of the complete set of matrices. For the continuous Lorentz transformations, the bilinear covariants transform as

$$
\bar{\psi}^{\prime} \gamma_{A} \psi^{\prime}=\bar{\psi} \Lambda^{-1} \gamma_{A} \Lambda \psi \text {, }
$$

where we have used $\Lambda^{+} \beta=\beta \Lambda^{-1}$. In order for the $\gamma_{A}$ to be labeled as tensors and yet be in agreement with the transformation rule for the bilinear covariants, the $(s, s)$ matrices must have only off-diagonal blocks while all of the others have only on-diagonal blocks. An appropriate set of matrices is given by

$$
\begin{aligned}
& \gamma_{1}=1, \\
& \gamma_{2}=\gamma_{5}, \\
& \gamma_{3, \mu \nu \rho . . .}=(-i)^{2 s}\left(\begin{array}{ccc}
0 & (-1)^{2 s} s_{\mu \nu \rho .}^{+} \\
s_{\mu \nu \rho .} & 0
\end{array}\right), \\
& \gamma_{4, \mu \nu \rho . .}=-(-i)^{2 s+1}\left(\begin{array}{ccc}
0 & (-1)^{2 s+1} s_{\mu \nu \rho .}^{+} \\
s_{(k+4) \mu \nu, \rho \sigma, \ldots} & & 0
\end{array}\right),
\end{aligned}
$$

$(k=1, \ldots, 2 s)$

where the matrices which make up the smaller blocks are those discussed in connection with the $(0, s)$ and $(s, 0)$ representations. The matrices as 
given here are all Hermitian. Matrices of the form

$$
\gamma_{(k+4) \mu \nu, \rho \sigma, \ldots}^{\prime}=\left(\begin{array}{cc}
T(k) \mu \nu, \rho \sigma, \ldots & 0 \\
0 & -i^{k+1} \cdot T^{\prime}(k) \mu \nu, p \sigma, \ldots
\end{array}\right) \text {. }
$$

need not be included since we may use the dualness of the $T_{(k)}$ 's and $T^{\prime}(k)$ 's to write

$$
\gamma^{\prime}(k+4) \mu \nu, \rho \sigma, \ldots=\frac{1}{2}^{\prime} \epsilon_{\mu \nu \omega \tau} \gamma_{(k+4) \omega \tau, \rho \sigma, \ldots .} .
$$

In addition, the same considerations give the dualing property

$$
\begin{gathered}
\gamma_{(k+4) \mu \nu, p \sigma, \ldots}=\frac{1}{2} \epsilon{ }_{\mu \nu \omega r} \gamma_{5} \gamma_{(k+4) \omega \tau, \rho \sigma, \ldots} \\
=-\frac{1}{2} \epsilon_{\rho \sigma \omega \tau} \gamma_{5} \gamma_{(k+4) \mu \nu, \omega \tau}, \ldots=\ldots .
\end{gathered}
$$

We note also that

$$
\gamma_{4, \mu \nu \rho . . .}=\mathrm{i} \gamma_{5} \gamma_{3, \mu \nu \rho . . .}
$$

The number of linearly independent components of the matrices defined in Eqs. (5.31) is found in a straightforward fashion from the matrices in the sub blocks and Eq. (5.32). The matrices defined by Eqs. (5.31c) and (5.31d) each have $(2 s+1)^{2}$ linearly independent components; the matrices defined by Eq. ( $5.3 l e)$ have $(2 k+1)$ linearly independent components for each value of $k$. Thus, there are a total of $4(2 s+1)^{2} 1$ inearly independent matrices, and the matrices defined by Eqs. (5.31) form a complete set for expanding any $2(2 s+1)$ - square matrix. One further consequence of Eq. $(5.31)$ is

$$
\gamma_{3,44 \ldots 4^{\prime} \cdots \beta} B
$$

(Complete sets of matrices have been developed explicitly for spins onehalf, one ${ }^{42}$, and three-halves ${ }^{36}$. The matrices defined by Eqs. (5.31a) through (5.31d) agree with these special cases, but the matrices given by Fq. (5.31e) in general differ by an overall factor.)

For completeness we give the transformation properties of the bilinear covariants. The $(s, 0) \oplus(0, s)$ wave functions transform simply under space and time reflections as well as the transformations of the continuous 
Lorentz group. For the space reflection

$$
x_{i}=-x_{i}, t^{\prime}=t,
$$

the wave function transformation rule is

$$
\psi^{\prime}\left(x^{\prime}\right)=\eta_{P} \beta \psi(x) \text {. }
$$

For the time reflection

$$
x_{i}^{\prime}=x_{i}, t^{\prime}=-t,
$$

the rule

$$
\psi^{\prime}\left(x^{\prime}\right)=\eta_{T}\left[\begin{array}{ccc}
c & \left.\gamma_{5}^{\beta} \psi(x)\right]^{*}
\end{array}\right.
$$

is used. Here $\eta_{P}$ and $\eta_{T}$ are complex numbers such that $\left|\eta_{P}\right|=\left|\eta_{T}\right|=1$. Thus the transformation rules of the bilinear covariants relative to an. arbitrary Lorentz transformation

$$
x_{\mu}^{\prime}=a_{\mu \nu} x_{\nu}
$$

are

$$
\begin{aligned}
& \bar{\psi}^{\prime} \psi^{\prime}=\bar{\psi} \psi \\
& \bar{\psi} \gamma_{5} \psi^{\prime}=(\operatorname{det} a) \bar{\psi} \gamma_{5} \psi \\
& \Psi^{\prime} \gamma_{3, \mu \nu \rho . . .} \psi^{\prime}=\left(a_{44^{\prime}}\left|a_{44}\right|\right)^{2 s} a_{\mu} \alpha^{a}{ }_{\nu \beta}{ }^{a}{ }_{p \gamma} \ldots \bar{\psi} \gamma_{3, \alpha \beta \gamma} \ldots \psi
\end{aligned}
$$

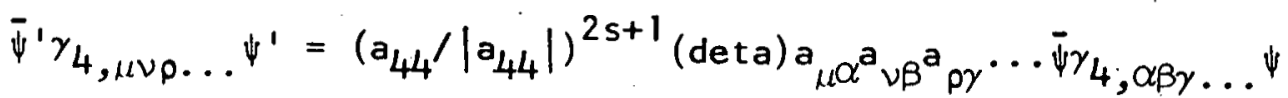

$$
\begin{aligned}
& \bar{\psi}^{\prime} \gamma_{(k+4) \mu \nu, \rho \sigma, \ldots} \psi^{\prime}=\left(a_{44} /\left|a_{44}\right|\right)^{k}{ }_{\mu} \alpha^{a}{ }_{\nu \beta} a_{\rho \gamma} a_{\sigma \delta} \cdots \\
& \bar{\psi} \gamma(k+4) \alpha \beta, \gamma \kappa \ldots \psi
\end{aligned}
$$




\section{Explicit "Boosting" of the Current-- Spin One-half and Spin One}

\section{Spin one-half}

From Table 7 , pages 17 and 18, we find that the possible current terms which are invariant under space reflection are:

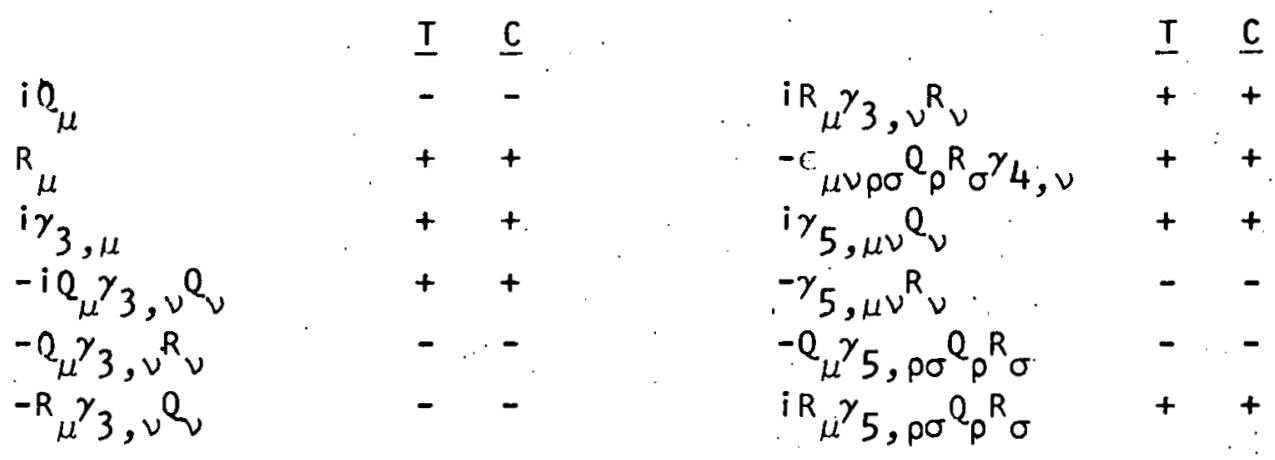

The factors of $\pm i$ and $\pm l$ are in agreement $w i$ th Table 7 . We have repeated the time-reversal and charge conjugation properties of the terms using the convention given for Table 7: A "'+" denotes invariance, a "'-", noninvariance.

The set of matrices given in Appendix B, Eq. (5.31) are given explicitly for spin one-half by

$$
\begin{array}{ll}
\gamma_{1}=\left(\begin{array}{ll}
1 & 0 \\
0 & 1
\end{array}\right), & \gamma_{2}=\left(\begin{array}{cc}
-1 & 0 \\
0 & 1
\end{array}\right), \\
\gamma_{3,4}=\left(\begin{array}{ll}
0 & 1 \\
1 & 0
\end{array}\right), & \gamma_{3, i}=\left(\begin{array}{cc}
0 & i \sigma_{i} \\
-i \sigma_{i} & 0
\end{array}\right), \\
\gamma_{4,4}=\left(\begin{array}{cc}
0 & -i \\
i & 0
\end{array}\right), & \gamma_{4, i}=\left(\begin{array}{cc}
0 & \sigma_{i} \\
\sigma_{i} & 0
\end{array}\right), \\
\gamma_{5, i 4}=\frac{1}{2}\left(\begin{array}{cc}
\sigma_{i} & 0 \\
0 & -\sigma_{i}
\end{array}\right), & \gamma_{5, i j}=\frac{1}{2} \epsilon_{i j k}\left(\begin{array}{cc}
\sigma_{k} & 0 \\
0 & \sigma_{k}
\end{array}\right),
\end{array}
$$

where the $\sigma_{i}$ which appear in the sub-blocks are the $(2 \times 2)$ Pauli matrices. In terms of the auxiliary set of matrices given in Eq. (5.30a), the covariant matrices are

$$
\begin{array}{ll}
\gamma_{3,4}=\beta, & \gamma_{3, i}=-1 \beta u_{i}, \\
\gamma_{4,4}=i \gamma_{5} \beta, & \gamma_{4, i}=2 \beta s_{i}=\beta \sigma_{i},
\end{array}
$$




$$
\gamma_{5, i 4}=\frac{1}{2} \alpha_{i}, \quad \gamma_{5, i j}=\epsilon_{i j k} s_{k}=\frac{1}{2} \epsilon_{i j k} \sigma_{k} .
$$

The connection between the covariant matrices as we have developed them and the standard Dirac matrlces may be found by establishing

$$
\gamma_{3, \mu}=\gamma_{\mu}
$$

and

$$
\gamma_{5, \mu \nu}=\frac{1}{2} \sigma \mu \nu=\frac{1}{4 i}\left[\gamma_{\mu}, \gamma_{\nu}\right]_{-}
$$

and by using Eq. (5.33);

$$
\gamma_{4, \mu}=i \gamma_{5} \gamma_{3, \mu}
$$

The boost operator given in Eq. (2.5) may be written as

$$
B(P)=\exp [s \underset{\sim}{\alpha} \cdot(P / P) \operatorname{arctanh}(P / E)]=[2(\gamma+1)]^{-\frac{1}{2}}[(\gamma+1)+\underset{\sim}{\sim} \cdot P / M],
$$

where $\gamma=E / M$. We will not boost the current terms in general, but will assume that $E^{\prime}=E$. This restriction applies for both the electron and nucleus in both the c.m. and Breit frames. This also restricts $Q_{\mu}$ to $(Q, 0)$. The boosted current terms are therefore given by

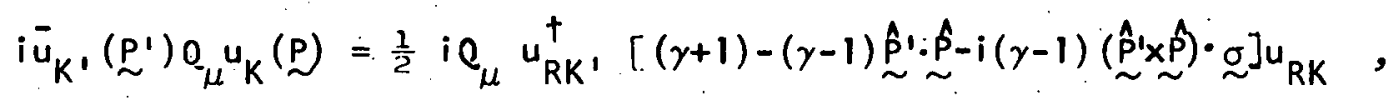

$\bar{u}_{K^{\prime}}\left(\underline{P}^{\prime}\right) R_{\mu^{\prime} U_{K}}(P)=\frac{1}{2} R_{\mu} u_{R^{\prime}}^{\dagger}\left[(\gamma+1)-(\gamma-1) \underset{\sim}{\hat{P}^{\prime}} \cdot \underset{\sim}{\hat{P}}-i(\gamma-1)\left(\underset{\sim}{\hat{P}^{\prime}} \times \underset{\sim}{\hat{P}}\right) \cdot \sigma\right] u_{R K}$,

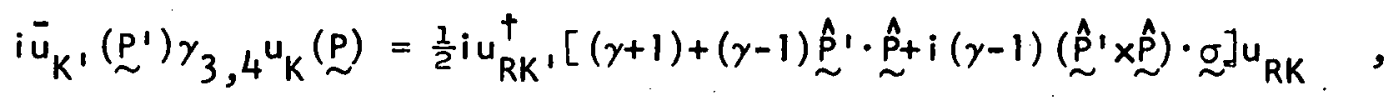

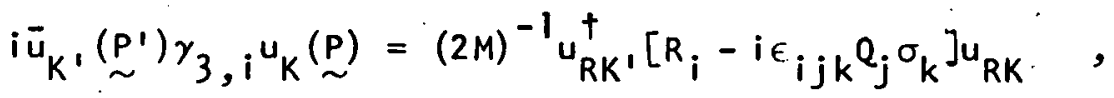

$$
\begin{aligned}
& -i \bar{u}_{K} \cdot\left(P_{\sim}^{\prime}\right) Q_{\mu} \gamma_{3}, \nu^{Q} u_{K}(P)=0 \text {, }
\end{aligned}
$$

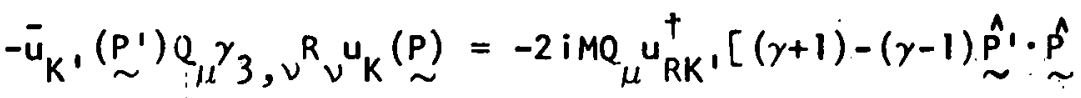

$$
\begin{aligned}
& \left.-i(\gamma-1)\left(\hat{P}^{\prime} \times \hat{\sim}^{\hat{P}}\right) \cdot \sigma\right] u_{\mathrm{RK}},
\end{aligned}
$$




$$
\begin{aligned}
& -\bar{u}_{K^{\prime}}\left(\stackrel{P^{\prime}}{\sim}\right) R_{\mu} \gamma_{3}, \nu^{Q} \nu^{u_{K}} \underset{\sim}{(P)}=0,
\end{aligned}
$$

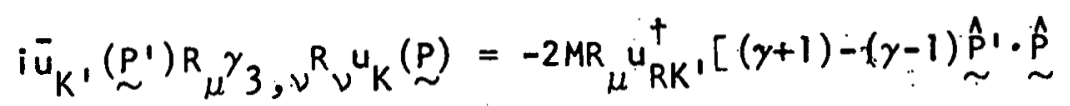

$$
\begin{aligned}
& \left.-i(\gamma-1)\left(\underline{\sim}^{\prime} \times \hat{p}\right) \cdot g\right] u_{R K},
\end{aligned}
$$

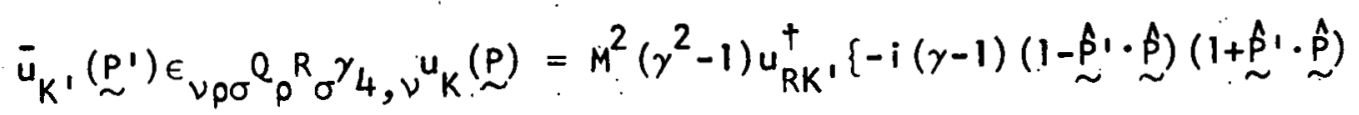

$$
\begin{aligned}
& \left.+\left[(\gamma+1)-(\gamma-1) \underset{\sim}{\hat{\beta}_{1}} \cdot \mathcal{\sim}_{\sim}^{\beta}\right]\left(\underset{\sim}{\hat{\beta}^{\prime}} \times \underset{\sim}{\beta}\right) \cdot \sigma\right] u_{\mathrm{RK}},
\end{aligned}
$$

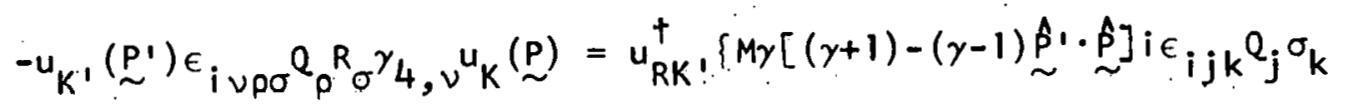

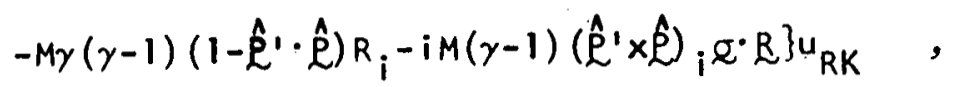

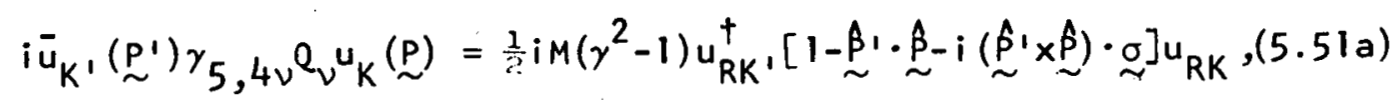

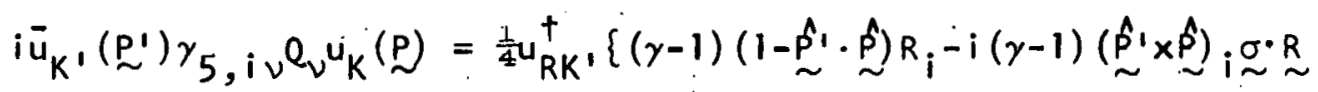

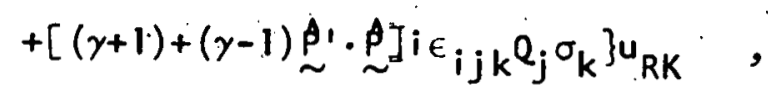

$$
\begin{aligned}
& -\bar{u}_{K} \cdot\left(\stackrel{P}{\prime}^{\prime}\right) \gamma_{5,4 \nu^{R}} \nu^{u_{K}} \underset{\sim}{(P)}=0
\end{aligned}
$$

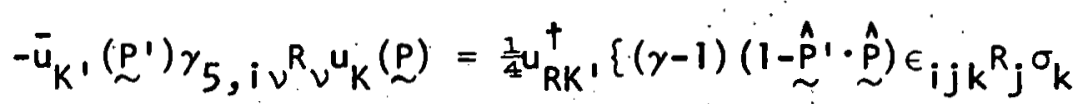

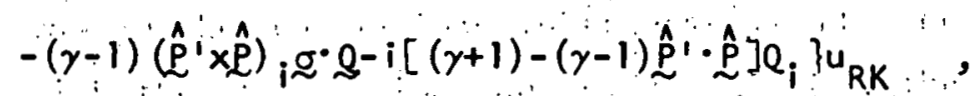

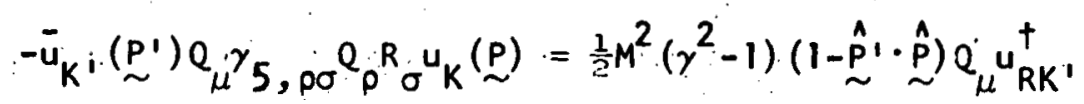

$$
\begin{aligned}
& \left\{-\mathbf{i}\left[(\gamma+1)-(\dot{\gamma}-1) \underline{\beta_{1}} \cdot \underline{\beta}\right]+(\gamma-1)\left(\underline{\alpha}^{\prime} \times \hat{\beta}\right) \cdot g\right\} u_{R K},
\end{aligned}
$$

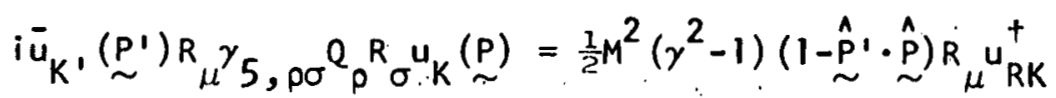

$$
\begin{aligned}
& \left\{-\left[(\gamma+1)-(\gamma-i) \hat{\sim}^{\prime} \cdot{\underset{\sim}{P}}^{\hat{P}}\right]-i(\gamma-1)\left(\underset{\sim}{\hat{P}^{\prime}} \times \underset{\sim}{\hat{P}}\right) \cdot \sigma\right] u_{R K} .
\end{aligned}
$$

Eqs. (5.43) through (5.54) are found by a tedious, but otherwise simple, algebraic procedure. The conservation properties of the various current terms follow easily $\tilde{Q}_{\mu}^{R} R_{\mu}=\underset{\sim}{Q} \cdot \underset{\sim}{R}=0$, and $\underset{\sim}{Q} \cdot\left(\underset{\sim}{P^{\prime}}{ }_{\sim}^{P}\right)=0$. We find that only those terms which are invariant under $P, T$, and $C$, separately, are conserved. Al though these results are also easily found covariantly, this 
does support the validity of boosting the current terms.

The linear relations between the terms is much more easily examined in the Breit frame, where $\stackrel{\sim}{\prime}^{\prime}=-\underset{\sim}{P}=\frac{I}{2} S$. In this frame, the conserved, nonzero terms may be written as

$$
\begin{aligned}
& \bar{u}_{K^{\prime}}\left(P^{\prime}\right) R_{\mu} u_{K}(\mathcal{D})=\gamma R_{\mu} u_{R K^{\prime}}^{\dagger} u_{R K}, \\
& i \bar{u}_{K},\left(P^{\prime}\right) \gamma_{3}, 4^{u_{K}}(P)=i u_{R K}^{\dagger} u_{R K}, \\
& i \bar{u}_{K^{\prime}}\left(P^{\prime}\right) \gamma_{3, i} u_{K} \stackrel{(P)}{\sim}={ }^{\prime \prime}(2 M)^{-i} u_{R K^{\prime}}^{\dagger}\left(-i \epsilon_{i j k} Q_{j} \sigma_{k}\right) u_{R K}, \\
& i \bar{u}_{K^{\prime}}\left(\stackrel{\sim}{\prime}^{\prime}\right) R_{\mu^{\prime}} \gamma_{3, \nu} v_{\nu} u_{K}(P)=-2 \gamma M R u_{\mu} u_{R K}^{\dagger}{ }^{\prime} u_{R K},
\end{aligned}
$$

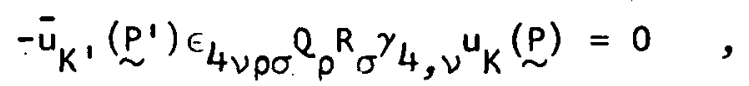

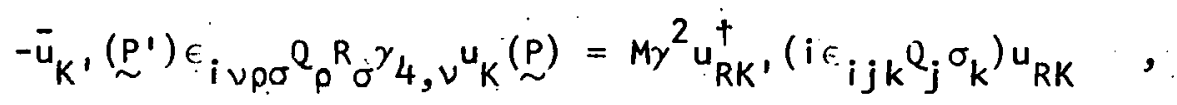

$$
\begin{aligned}
& i \bar{u}_{K} \cdot\left(P^{\prime}\right) \gamma_{5,4 v} \mathcal{Q}_{\nu} u_{K}(P)=i M\left(\gamma^{2}-1\right) u_{R K}^{\dagger}, u_{R K}, \\
& \left.i \bar{u}_{K}{ }^{\prime}\left(\stackrel{P}{ }^{\prime}\right) \gamma_{5, i v} \mathcal{Q}_{\nu_{K}} \underset{\sim}{\sim}\right)=\frac{1}{2} u_{R K^{\prime}}^{\dagger}\left(i \epsilon_{i j k} Q_{j} \sigma_{k}\right) u_{R K},
\end{aligned}
$$

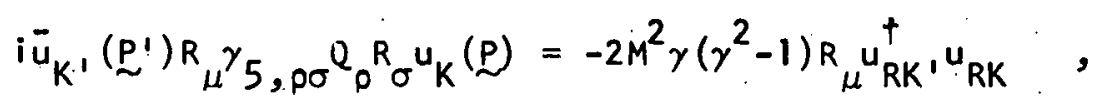

where we have numbered these equations in agreement with the more general boosted terms. With the observation that $R_{\mu}=(0,2 i \gamma M)$, the 1 inear relations between the terms are determined easily from this set of equations. Once these linear relations have been determined in the Breit frame and written in a covariant fashion, these linear relations must hold in all frames of reference.

The components of the boosted current for a spin one-half nucleus as given in Eqs. (2.59) and (2.60), are

$$
j_{4}=i \Gamma_{0}^{(c)}\left(Q^{2}\right)
$$

and

$$
\dot{L}=i \frac{1}{\sqrt{2}} \Gamma_{1}^{(m)}\left(Q^{2}\right) \hat{2} \times \sigma
$$

We note from Eq. (5.44') that the covariant form factor for the term $R_{\mu}$ may be directly related to the physical form factor $\Gamma_{0}^{(c)}$, since only 
the fourth component of $\bar{u}_{\mu} u$ is nonzero. Similarly, from Eqs.. (5.50') we note that the covariant form factor for the term $\epsilon_{\mu \nu \rho \sigma} Q_{\rho}{ }^{R} \sigma_{4, \nu}$ may, be directly related to the physical form factor $\Gamma_{1}^{(m)}$, since only the threevector part of $-\bar{u} \epsilon_{\mu \nu \rho \sigma}{ }^{0} \rho^{R} \sigma^{\gamma}{ }_{4, \nu} u$ is nonzero. The physical significance of the form factors of these terms has previously been pointed out $23,24,43,44$.

2. Spin one

From Table 7, we find that the current terms for spin one that are invariant under space reflections are:

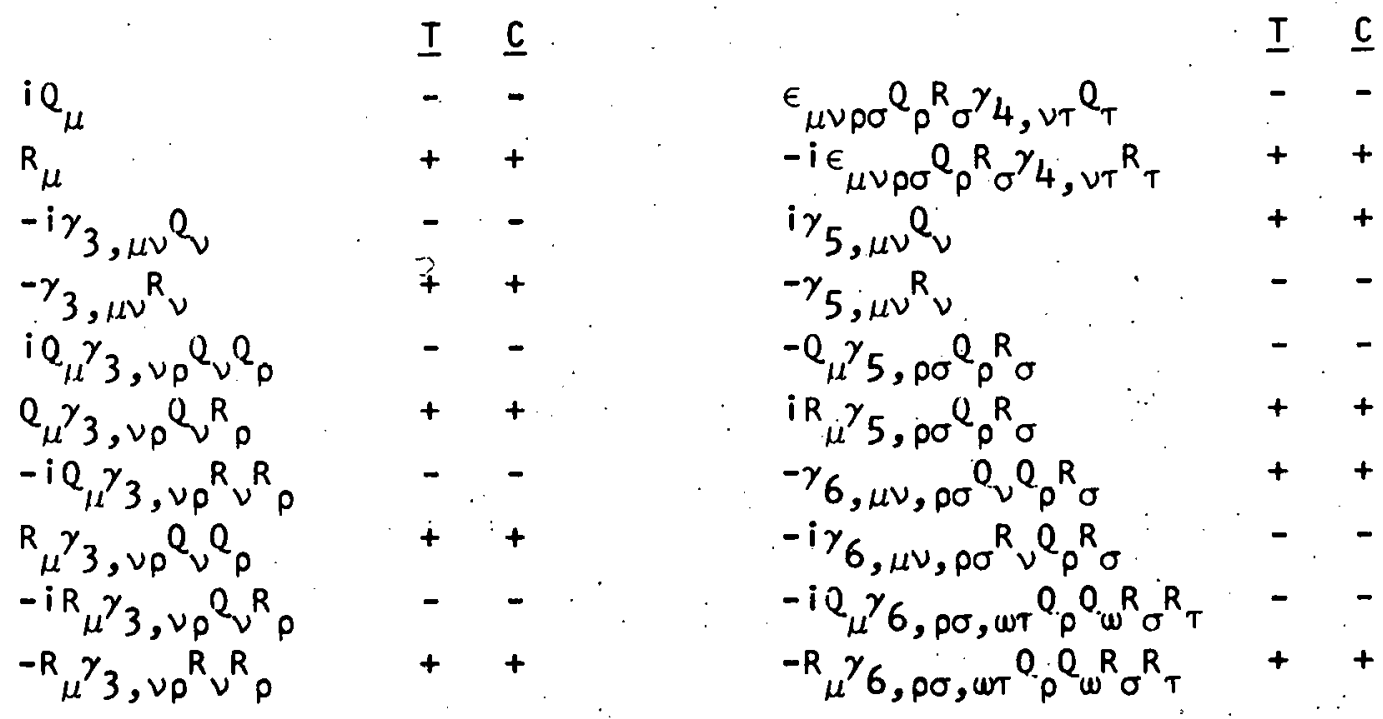

The factors of $\pm i$ and $\pm l$ are in agreement with Table 7. The time-reversal and charge conjugation properties are repeated here for convenience. The covariant spinors satisfy Eq. (2.44), which for spin one particles is given by

$$
\gamma_{3, \mu \nu} P_{\mu} P_{\nu} u_{K}(P)=-M^{2} u_{K}(P)
$$

Use of this equation, $R_{\mu} Q_{\mu}=0$, and the symmetry of matrix indices allows us to show readily that the nonzero terms which give a conserved current are

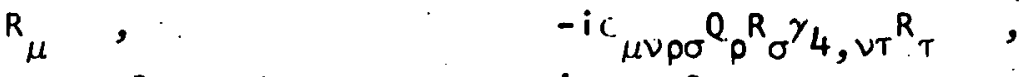

$$
\begin{aligned}
& -\gamma_{3, \mu \nu} \nu^{R}, \quad i \gamma_{5, \mu \nu} v^{2} \text {, }
\end{aligned}
$$

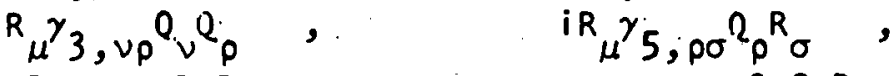

$$
\begin{aligned}
& -R_{\mu} \gamma_{3, \nu \rho}{ }^{R} \nu_{\rho} \quad, \quad \ldots \quad-\gamma_{6}, \mu \nu, \rho \sigma^{Q} Q_{\rho} R_{\sigma} ., \\
& \epsilon_{\mu \nu \rho \sigma} Q_{\rho}{ }^{R} \sigma^{\gamma}{ }_{4, \nu \tau} Q_{T} \quad, \quad, \quad-R_{\mu} \gamma_{6, \rho \sigma, \omega \tau} Q_{\rho} Q_{\omega}{ }^{R} \dot{R}_{T}
\end{aligned}
$$


The terms which are still questionable are

$$
-\gamma_{5, \mu \nu^{R} \nu}, \quad-i \gamma_{6}, \mu \nu, \rho \sigma^{R} \nu_{\rho}^{Q_{\sigma}} \cdot
$$

Use of the equation which the spinors satisfy allows us to show

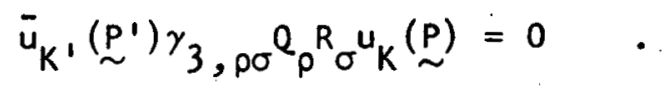

We will boost these terms in the Breit frame only and will use the boosted terms to determine the linear relations between the terms and the current conservation properties of the last two terms.

The set of matrices for spin one given in Appendix B, Eqs. (5.31), are explicitly

$$
\begin{aligned}
& \gamma_{1}=\left(\begin{array}{ll}
1 & 0 \\
0 & 1
\end{array}\right), \quad \gamma_{2}=\left(\begin{array}{rr}
-1 & 0 \\
0 & 1
\end{array}\right), \\
& \gamma_{3,44}=\left(\begin{array}{ll}
0 & 1 \\
1 & 0
\end{array}\right), \quad \gamma_{3, i 4}=\gamma_{3,4 i}=\left(\begin{array}{cr}
0 & i s_{i} \\
-i s_{i} & 0
\end{array}\right) \text {, } \\
& \gamma_{3, i j}=\left(\begin{array}{cc}
0 & \delta_{i j}-s_{i} s_{j}-s_{j} s_{i} \\
\delta_{i j}-s_{i} s_{j}-s_{j} s_{i} & 0
\end{array}\right) \text {; } \\
& \gamma_{5, i 4}=\left(\begin{array}{cc}
s_{i} & 0 \\
0 & -s_{i}
\end{array}\right), \gamma_{6, i 4, j 4}=\frac{1}{2}\left(\begin{array}{cc}
s_{i} s_{j}+s_{j} s_{i}-\frac{4}{3} \delta_{i j} & 0 \\
0 & s_{, j} s_{j}+s_{j} s_{i}-\frac{4}{3} \delta_{i j}
\end{array}\right) \text {. }
\end{aligned}
$$

The matrices $\dot{\gamma}_{4, \mu \nu}$ and the other components of $\gamma_{5, \mu \nu}$ and $\gamma_{6, \mu \nu, p \sigma}$ are related to these matrices by

$$
\begin{aligned}
& \gamma_{4, \mu \nu}=i \gamma_{5} \gamma_{3, \mu \nu}, \\
& \gamma_{5, i j}=-\epsilon_{i j k} \gamma_{5} \gamma_{5, k 4}, \\
& \gamma_{6, i j, k 4}=-\epsilon_{i j l} \ell_{5} \gamma_{6, \ell 4, k 4},
\end{aligned}
$$

and

$$
\gamma_{6, i j, k \ell}=c_{i j m} c_{k \ell, n^{\gamma}, m^{4}, n^{4}} \text {. }
$$

In terms of the auxiliary set of matrices given in Eq. (5.3.0), the covariant matrices are 


$$
\begin{aligned}
& \gamma_{1}=1, \gamma_{2}=\gamma_{5}, \\
& \gamma_{3,44}=\beta, \gamma_{3, i 4}=\gamma_{3,4 i}=-i \beta \alpha_{i}, \gamma_{3, i j}=-\beta\left(s_{i j}+\frac{1}{3} \delta_{i j}\right), \\
& \gamma_{4,44}=i \gamma_{5} \beta, \gamma_{4, i 4}=\gamma_{4,4 i}=\beta s_{i}, \gamma_{4, i j}=-i \gamma_{5} \beta\left(s_{i j}+\frac{1}{3} \delta_{i j}\right) \\
& \gamma_{5, i 4}=\alpha_{i}, \gamma_{5, i j}=\epsilon_{i j k} s_{k}, \\
& \gamma_{6, i 4, j 4}=\frac{1}{2} s i j, \gamma_{6, i j, k 4}=-\frac{1}{2} \epsilon_{i j l} \gamma_{5} s_{k l}, \gamma_{6, i j, k l}=\frac{1}{2} \epsilon_{i j m} \epsilon_{k l n} s_{m n},
\end{aligned}
$$

where we have introduced the special notation

$$
s_{i j}=s_{i} s_{j}+s_{j} s_{i}-\frac{4}{3} \delta_{i j}
$$

These matrices may be related to the set introduced by Sankaranarayanan and Good ${ }^{42}$, which we denote as $\gamma_{A}^{(S)}$,

$$
\begin{aligned}
& \gamma_{3, \mu \nu}=\gamma_{3, \mu \nu}^{(s),} \\
& \gamma_{5, \mu \nu}=-\frac{1}{6} \gamma_{5, \mu \nu}^{(s),} \\
& \gamma_{6, \mu \nu, \rho \sigma}=-\frac{1}{6} \gamma_{6, \mu \nu, \rho \sigma},
\end{aligned}
$$

where $\quad \gamma_{5, \mu \nu}^{(s)}=i\left[\gamma_{3, \mu \lambda}, \gamma_{3 ; \nu \lambda}\right]_{-}$.

and

$$
\gamma_{6, \mu \nu, \rho \sigma}^{(s)}=\left[\gamma_{3, \mu \rho}, \gamma_{3, \nu \sigma}\right]_{+}+2 \delta_{\mu \rho} \delta_{\nu \sigma}-\left[\gamma_{3, \nu \rho}, \gamma_{3, \mu \sigma_{+}}\right]_{-2 \rho_{\nu \rho} \delta_{\mu \sigma}}
$$

The boost operator for spin one may be written in the polynomial form

$$
\begin{aligned}
B(P) & \left.\left.=(\gamma+1)^{-1}[(\gamma+1)+(\gamma+1) \underset{\sim}{\alpha} \cdot \underset{\sim}{P} / M)+\underset{\sim}{\alpha} \underset{\sim}{P / M}\right)^{2}\right] \\
& =(\gamma+1)^{-1}\left\{\left[\frac{1}{3}(\gamma+1)(2 \gamma+1)+\frac{1}{2} s_{i j}\left(P_{i} / M\right)\left(P_{j} / M\right)\right]+(\gamma+1) \underset{\sim}{\alpha} \cdot(P / M)\right\},
\end{aligned}
$$

where $\gamma=E / M$.

In the Breit frame, the boosted form of the terms which have undetermined conservation properties are

$$
-\bar{u}_{K},\left(P^{\prime}\right) \gamma_{5,4 \nu^{R} u_{K}}(\dot{P})=0
$$


73

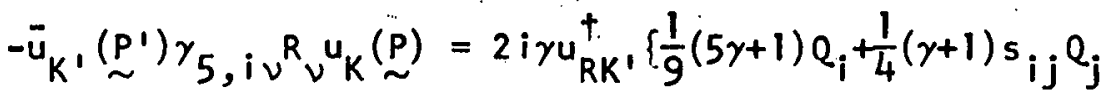

$$
\begin{aligned}
& \left.+\left[16 M^{2}(\gamma+1)\right]^{-1} Q_{i} s k Q_{j} Q_{k}\right\} u_{R K}
\end{aligned}
$$

and

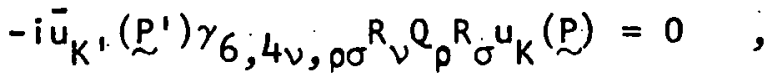

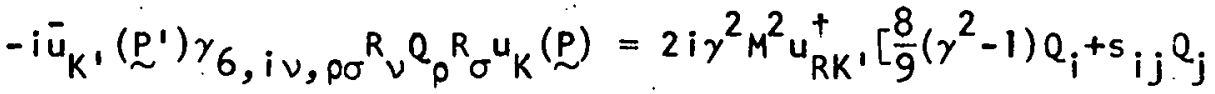

$$
\begin{aligned}
& \left.+\left(4 M^{2}\right)^{-1} Q_{i} Q_{j} Q_{k} s_{j k}\right] u_{R K}
\end{aligned}
$$

From these expressions, it can easily be seen that neither term is conserved. We will confine our attention to those terms which do give a conserved current and will not examine these two terms further.

The boosted form of the conserved terms are

$$
\begin{aligned}
& \bar{u}_{K^{\prime}}\left(P_{\sim}^{\prime}\right) R_{4^{\prime}} u_{K}(P)=2 i \gamma \mathrm{Mu}_{R K^{\prime}}^{\dagger}\left[\frac{1}{3}\left(4 \gamma^{2}-1\right)+\left(4 M^{2}\right)^{-1} Q_{i} Q_{j} s_{i j}\right] u_{R K},(5.61 a) \\
& \bar{u}_{K} \cdot \stackrel{\left(P^{\prime}\right)}{\sim} R_{i} u_{K}(P)=0, \\
& -\bar{u}_{K},\left(P^{\prime}\right) \gamma_{3}, 4 \nu^{R} \nu^{u_{K}}(P)=-2 i \gamma M u_{R K}^{\dagger} \cdot u_{R K}
\end{aligned}
$$

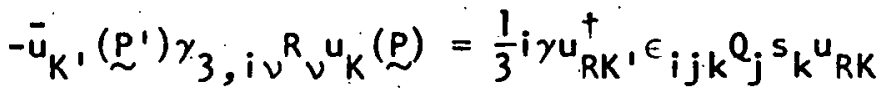

$$
\begin{aligned}
& \bar{u}_{K}\left(P^{\prime}\right) R_{4} \gamma_{3, \nu \rho} Q_{\nu} Q_{\rho} u_{K}(D)=-2 i \gamma M u_{R K}^{+} \cdot\left[\frac{1}{3}\left(4 M^{2}\right)\left(\gamma^{2}-1\right)+Q_{i} Q_{j} s_{i j}\right] u_{R K}
\end{aligned}
$$

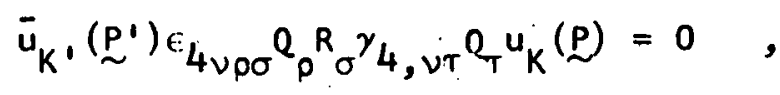

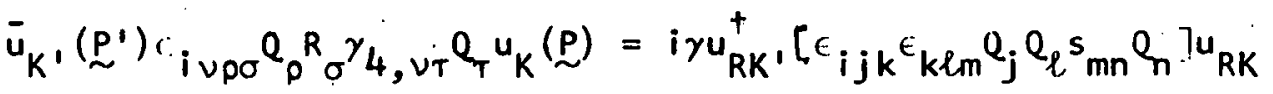

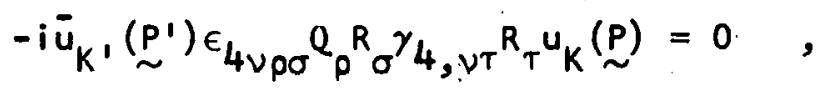




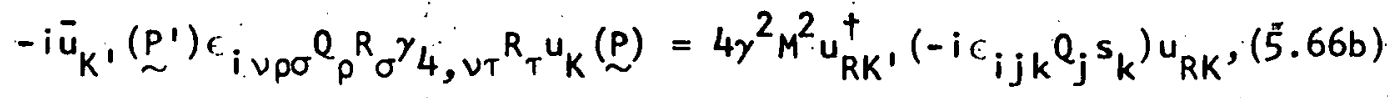

$$
\begin{aligned}
& \left.i \bar{u}_{K^{\prime}}\left(P_{\sim}^{\prime}\right) \gamma_{5,4 \nu^{2}} u_{\nu_{K}} \stackrel{P}{\sim}\right)^{\prime}=i u_{R K^{\prime}}^{\dagger}\left[(4 M 9)(5 \gamma+1)\left(\dot{\gamma}^{2}-1\right)\right. \\
& \left.+(2 M)^{-1} \gamma Q_{i} Q_{j} s_{i j}\right] u_{R K},
\end{aligned}
$$

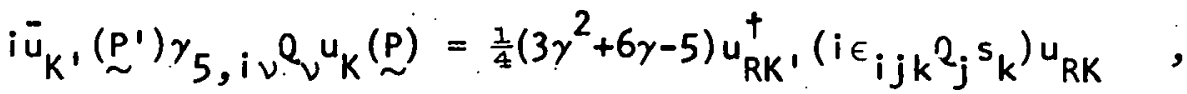

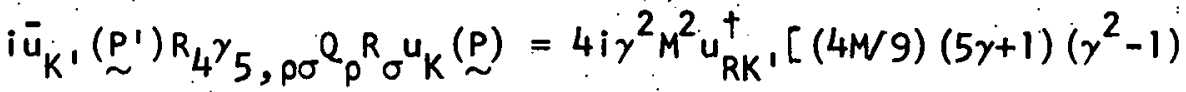

$$
\begin{aligned}
& \left.+(2 m)^{-1} \gamma Q_{i} Q_{j} s_{i j}\right] u_{R K} \text {, }
\end{aligned}
$$

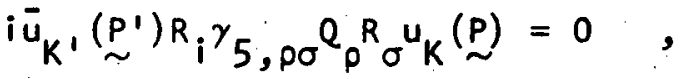

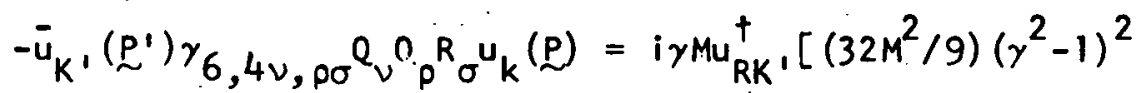

$$
\begin{aligned}
& \left.+\frac{1}{3}\left(2 \gamma^{2}-1\right) Q_{i} Q_{j} s_{i j}\right] u_{R K} \text {, }
\end{aligned}
$$

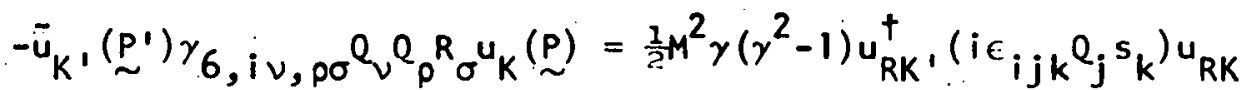

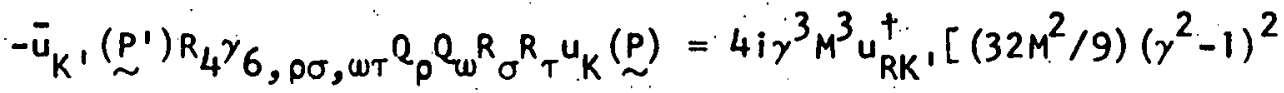

$$
\begin{aligned}
& \left.+\frac{1}{3}\left(2 \gamma^{2}-1\right) Q_{i} Q_{j} s_{i j}\right] u_{R K} \text {, }
\end{aligned}
$$

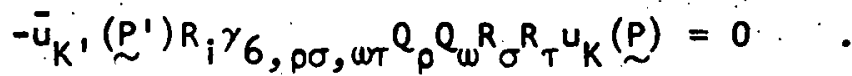

The components of the boosted current for a spin one nucleus as given in Eqs. (2.59) and (2.60), are

$$
j_{4}=i \Gamma_{0}^{(c)}+(3 \sqrt{2} / 4) i \Gamma_{2}^{(c)} Q_{i} \hat{Q}_{j}{ }_{i j}
$$

and $\quad j_{i}=(\sqrt{3} / 2) i \Gamma_{1}^{(m)}(\underbrace{\hat{Q} x \dot{s})}_{\sim}{ }_{i}+(\sqrt{3} / 2) i \Gamma_{2}^{(e)}\left(\epsilon_{i j k} \epsilon_{k \ell m} \hat{Q}_{j} \hat{Q}_{\ell} s_{m n} Q_{n}\right)$.

Comparison with Eqs: (5.61) through (5.70) shows that only the charge quadrupole term, $\hat{Q}_{i} \hat{Q}_{j} s_{i j}$, does not have a covariant analog: The covariant analog of the charge monopole is the term ${ }_{\mu}{ }_{\mu}{ }_{3}, p \sigma^{R}{ }_{\rho}{ }^{R}$; of the magnetic

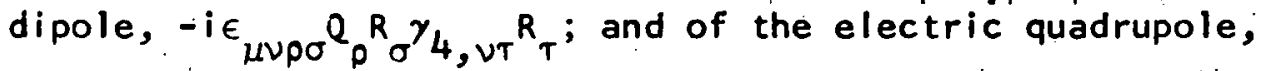

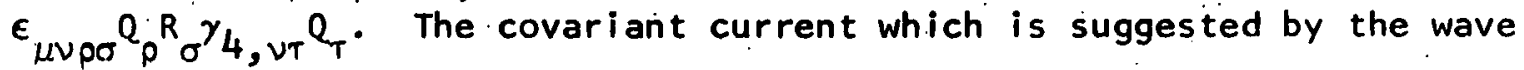
equation for a spin one particle in an external electromagnetic field 
given by Shay and $\operatorname{cood}^{45}$ is

$$
J_{\mu}=\Lambda_{1}\left(\gamma_{3}, \mu \nu \nu_{\nu}+R_{\mu}\right)+i \Lambda_{2} \gamma_{5}, \mu \nu v_{\nu}+\Lambda_{3} \gamma_{6}, \mu \nu, \rho \sigma \nu^{Q}{ }^{R_{0}}{ } \sigma
$$

as was pointed out by Eeg ${ }^{18}$. An examination of Eqs. (5.61), (5.62), (5.67) and (5.69) reveals that the terms multiplied by the form factors are linearly independent and that the set of form factors is nonredundant. 
VI. REFERENCES

1. J. Bernstein, G. Feinberg, and T. D. Lee, Phys, Rev. 139, B1650 (1965).

2. N. Christ and T. D. Lee, Phys. Rev. 143, 1310 (1966).

3. S. Rock, Search for T-Violation in the Inelastic Scattering of Electrons from Polarized Proton Target, (thesis), University of California, Berkeley (1970).

4. V. Glaser and B. Jaksic, Nuovo Cimento 5, 1197 (1957).

5. D. Schildknecht, Z. Physik 185, 382 (1965); ibid. 201, 99 (1967).

6. R. K. Umerjee, Nuovo Cimento 54A, 155 (1968).

7. V. M. Dubovik and A. A. Cheshkov, Soviet Phys.--JETP 24, 111 (1967);

V. M. Dubovik, E. P. Likhtman, and A. A. Cheshkov, Soviet Phys.--JETP 25, $464(1967)$.

8. K. Y. Lin, Nucl. Phys. B18, 162 (1970).

9. R. Prepost, R. M. Simonds, and B. H. Wiik, Phys. Rev. Letters 21, $1271(1968)$.

10. G. Leibbrandt, Nuovo Cimento 59, 153 (1970).

11. H. Joos, Fortschr. Physik 10, 65 (1962).

12. S. Weinberg, Phys. Rev. 133, B1318 (1964):

13. D. L. Weaver, C. L. Hammer, and R. H. Good, Jr., Phys: Rev. 135, B241 (1964).

14. T. J. Nelson and R. H. Good, Jr., Rev. Mod. Phys. 40, 508 (1968).

15. D. N. Williams, in Lectures in Thenretical Physics (summer, 1964), edited by W. E. Brittin and A. O. Barut (University of Colorado Press, Boulder, 1965).

16. T. J. Nelson and R. H. Good, Jr., J. Math Phys. 11, 1355 (1970).

17. W. R. Theis, Nuovo Cimento 45A, 124 (1966).

18. J. 0. Eeg, Preprint [abotract published in Physica Norvegica $\underline{4}$, $252(1970)]$.

19. M. Gourdin, Nuovo Cimento 36, 129 (1965).

20. M. Gourdin and J. Micheli, Nuovo Cimento 40A, 225 (1965). 
21. T. A. Weber and S. A. Williams, J: Math Phys. 6, 1980 (1965).

22. D. R. Yennie, M. M. Lévy, and D. G. Ravenhall, Rev. Mod. Phys. 29, 144 (1957).

23. F. J. Ernst, R. G. Sachs, and K. C. Wali, Phys. Rev. 119, 1105 (1960).

24. R. G. Sächs, Phys. Rev, 126, 2256 (1962).

25. L. Durand, 111 , P. C. DeCelles, and R. B. Marr, Phys. Rev. 126, 1882 (1962).

26. M. E. Rose, Elementary Theory of Angular Momentum (John Wiley and Sons, Inc., New York, 1957).

27. J. H. Scofield, Phys. Rev. 139, 847 (1965).

28. M. E. Rose, Multipole Fields (John Wiley and Sons, Inc., New York, 1955).

29. J. D. Jackson, Classical Electrodynamics (John Wiley and Sons, Inc., New York, 1962), Chapter 16.

30. J. M. Blatt and V. F. Weisskopf, Theoretical Nuclear Physics (John Wiley and Sons, Inc.., New York, 1952).

31. R. H. Pratt, J. D. Walecka, and T. A. Griffy, Nucl. Phys. 64, 677 (1965).

32. H. Überall and P. Uginčius, Phys. Rev. 178, 1565 (1969).

33. L. J. Weigert and M. E. Roşe, Nucl. Phys. 51, 529 (1964).

34. H. Überall, Electron Scattering from Complex Nuclei, Part A (Academic Press, New York, 1971).

35. M. Jacob and G. C. Wick, Ann. Phys. (N.Y.) I, 404 (1959).

36. D. Shay, H. S. Song, and R. H. Good, Jr., Nuovo Cimento Suppl. 3, 455 (1965).

37. U. Fano, Statistical Matrix Techniques and Their Application to the Directional Correlations of Radiations, National Bureau of Standards Report No. 1214 (1951)[unpublished].

38. L. C. Biedenharn, Ann. Phyș. (N.Y.) 4, 104 (1958); ibid. 6, 399 (1959).

39. H. H. Barschall and W. Haeberli, editors, Polarization Phenomena in Nuclear Reactions (University of Wisconsin Press, Madison, 1971). 
78

40. A. 0. Bart, I. J. Muzinich, and D. N. Williams, Phys. Rev. 130, 442 (1963).

41. M. Hamermesh, Group Theory (Addison-Wesley Publishing Co., Inc., Reading, 1962).

42. A. Sankaranarayanan and R. H. Good, Jr., Nuovo Cimento 35, 1303 (1965).

43. K. J. Barnes, Phys. Letters 1, 166 (1962).

44. L. N. Hand, D. G. Miller, and R. Wilson, Rev. Mod. Phys. 35, 335 (1963.).

45. D. Shay and R. H. Good, Jr., Phys. Rev. 179, 1410 (1969). 
VII. ACKNOWLEDGMENTS

The author wishes to thank Professor R. H. Good, Jr., for his help and advice on this problem and other research. The author also. wishes to express his gratitude to his wife, Judy, for her encouragement and her editorial assistance and typing. 